\title{
ON HOMOLOGICAL SITUATION PROPERTIES OF COMPLEXES AND CLOSED SETS
}

\author{
BY \\ PAUL ALEXANDROFF
}

The purpose of this paper is to find and to study topological invariants which connect the homological properties of a space $K$ with those of its closed subset $A$ and of the open complement $G=K \backslash A$, and thus help to characterize from the homological point of view the situation of $A$ in $K$.

In the case when $K$ is simply connected (that is when the Betti groups of $K$ are zero) the problem is solved by the duality theorems of Alexander, Pontrjagin, and Kolmogoroff, which determine the Betti groups of $G$ through the Betti groups of $A$. In the other special case when $K$ is a manifold the first duality theorems have been obtained by Pontrjagin [11](1) and Lefschetz [9] in 1927-1928. All these results are special cases of the general theory to which the present paper is devoted and which gives the very elementary construction (of the so-called extension- and intersection-homomorphisms, section 11) dominating the whole variety of duality and other situation properties.

The complete combinatorial theory is given in Chapter I for an arbitrary cell complex $K$ and its closed subcomplex $A$. In Chapter III the same theory is generalized for locally bicompact normal spaces $K$ and their closed sets $A$; this generalization is based on an approximation process developed in Chapter II. Chapter IV deals with manifolds and gives an elementary proof (combinatorial in character) of the Alexander-Pontrjagin duality in its most general form.

All main results obtained are completely formulated in the first four sections of Chapter I (sections 11-14). Numerical consequences are given in section 16. Section 18 deals with the Phragmén-Brouwer problem, while in section 19 some quite elementary examples are given as illustration.

The elementary known facts and notations used throughout this paper are systematized in the Introduction; its first part contains the group theoretical material, the second the needed information on complexes. Thus the present paper is practically independent of the previous literature on related subjects. There are only few references to my paper [1], and each of them may be read without reading the rest of that paper.

\section{INTRODUCTION}

\section{\$1. GROUPS AND THEIR CHARACTER GROUPS}

1. Groups, homomorphisms, duality. All groups considered are commuta-

Presented to the Society, April 24, 1943; received by the editors February 17, 1943.

(1) Numbers in brackets refer to the bibliography at the end of the paper. 
tive and written additively. In topological groups only closed subgroups and continuous homomorphisms are allowed. The difference- (or factor-) group of a group $X$ over its subgroup $X_{0}$ is denoted by $X-X_{0}$.

Let $\sigma$ be a homomorphism of the group $X$ into the group $Y$; let $Y_{1}=\sigma X$ be the image of $X$ under $\sigma$ and $X_{0}=\sigma^{-1} 0$ (or $\sigma^{-1} 0_{Y}$ ) be the kernel of the homomorphism $\sigma$, that is, the subgroup of all elements of $X$ mapped by $\sigma$ on the zero element $0_{Y}$ of $Y$. Then we of ten use the picture

$$
\frac{X \supseteq X_{0}}{Y \supseteq Y_{1}^{\downarrow}} .
$$

We denote by $I$ the additive group of all integers, by $\bar{I}$ the additive continuous (bicompact) group of all real numbers reduced modulo 1 .

If $B$ is the character group of the group $A$ in the sense of Pontrjagin [10] then $A$ is the character group of $B$, and we write

$$
A \mid B \text {. }
$$

The groups $A$ and $B$ are called in this case $d u a l$ and the relation (1.1) is called a duality.

2. Scalar products. If $A \mid B, a \in A, b \in B$, then the character $b$ of the group $A$ and the character $a$ of the group $B$ map respectively the elements $a \in A$ and $b \in B$ on the same element

$$
b a=a b \in \bar{I}
$$

called the product of $a$ and $b$.

Other cases in which products are defined are:

1. When $a$ and $b$ are elements of the same (commutative) ring.

2. When $a$ is an element of an arbitrary group $A$, while $b$ is an integer. Then for $b>0$

$$
\begin{aligned}
a b & =b a=a+a+\cdots+a \quad . \quad(b \text { times }), \\
a(-b) & =(-b) a=-(a b),
\end{aligned}
$$

and $a b=b a=0$ for $b=0$.

The scalar product

$$
(x \cdot y)=\sum a_{i} b_{i}
$$

of two linear forms $x=\sum a_{i} t_{i}$ and $y=\sum b_{i} t_{i}$ is defined if the products $a_{i} b_{i}$ are defined.

3. Annihilators. Let $X \mid Y$; let $A \subseteq X$ and $B \subseteq Y$ be subgroups of $X$ and $Y$ respectively. If $B$ is the annihilator of $A$ (in $Y$ ), that is, the subgroup of all elements $y$ of $Y$ satisfying the relation $x y=0$ whenever $x \in A$, then $A$ is the annihilator of $B$ in $X$ and we write

$$
X \supseteq A \perp B \subseteq Y,
$$


this relation being called an annihilation.

A fundamental theorem of the character theory is:

THEOREM 3. The annihilation $X \supseteq A \perp B \subseteq Y$ implies the dualities

$$
(X-A)|B, .(Y-B)| A .
$$

4. Conjugate homomorphisms. The fundamental lemma. Let

$$
X|\bar{X}, \quad Y| \bar{Y} \text {. }
$$

The homomorphisms

$$
\sigma \text { of } X \text { into } Y ; \quad \bar{\sigma} \text { of } \bar{Y} \text { into } \bar{X}
$$

are called conjugate if $(\sigma x \cdot \bar{y})=(x \cdot \bar{\sigma} \bar{y})$, whenever $x \in X, \bar{y} \in \bar{Y}$. In this case we write

$$
\sigma\left|\begin{array}{ll}
X & \bar{X} \uparrow \\
Y & \bar{Y}
\end{array}\right| \bar{\sigma}
$$

or-if the images $Y_{1}=\sigma X, \bar{X}_{1}=\bar{\sigma} \bar{Y}$ and the kernels $X_{0}=\sigma^{-1} 0_{Y}, \bar{Y}_{0}=\bar{\sigma}^{-1} 0_{\bar{X}}$ are given-

$$
\sigma\left|\begin{array}{ll}
\frac{X \supseteq X_{0}}{\bar{X}_{1} \subseteq \dot{\bar{X}}} \\
Y \supseteq Y_{1}^{\downarrow} & \frac{\bar{Y}_{0} \subseteq \bar{Y}}{\bar{Y}_{0}}
\end{array}\right| \bar{\sigma} .
$$

Most of this paper is based on the

Fundamental lemma 4. From (4.1) follows

$$
\left\{\begin{array}{l}
X \supseteq X_{0} \\
Y \supseteq Y_{1}^{\downarrow} / \perp \frac{\bar{X}_{1} \subseteq \bar{X}}{\bar{Y}_{0} \subseteq \bar{Y}}
\end{array}\right.
$$

(where the diagonal line means the duality $\bar{X}_{1} \mid Y_{1}$ ) and

$$
\begin{aligned}
X_{0} \mid\left(\bar{X}-\bar{X}_{1}\right) \\
\left(Y-Y_{1}\right) \mid \bar{Y}_{0} .
\end{aligned}
$$

The dualities (4.3) follow from Theorem 3 and the annihilations in (4.2), while the duality $\bar{X}_{1} \mid Y_{1}$ follows from Theorem 3 by means of the isomorphism $Y_{1} \approx X-X_{0}$. Thus we need to prove only the annihilations in (4.2). It is sufficient to prove the first annihilation

$$
X \supseteq X_{0} \perp \bar{X}_{1} \subseteq \bar{X}
$$

To this end let $x \in X_{0}, \bar{y} \in \bar{Y}$. Then

$$
(x \cdot \bar{\sigma} \bar{y})=(\sigma x \cdot \bar{y})=0 .
$$


On the other hand, if $\sigma x \neq 0$, then there exists a $\bar{y} \in \bar{Y}$ with

$$
(x \cdot \bar{\sigma} \bar{y})=(\sigma x \cdot \bar{y}) \neq 0 .
$$

Lemma 4 is proved.

5. Remark concerning the rational case. Let $\Re$ be the field of all rational numbers, $\Re^{n}$ the corresponding $n$-dimensional linear space, that is, the group of all linear forms with $n$ variables and rational coefficients; the group $\Re^{n}$ is considered as a group with operators, the latter being rational numbers. Thus only linear subspaces are allowed as subgroups. If we understand now by a character of $\Re^{n}$ a homomorphism of $\Re^{n}$ into $\Re$, the "character group" of $\Re^{n}$ is again $\Re^{n}$, as follows from the definition $y(x)=x(y)=(x \cdot y)$ for any $x \in \Re^{n}$, $y \in \Re^{n}$, the scalar product being understood in the most elementary sense. Thus the duality relation | turns in this case in to the identity and Lemma 4 still holds but now becomes trivial.

\section{§2. CONCERNING CELL COMPLEXES}

6. Cell spaces and their subspaces. A cell space (Tucker [14], Kolmogoroff [8]) is a finite set of elements, called cells, satisfying the following conditions:

1. To any cell corresponds a non-negative integer, the dimension of the cell (denoted by a superscript: $t^{r}$ is an $r$-dimensional cell or an $r$-cell).

2. To any cell $t^{r}$ corresponds a well defined cell $-t^{r}$ of the same dimension $r$, and we have $-\left(-t^{r}\right)=t^{r}$; the cells $t^{r}$ and $-t^{r}$ are called opposite (to each other).

3. To any two cells $t^{r}$ and $t^{r-1}$ of dimensions $r$ and $r-1$, respectively, corresponds an integer $\left(t^{r}: t^{r-1}\right)$, the incidence coefficient of $t^{r}$ and $t^{r-1}$, under the conditions:

$$
\left(-t^{r}: t^{r-1}\right)=\left(t^{r}:-t^{r-1}\right)=-\left(t^{r}: t^{r-1}\right) .
$$

It is convenient to suppose that in any pair of opposite cells of the cell space $K$ a definite cell is denoted by $t_{i}$ and the other by $-t_{i}^{*}$. Then we put

$$
\stackrel{r}{\epsilon}_{i j}=\left(\stackrel{r}{t_{i}: t_{i}^{r-1}}\right) \text {. }
$$

Let $t_{i}^{r} \in K$ and $t_{k}^{s} \in K$ be two cells, and $s<r$. We write $t_{i}^{r}>t_{k}^{s}$ or $t_{k}^{s}<t_{i}^{r}$ if cells $t_{j_{1}}^{r-1} \in K, \cdots, t_{j_{r-s-1}}^{s+1} \in K$ can be found so that

$$
\left(t_{i}^{r}: t_{j_{1}}^{r-1}\right) \neq 0,\left(t_{j_{1}}^{r-1}: t_{j_{2}}^{r-2}\right) \neq 0, \cdots,\left(t_{j_{r-s-1}+1}^{s+1}: t_{k}^{s}\right) \neq 0 .
$$

If $t_{k}^{s}<t_{i}^{\tau}$ we say that $t_{k}^{s}$ is a face of $t_{i}^{t}$.

A cell space $Q$ is called a subspace of the cell space $K$ if $Q$ is a subset of $K$ and

(a) two cells are opposite in $Q$ if and only if they are opposite in $K$; 
(b) the dimension of a cell and the incidence coefficient of two cells in $Q$ are the same as in $K$.

A subspace $Q$ of the cell space $K$ is called: closed, if $t \in Q, t^{\prime}<t$ (in $K$ ) implies $t^{\prime} \in Q$; open, if $t \in Q, t^{\prime}>t$ (in $K$ ) implies $t^{\prime} \in Q$.

7. Chains and boundaries. An r-dimensional chain (or an $r$-chain) on a cell space $K$ with respect to the group $X$ (the "coefficient-group") is a function $x^{r}$ with values in $X$ defined on the set of all $r$-dimensional cells of $K$, taking opposite values on opposite cells. The additive group of all $r$-dimensional chains on $K$ with respect to $X$ is denoted by $L_{K}^{r}(X)$ or by $L_{K}^{r}$. Chains with respect to the group $I$ of all integers are called integer chains.

The chain taking the value $a \in X$ on the cell $t^{\prime} \in K$ and the value zero on all cells different from $\pm t^{r}$ is denoted by $a t^{r}$ (in particular, the integer chain taking the value 1 on $t^{r}$, the value -1 on $-t^{r}$ and the value 0 elsewhere is identified with the cell $t^{r}$ ). This convention allows one to set for any chain $x^{r} \in L_{K}^{r}$

$$
x^{r}=\sum a_{i} t_{i}^{r}
$$

where $a_{i}$ is the value taken by $x^{r}$ on $t_{i}^{r}$.

The scalar product

$$
\left(x^{r} \cdot y^{r}\right)=\sum a_{i} b_{i}
$$

is defined for two chains $x^{r}=\sum a_{i} t_{i}^{r}$ and $y^{r}=\sum b_{i} t_{i}^{r}$ under the hypotheses for coefficient-groups mentioned in section 2 . In particular the scalar product $\left(x^{r} \cdot t_{t}^{r}\right)$ is always defined and equal to the value of the chain $x^{r}$ on the cell $t_{t}^{r}$.

Let $x^{r}$ be any chain of the cell space $K$. The $(r-1)$-chain taking the value $\sum_{i} \epsilon_{i j}^{\tau}\left(x^{r} \cdot t_{i}^{r}\right)$ on any $t_{j}^{r-1} \in K$ is denoted by $\Delta x^{r}$ and called the $\Delta$-boundary (or the lower boundary) of $x^{r}$ (on $K$ ). The $(r+1)$-chain taking the value $\sum_{i} \epsilon_{h t}^{r+1}\left(x^{r} \cdot t_{i}^{r}\right)$ on $t_{h}^{r+1} \in K$ is denoted by $\nabla x^{r}$ and called the $\nabla$ - (or upper) boundary of $x^{r}$ on $K$; sometimes we write $\Delta_{K} x^{r}, \nabla_{K} x^{r}$ instead of $\Delta x^{r}, \nabla x^{r}$ to show the cell space on which $\Delta, \nabla$ operate.

Obviously

$$
\left(\nabla t_{i}^{r} \cdot t_{h}^{r+1}\right)=\left(\Delta t_{h}^{r+1} \cdot t_{i}^{r}\right)=\stackrel{r+1}{\epsilon_{h i}},
$$

and thus for any two chains $x^{r}$ and $y^{r+1}$

$$
\left(\nabla x^{r} \cdot y^{r+1}\right)=\left(x^{r} \cdot \Delta y^{r+1}\right)
$$

provided that these scalar products have a sense (section 2).

Let $X \mid \bar{X}, L_{K}^{r}=L_{K}^{r}(X), \bar{L}_{K}^{r}=L_{K}^{r}(\bar{X})$. Denote by

$$
H_{K}^{r}=\nabla L_{K}^{r-1} ; \quad \bar{H}_{K}^{r}=\Delta \bar{L}_{K}^{r+1}
$$

the images; by $Z_{K}^{r}, \bar{Z}_{K}^{r}$ the kernels of the homomorphisms

$$
\nabla\left(\text { of } L_{K}^{r-1} \text { into } L_{K}^{r}\right) ; \quad \Delta\left(\text { of } \bar{L}_{K}^{r+1} \text { in to } \bar{L}_{K}^{r}\right. \text { ). }
$$


The elements of $Z_{K}^{r}$ (of $\bar{Z}_{K}^{r}$ ) are called $r$-dimensional $\nabla$-cycles ( $\Delta$-cycles); among all elements $x^{r}$ of $L_{K}^{r}$ (of $\bar{L}_{K}^{r}$ ) they are characterized by the condition

$$
\nabla x^{r}=0 ; \quad \Delta x^{r}=0 .
$$

From (7.1) and the Fundamental Lemma 4 follows

$$
\begin{aligned}
& \nabla\left|\begin{array}{l}
\frac{L_{K}^{r-1} \supseteq Z_{K}^{r-1}}{} \perp \bar{H}_{K}^{r-1} \subseteq \bar{L}_{K}^{r-1} \\
L_{K}^{r} \supseteq \bar{H}_{K}^{r} \perp
\end{array}\right| \Delta, \\
& \left(L_{K}^{r}-H_{K}^{r}\right) \mid \bar{Z}_{K}^{r} .
\end{aligned}
$$

8. Cell complexes. A cell space is called a cell complex if for each of its cells $\Delta \Delta t^{r}=0$, that is $\sum_{j} \epsilon_{i \epsilon_{j k}^{r}}^{r-1}=0$ for every $r, i, k$. On a cell complex $\Delta \Delta x^{r}=0$, $\nabla \nabla x^{r}=0$ for every chain $x^{r}$ and therefore $H_{K}^{r} \subseteq Z_{K}^{r}, \bar{H}_{K}^{r} \subseteq \bar{Z}_{K}^{r}$. The elements of $H_{K}^{r}, \bar{H}_{K}^{r}$ are called bounding cycles ( $\nabla$ - and $\Delta$-, respectively).

9. Betti groups. These are the groups

$$
\nabla_{K}^{r}=\nabla_{K}^{r}(X)=Z_{K}^{r}-H_{K}^{r} ; \quad \Delta_{K}^{r}=\Delta_{K}^{r}(\bar{X})=\bar{Z}_{K}^{r}-\bar{H}_{K}^{r} ;
$$

the first is called the $r$-dimensional $\nabla$ - (or upper) group and the second is called the $r$-dimensional $\Delta$ - (or lower) group of $K$ with respect to the given coefficient-group ( $X$ for $\nabla$ and $\bar{X}$ for $\Delta$ ). The elements of $\nabla_{K}^{r}$ (of $\Delta_{K}^{r}$ ) are called $r$-dimensional $\nabla$-classes ( $\Delta$-classes) of $K$. Two $\nabla$-cycles $\left(\Delta\right.$-cycles) $z_{1}^{r}$ and $z_{2}^{r}$ are called homologous to each other on $K$ if they belong to the same $\nabla$-class ( $\Delta$-class); we write in this case $z_{1}^{r} \sim z_{2}^{r}$ on $K$.

Let us prove the duality

$$
\nabla_{K}^{r} \mid \Delta_{K}^{r}
$$

We recall from the character theory that from $A|\bar{A}, C| \bar{C}, C=A-B$ follows $B \mid \bar{A}-\bar{C}$. Apply this to the Emmy Noether isomorphism

$$
\left(L_{K}^{r}-H_{K}^{r}\right)-\left(Z_{K}^{r}-H_{K}^{r}\right) \approx L_{K}^{r}-Z_{K}^{r}
$$

and take from (7.3), (7.2) (with $r+1$ instead of $r$ )

We get

$$
\left(L_{K}^{r}-H_{K}^{r}\right)\left|\bar{Z}_{K}^{r}, \quad L_{K}^{r}-Z_{K}^{r} \approx H_{K}^{r+1}\right| \bar{H}_{K}^{r} .
$$

q.e.d.

$$
\left(Z_{K}^{r}-H_{K}^{r}\right) \mid\left(\bar{Z}_{K}^{r}-\bar{H}_{K}^{r}\right),
$$

10. Closed and open subcomplexes. Let $Q$ be a closed subspace of the cell complex $K$ and $t^{r} \in Q$. Then $\Delta_{Q} t^{r}=\Delta_{K} t^{r}$ and $\Delta_{Q} \Delta_{Q} t^{r}=\Delta_{K} \Delta_{K} t^{r}=0$; thus $Q$ is a cell complex.

Let $Q$ be an open subspace of the cell complex $K$. Take any chain $x^{r}$ on $Q$ and define the chain $E_{K}^{Q} x^{r}$, called the trivial extension of $x^{r}$ over $K$, by setting 
$E_{K}^{Q} x^{r}=x^{r}$ on $Q, E_{K}^{Q} x^{r}=0$ on $K \backslash Q$. Then since $Q$ is open on $K$, we have for any $t^{r-1} \in Q$

$$
\left(\Delta_{Q} x^{r} \cdot t^{r-1}\right)=\left(\Delta_{K} E_{K}^{Q} x^{r} \cdot t^{r-1}\right)
$$

It follows that

$$
\left(\Delta_{Q} \Delta_{Q} x^{r} \cdot t^{r-2}\right)=\left(\Delta_{K} \Delta_{K} E_{K}^{Q} x^{r} \cdot t^{r-2}\right)=0
$$

for any $t^{r-2} \in Q$, and $Q$ is again a cell complex.

Thus closed as well as open subspaces of a cell complex are cell complexes.

Chapter I. Complexes

§3. THE GENERAL THEORY

11. The extension- and the intersection-homomorphisms. Let $Q$ be a subcomplex of the cell complex $K$ and $x_{Q}^{r}$ a chain on $Q$. Any chain on $K$ taking on the cells of $Q$ the same values as the chain $x_{Q}^{r}$ is called an extension of $Q$ (over $K$ ). Among these extensions the trivial extension $E_{K}^{Q} x_{Q}^{r}$ takes the value zero on $K \backslash Q$.

If we assign to any chain on $Q$ its trivial extension $E_{K}^{Q} x_{Q}^{r}$, we get the extension-isomorphism of $L_{Q}^{r}$ in to $L_{K}^{r}$.

REMARK. If there is no possibility of confusion, we shall identify chains with their trivial extensions and thus consider the extension-isomorphism as the identical isomorphism of $L_{Q}^{r}$ in to $L_{K}^{r} \supseteq L_{Q}^{r}$.

Now, let $x_{K}^{r}$ be any chain on $K$. We denote by $J_{Q}^{K} x_{K}^{\tau}$ the chain on $Q$ taking on the cells of $Q$ the same values as the chain $x_{K}^{r}$. If we assign to any chain $x^{r}$ on $K$ the chain $J_{Q}^{K} x_{K}^{r}$, we get a homomorphism $J_{Q}^{K}$ of $L_{K}^{r}$ in to $L_{Q}^{r}$ called the intersection-homomorphism.

Obviously, if $X \mid \bar{X}, L_{K}^{r}=L_{K}^{r}(X), \bar{L}_{K}^{r}=L_{K}^{r}(\bar{X})$, then

$$
J_{Q}^{K}\left|\begin{array}{cc}
L_{K}^{r} & \bar{L}_{K}^{r} \uparrow \\
L_{Q}^{r} & \bar{L}_{Q}^{r}
\end{array}\right| E_{K}^{Q} .
$$

From now to the end of this chapter $K$ is a fixed cell complex, $A$ is a fixed closed subcomplex of $K$ and $G=K \backslash A$ is the complementary open subcomplex.

It is easily seen that for any chains $x_{A}^{r}$ on $A, x_{G}^{\tau}$ on $G, x_{K}^{\tau}$ on $K$ :

$$
\begin{array}{ll}
\Delta E_{K}^{A} x_{A}^{r}=E_{K}^{A} \Delta x_{A}^{r} ; & \nabla E_{K}^{G} x_{G}^{r}=E_{K}^{G} \nabla x_{G}^{r}, \\
\nabla J_{A}^{K} x_{K}^{r}=J_{A}^{K} \nabla x_{K}^{r} ; & \Delta J_{G}^{K} x_{K}^{r}=J_{G}^{K} \Delta x_{K}^{r} .
\end{array}
$$

11.4. Therefore, the extension isomorphism maps identically

$$
\begin{array}{ll}
\Delta \text {-cycles of A } & \nabla \text {-cycles of } G \\
\text { on } \Delta \text {-cycles of } K & \text { on } \nabla \text {-cycles of } K
\end{array}
$$

while bounding cycles are mapped on bounding cycles. Thus the extension- 
isomorphism generates a homomorphism called the extension-homomorphism:

$$
E_{K}^{A} \text { of } \Delta_{A}^{r} \text { into } \Delta_{K}^{r} ; \quad E_{K}^{G} \text { of } \nabla_{G}^{r} \text { into } \nabla_{K}^{r} \text {. }
$$

11.5. The intersection-homomorphism maps $\nabla$-cycles of $K$ on $\nabla$-cycles of $A ; \Delta$-cycles of $K$ on $\Delta$-cycles of $G$ (while bounding cycles are mapped on bounding cycles) and thus generates a homomorphism, also called the intersection-homomorphism:

$$
J_{A}^{K} \text { of } \nabla_{K}^{r} \text { into } \nabla_{A}^{r} ; \quad J_{G}^{K} \text { of } \Delta_{K}^{r} \text { into } \Delta_{G}^{r} .
$$

12. The groups $\Delta_{K A}^{r}, \nabla_{K G}^{r} ; \Delta_{A: K}^{r}, \nabla_{G: K}^{r} ; \nabla_{A K}^{r}, \Delta_{G K}^{r}$. We write $0_{K}$ for the zero element of both $\Delta_{K}^{r}$ and $\nabla_{K}^{r}$, and use $0_{A}, 0_{G}$ in an analogous sense. Now we define

$$
\begin{aligned}
& \Delta_{K A}^{r}=E_{K}^{A} \Delta_{A}^{r} \subseteq \Delta_{K}^{r} ; \quad \nabla_{K G}^{r}=E_{K}^{G} \nabla_{G}^{r} \subseteq \nabla_{K}^{r} \quad \ldots \quad \text { (images), } \\
& \Delta_{A: K}^{r}=\left(E_{K}^{A}\right)^{-1} 0_{K} \subseteq \Delta_{A}^{r} ; \quad \nabla_{G: K}^{r}=\left(E_{K}^{G}\right)^{-1} 0_{K} \subseteq \nabla_{G}^{r} \quad \text { (kernels), } \\
& \nabla_{A K}^{r}=J_{A}^{K} \nabla_{K}^{r} \subseteq \nabla_{A}^{r} ; \quad \Delta_{G K}^{r}=J_{G}^{K} \Delta_{K}^{r} \subseteq \Delta_{G}^{r} \quad \text { (images), }
\end{aligned}
$$

and prove for the kernels of the intersection-homomorphism:

$$
\left(J_{A}^{K}\right)^{-1} 0_{A}=\nabla_{K G}^{r} ; \quad\left(J_{G}^{K}\right)^{-1} 0_{G}=\Delta_{K A}^{r} .
$$

We prove only the left-hand formula (writing $J$ for $J_{A}^{K}$ and $E$ for $E_{K}^{A}$ ).

1. If $z^{r} \in Z_{K}^{r}, z^{r}=z_{G}^{r}+h^{r}$ with $z_{G}^{r} \in Z_{G}^{r}, h^{r} \in H_{K}^{r}$, then $J z^{r}=J h^{r} \in H_{A}^{r}$.

2. If $z^{r} \in Z_{K}^{r}, J z^{r} \in H_{A}^{r}$, then $z^{r}=z_{G}^{r}+h^{r}$ with $z_{G}^{r} \in Z_{G}^{r}, h^{r} \in H_{K}^{r}$.

Let us prove 2. We have $J z^{r}=\Delta x_{A}^{r-1}, x_{A}^{r-1} \in L_{A}^{r-1}$. For any $t_{A}^{r} \in A$

Now

$$
\left(\left(z^{r}-\nabla E x_{A}^{r-1}\right) \cdot t_{A}^{r}\right)=\left(z^{r} \cdot t_{A}^{r}\right)-\left(\nabla E x_{A}^{r-1} \cdot t_{A}^{r}\right) .
$$

Hence

$$
\begin{gathered}
\left(z^{r} \cdot t_{A}^{r}\right)=\left(J z^{r} \cdot t_{A}^{r}\right)=\left(\nabla x_{A}^{r-1} \cdot t_{A}^{r}\right), \\
\left(\nabla E x_{A}^{r-1} \cdot t_{A}^{r}\right)=\left(J \nabla E x_{A}^{r-1} \cdot t_{A}^{r}\right)=\left(\nabla J E x_{A}^{r-1} \cdot t_{A}^{r}\right)=\left(\nabla x_{A}^{r-1} \cdot t_{A}^{r}\right) .
\end{gathered}
$$

$$
\left(\left(z^{r}-\nabla E x_{A}^{r-1}\right) \cdot t_{A}^{r}\right)=0 \quad \text { for } t_{A}^{r} \in A
$$

so that the cycle $z^{r}-\nabla E x_{A}^{r-1}$ is on $G$. As $\nabla E x_{A}^{r-1} \in H_{K}^{r}$ the assertion 2 and thus the formula (12.4) are proved.

13. The geometrical meaning of the groups $\Delta_{K A}^{r}, \nabla_{K G}^{r} ; \Delta_{A: K}^{r}, \nabla_{G: K}^{r} ; \nabla_{A K}^{r}, \Delta_{G K}^{r}$.

13.1. The subgroup

$$
\Delta_{K A}^{r} \text { of } \Delta_{K}^{r} ; \quad \nabla_{K G}^{r} \text { of } \nabla_{K}^{r}
$$

defined as the image of $\Delta_{A}^{r}$ (of $\nabla_{G}^{r}$ ) under the extension-homomorphism has for its elements those $\Delta$-classes ( $\nabla$-classes) of $K$ which contain $\Delta$-cycles on $A$ $(\nabla$-cycles on $G$ ). 
13.2. Take all those

$$
\Delta \text {-classes } \underset{z_{A}}{r} \in \Delta_{A}^{r} ; \quad \nabla \text {-classes }{ }_{z_{G}}^{r} \in \nabla_{a}^{r}
$$

whose elements bound on $K$. The resulting subgroup of $\Delta_{A}^{r}$ (of $\nabla_{G}^{r}$ ) is the group $\Delta_{A: K}^{r}$ (the group $\nabla_{G: K}^{r}$ ), defined as the kernel of the extension-homomorphism.

Definition 13.3. $A \nabla$-cycle on $A$ (a $\Delta$-cycle on $G$ ) is called extensible (over $K$ ) if among its extensions over $K$ there are $\nabla$-cycles. $(\Delta$-cycles) on $K$.

13.4. Then the group $\nabla_{A K}^{r}$ (the group $\Delta_{G K}^{r}$ ) defined as the image of $\nabla_{K}^{r}$ (of $\Delta_{K}^{r}$ ) under the intersection-homomorphism is the difference-group of the group of all $r$-dimensional extensible $\nabla$-cycles on $A$ ( $\Delta$-cycles on $G$ ) over the subgroup of cycles bounding on $A$ (on $G$ ).

14. The results. From the definition of the groups involved, from (11.1) and (12.4) follows by the Fundamental Lemma 4:

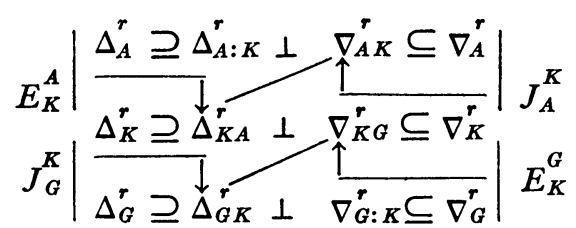

and

$$
\Delta_{A: K}^{r}\left|\left(\nabla_{A}^{r}-\nabla_{A K}^{r}\right) ; \quad \nabla_{G: K}^{r}\right|\left(\Delta_{G}^{r}-\Delta_{G K}^{r}\right) .
$$

The picture (14.1) contains

1. two pairs of isomorphisms: the first pair

$$
\Delta_{A}^{r}-\Delta_{A: K}^{r} \approx \Delta_{K A}^{r} ; \quad \nabla_{G}^{r}-\nabla_{G: K}^{r} \approx \nabla_{K G}^{r} ;
$$

the second pair

$$
\Delta_{K}^{r}-\Delta_{K A}^{r} \approx \Delta_{G K}^{r} ; \quad \nabla_{K}^{r}-\nabla_{K G}^{r} \approx \nabla_{A K}^{r},
$$

2. the pair of dualities

$$
\Delta_{K A}^{r}\left|\nabla_{A K}^{r} ; \quad \nabla_{K G}^{r}\right| \Delta_{G K}^{r}
$$

3. three annihilations: the central annihilation

$$
\Delta_{K}^{r} \supseteq \Delta_{K A}^{r} \perp \nabla_{K G}^{r} \subseteq \nabla_{K}^{r}
$$

and the pair of annihilations

$$
\Delta_{A}^{r} \supseteq \Delta_{A: K}^{r} \perp \nabla_{A K}^{r} \subseteq \nabla_{A}^{r} ; \quad \nabla_{G}^{r} \supseteq \nabla_{G: K}^{r} \perp \Delta_{G K}^{r} \subseteq \Delta_{G}^{r} .
$$

In each pair of relations (isomorphisms, dualities, annihilations) the one relation is obtained from the other by interchanging $\Delta$ and $\nabla, A$ and $G$. 
From these relations the first pair of isomorphisms is a triviality, being contained in the very definition of the groups involved.

The dualities in (14.1) and (14.2) are algebraic consequences of the annihilations and the latter follow from the fact that the extension- and intersection-homomorphisms are conjugate.

The central annihilation may be brought obviously into the form of the following

REMOVING THEOREM. In order that an $r$-dimensional $\nabla$-cycle $(\Delta$-cycle $)$ on $K$ be homologous on $K$ to a

$$
\nabla \text {-cycle on } G, \quad \Delta \text {-cycle on } A
$$

it is necessary and sufficient that its scalar product with each $r$-dimensional $\Delta$-cycle on $A ; r$-dimensional $\nabla$-cycle on $G$ be equal to zero $\left(^{2}\right)$.

In addition to (14.1) and (14.2) we have

The MAIN DUALITY THEOREM.

$$
\Delta_{A: K}^{r} \mid \nabla_{G: K}^{r+1} .
$$

We give to this fundamental duality the form of a pair of isomorphisms by means of the following definitions:

$$
\nabla_{A: K}^{r}=\nabla_{A}^{r}-\nabla_{A K}^{r} ; \quad \Delta_{G: K}^{r}=\Delta_{G}^{r}-\Delta_{G K}^{r} .
$$

REMARK. The groups $\nabla_{A: K}^{r}, \Delta_{G: K}^{r}$ can also be defined as the difference groups of the groups of all $r$-dimensional $\nabla$-cycles of $A$ ( $\Delta$-cycles of $G$ ) over the subgroups of all extensible cycles.

With these definitions we deduce from (14.2) the dualities

$$
\Delta_{A: K}^{r}\left|\nabla_{A: K}^{r} ; \quad \nabla_{G: K}^{r}\right| \Delta_{G: K}^{r}
$$

and transform the duality (14.3) into the third pair of isomorphisms:

$$
\Delta_{A: K}^{r} \approx \Delta_{G: K}^{r+1} ; \quad \nabla_{A: K}^{r} \approx \nabla_{G: K}^{r+1} .
$$

The groups $\Delta_{K A}^{r}, \nabla_{K G}^{r} ; \Delta_{A: K}^{r}, \nabla_{G: K}^{r} ; \nabla_{A K}^{r}, \Delta_{K G}^{r} ; \nabla_{A: K}^{r}, \Delta_{G: K}^{r}$ are called the groups of the figure $K, A, G$. It follows from (14.1)-(14.3) that they are all completely determined if we know for instance the groups $\Delta_{K}^{r}, \Delta_{A}^{r}, \Delta_{K A}^{r}$ (for all $r$ ).

In Chapters II and III the definitions of the extension- and of the intersection-homomorphisms as well as the definitions of the groups of the figure $K, A, G$ are generalized to the case of an arbitrary locally bicompact normal space $K$ and its subsets: the closed set $A \subseteq K$ and its open complement $G=K \backslash A$. We show in these chapters that all relations (14.1)-(14.3') hold in that general set theoretical case.

(2) I am indebted to L. Pontrjagin who first formulated the Removing Theorem and many times pointed out to me (as did also L. Lusternik) the desirability of proving it under the most general conditions. 
In particular, the duality theorem (14.3) (or the equivalent pair of isomorphisms $\left(14.3^{\prime}\right)$ ) is the definitive generalization of the duality theorem of Alexander-Kolmogoroff: If the space $K$ is simply connected in the dimension $r$ (that is, if one and therefore both of the equivalent conditions $\Delta_{K}^{r}=0$, $\nabla_{K}^{r}=0$ hold), then obviously $\Delta_{A: K}^{r}=\Delta_{A}^{r}, \nabla_{G: K}^{r}=\nabla_{G}^{r}$. Hence, if $K$ is simply connected in the dimensions $r$ and $r+1$ then (14.3') turns into the AlexanderKolmogoroff isomorphisms

$$
\Delta_{A}^{r} \approx \Delta_{G}^{r+1}, \quad \nabla_{A}^{r} \approx \nabla_{G}^{r+1} .
$$

Let us return to the combinatorial case, that is, let $K$ be a cell complex, $A$ a closed subcomplex of $K$. Taking as coefficient-group the group $I_{m}$ of all integers reduced modulo $m$ and denoting by $\Delta_{K}^{r}(m), \Delta_{A}^{r}(m), \Delta_{A: K}^{r}(m), \nabla_{K G}^{r}(m)$ and so on the corresponding groups (of $K$, of $A$, of $G$, of $K, A, G$ ) we see by the first pair of isomorphisms that the order of the group $\Delta_{A}^{r}(m)$ is equal to the product of the orders of $\Delta_{K A}^{r}(m)$ and $\Delta_{A: K}^{r}(m)$ and thus is completely determined by the last two groups. Now by a theorem of M. Bockstein [6, p. 373] all Betti groups of a complex $K$ are completely determined by the orders of the groups $\Delta_{K}^{r}(m)$ taken for all $m$ and all $r$. Thus the groups $\Delta_{A}^{r}$ are determined by the groups $\Delta_{K A}^{r}(m)$ and $\Delta_{A: K}^{r}(m)$ (taken for all $m$ and all $r$ ). An analogous result holds for $\nabla_{G}^{r}$, while $\Delta_{K}^{r}$ is determined by the $\Delta_{K A}^{r}(m)$ and $\nabla_{K G}^{r}(m)$.

14.4. Hence if $K$ is a cell complex, $A$ a closed subcomplex of $K$ and $G=K \backslash A$, then the groups $\Delta_{A}^{r}$ and $\Delta_{G}^{r}$ (and all groups of the figure $K, A, G$ ) for any $r$ and any coefficient-group are completely determined by the groups $\Delta_{K}^{r}(m), \Delta_{K A}^{r}(m)$ and $\Delta_{A: K}^{r}(m)$ taken for all $m$ and all $r$ (while the groups $\Delta_{K}^{r}$ are determined by $\Delta_{K A}^{r}(m)$ and $\nabla_{K G}^{r}(m)$ taken also for all $m$ and all $\left.r\right)$.

In Chapter III, section 40, we prove that Theorem 14.4 still holds if we understand by $K$ and $A$ topological images of (finite) polyhedrons (in the terminology of $[4, \mathrm{p} .128])$. Thus for topological images of finite polyhedrons the homological situation-problem may be considered as being completely solved.

REMARK. The third pair of isomorphisms is given for the first time in my notes [2].

15. The operator $\nabla E_{K}^{A}$ and the proof of the third pair of isomorphisms. To any $\nabla$-cycle $z_{A}^{r}$ on $A$ corresponds the $\nabla$-cycle $\nabla E_{K}^{A} z_{A}^{r}$ on $G$ which by. its very definition bounds on $K$. Let us prove: If $z_{A}^{r}$ is extensible, then $\nabla E_{K}^{A} z_{A}^{r}$ bounds on $G$. By supposition there exists a $\nabla$-cycle $z^{r}$ on $K$ such that $z_{A}^{r}=J_{A}^{K} z^{r}$ and therefore (we write $E$ instead of $E_{K}^{A}$ )

$$
\begin{aligned}
z^{r} & =E z_{A}^{r}+J_{G}^{K} z^{r}, \\
0=\nabla z^{r} & =\nabla E z_{A}^{r}+\nabla J_{G}^{K} z^{r}, \\
\nabla E z_{A}^{r} & =-\nabla J_{G}^{K} z^{r} .
\end{aligned}
$$


As $J_{G}^{K} z^{r}$ is a chain on $G, \nabla E z_{A}^{r}$ bounds on $G$ and our assertion is proved.

Thus the operator $\nabla E$ produces a homomorphism of $\nabla_{A: K}^{r}$ into $\nabla_{G: K}^{r+1}$. We call this homomorphism again $\nabla E$ and prove that it is an isomorphism of $\nabla_{A: K}^{r}$ on to $\nabla_{G: K}^{r+1}$.

To prove that $\nabla E$ maps $\nabla_{A: K}^{r}$ on the whole $\nabla_{G: K}^{r+1}$ take arbitrarily

$$
z_{G}^{r+1} \in{ }_{z_{G}^{r+1}}^{r+1} \in \nabla_{G: K}^{r+1}
$$

We have to find such a $\nabla$-cycle $z_{A}^{r}$ on $A$ that $z_{G}^{+1}-\nabla E z_{A}^{r}$ bounds on $G$.

By supposition there exists a chain $x^{r}$ on $K$ bounded by $z_{G}^{+1}$ and consequently

$$
\nabla_{A} J_{A}^{K} x^{r}=J_{A}^{K} \nabla x^{r}=J_{A}^{K} z_{G}^{r+1}=0 ;
$$

hence $z_{A}^{r}=J_{A}^{K} x^{r}$ is a $\nabla$-cycle on $A$ and $x_{G}^{r}=x^{r}-E J_{A}^{K} x^{r}$ is a chain on $G$ with

$$
\nabla x_{G}^{r}=\nabla x^{r}-\nabla E J_{A}^{K} x^{r}=z_{G}^{r+1}-\nabla E z_{A}^{r} .
$$

It remains to be proved that $\nabla E$ maps $\nabla_{A: K}^{r}$ on $\nabla_{G: K}^{r+1}$ isomorphically. To this end we show that if for a certain

$$
z_{A}^{r} \in{ }_{z_{A}}^{r} \in \nabla_{A}^{r}: K
$$

the $\nabla$-cycle $\nabla E z_{A}^{r}$ bounds on $G$ then there exists such a $\nabla$-cycle $z^{r}$ on $K$ that $z_{A}^{r}=J_{A}^{K} z^{r}$.

By supposition

$$
\nabla E z_{A}^{r}=\nabla \stackrel{r}{x_{G}}, \quad \stackrel{r}{x}_{G} \in L_{G}^{r}
$$

Therefore

$$
\nabla\left(E z_{A}^{r}-x_{G}^{r}\right)=0,
$$

so that $z^{r}=E z_{A}^{r}-x_{G}^{r}$ is a $\nabla$-cycle on $K$ with

$$
J_{A}^{K} z^{r}=J_{A}^{K} E z_{A}^{r}-J_{A}^{K} x_{G}^{r}=z_{A}^{r},
$$

q.e.d.

REMARK. In an analogous way to any $\Delta$-cycle $z_{G}^{+1}$ on $G$ corresponds the $\Delta$-cycle $\Delta E_{K}^{G} z_{G}^{r+1}$ on $A$ bounding on $K$, and the operator $\Delta E_{K}^{G}$ produces an isomorphism of the group $\Delta_{G: K}^{r+1}$ on to the group $\Delta_{A: K}^{r}$.

\section{§4. Special cases}

16. Numerical relations. In this section the coefficient-group is either the field $I_{m}, m$ being a prime, or the field $\Re$ of the rational numbers, the latter being treated according to the remark of section 5 . Now all dualities turn into isomorphisms.

Let $\rho$ denote the rank of a group of linear forms with respect to the given coefficient-field. We put $\pi_{K}^{r}=\rho \Delta_{K}^{r}=\rho \nabla_{K}^{r}$ (Betti numbers) and 


$$
\begin{aligned}
\pi_{K A}^{r}=\rho \nabla_{A K}^{r}=\rho \Delta_{K A}^{r} ; & \pi_{K G}^{r}=\rho \Delta_{G K}^{r}=\rho \nabla_{K G}^{r}, \\
\pi_{A: K}^{r}=\rho \Delta_{A: K}^{r}=\rho \nabla_{A: K}^{r} ; & \pi_{G: K}^{r}=\rho \nabla_{G: K}^{r}=\rho \Delta_{G: K}^{r} .
\end{aligned}
$$

Then by the first pair of isomorphisms

$$
\stackrel{r}{\pi_{A}}=\stackrel{r}{\pi_{A: K}}+{\stackrel{r}{\pi_{K A}} ;}_{r}^{r} \quad \pi_{G}^{r}=\pi_{G: K}^{r}+\pi_{K G}^{r},
$$

while by the second pair of isomorphisms

$$
\stackrel{r}{\pi_{K}}=\stackrel{r}{\pi_{K A}}+\stackrel{r}{\pi_{K G}}
$$

or

$$
\pi_{K}^{r}=\left(\pi_{A}^{r}-\pi_{A: K}^{r}\right)+\left(\pi_{G}^{r}-\pi_{G: K}^{r}\right) .
$$

Using the third pair of isomorphisms and changing $r$ into $r+1$ we get

$$
\stackrel{r+1}{\pi_{G}}=\stackrel{r}{\pi_{A}: K}+\pi_{K}^{r+1}-\left(\pi_{A}^{r+1}-\pi_{A: K}^{r+1}\right),
$$

or

$$
\pi_{G}^{r+1}=\pi_{A: K}^{r}+\left(\pi_{K}^{r+1}-\pi_{K A}^{r+1}\right) .
$$

In the special case when $K$ is an $n$-dimensional (orientable) manifold an equivalent formula has been given by Pontrjagin; in this case by the Poincaré duality (see section 42 below) we have $\pi_{G}^{r+1}=\pi_{G}^{n-r-1}$ and (16.4) can be written as

$$
\pi_{G}^{n-r-1}=\pi_{A: K}^{r}+\left(\pi_{K}^{r+1}-\pi_{K A}^{r+1}\right),
$$

which is the Pontrjagin formula [11, p. 449, Theorem II].

17. Application to regular components. Now suppose that $K$ is a homogeneously $n$-dimensional cell complex (that is, there are in $K$ no cells of a dimension greater than $n$ while each cell of a dimension less than $n$ is a face of an $n$-dimensional cell). An $(n-1)$-cell $t^{n-1}$ is called regular if there are in $K$ two cells $t_{1}^{n}$ and $t_{2}^{n}$ such that $t^{n-1}$ is a face of $\pm t_{1}^{n}$ and $\pm t_{2}^{n}$ and of no more cells of $K$. A (finite) sequence $t_{1}^{n}, \cdots, t_{s}^{n}$ of $n$-cells is called an $r$-sequence if in it any two subsequent cells have in $K$ a common face of a dimension at least $r-1$. Two $n$-cells of $K$ belong (with all their faces) to the same $r$-component of $K$ if they can be connected (in $K$ ) by an $r$-sequence. The cells common to two different $r$-components of $K$ are of dimension at most $r-2$. A complex is called $r$-connected if it consists only of one $r$-component; an $n$-connected complex $K$ is called an $n$-dimensional pseudomanifold if all of its $(n-1)$-cells are regular. There are orientable and non-orientable $n$-dimensional pseudomanifolds: the group $\nabla_{K}^{n}(I)$ of an $n$-dimensional pseudomanifold $K$ is always cyclic, infinite if $K$ is orientable, and of order 2 if $K$ is non-orientable. It follows that if the $n$-dimensional cell complex $K$ is in any way decomposed into a certain number of $n$-dimensional pseudomanifolds no two of which 
have a cell of dimension at least $n-1$ in common, then the group $\nabla_{K}^{n}(I)$ is the direct sum of as many infinite cyclic groups as there are orientable pseudomanifolds and of as many groups of order 2 as there are non-orientable pseudomanifolds in our decomposition.

Let $K$ be again an arbitrary homogeneously $n$-dimensional complex; denote by $A_{n-1}$ the subcomplex of $K$ consisting of all $(n-1)$-dimensional irregular cells and of all faces of these cells. The $n$-components of the open subcomplex $G_{n-1}=K \backslash A_{n-1}$ are called regular components of $K$ (we slightly change here the terminology of [4, p. 190]). The decomposition of $G_{n-1}$ in to its $n$-components (the regular components of $K$ ) is a decomposition of the kind just mentioned and therefore the number $q(K)$ of the regular components of $K$ is equal to the n-dimensional Betti number modulo 2 of $G_{n-1}$, while the number $q_{\circ}(K)$ of the orientable regular components of $K$ is the ordinary $n$-dimensional Betti number of $G_{n-1}$. Denoting as usual by $\pi^{r}$ the ordinary Betti numbers and by $\pi^{r}(2)$ the Betti numbers modulo 2 we get, using (16.4):

17.1. For any homogeneously $n$-dimensional complex $K$ :

$$
q(K)=\stackrel{n-1}{\pi_{A_{n-1}: K}(2)}+\pi_{K}^{n}(2) ; \quad q_{o}(K)=\stackrel{n-1}{\pi_{A_{n-1}}: K}+\stackrel{r}{\pi_{K}} .
$$

In particular, if all $(n-1)$-cells of $K$ are regular, then

$$
q(K)=\pi_{K}^{n}(2) ; \quad q_{o}(K)=\pi_{K}^{n} .
$$

This is the case with the complex $G=K \backslash A$ if the closed subcomplex $A \subset K$ contains all irregular $(n-1)$-cells of $K$. Therefore, applying again (16.4), we get:

17.2. If the closed subcomplex $A$ of the homogeneously $n$-dimensional complex $K$ contains all irregular $(n-1)$-cells of $K$, then

$$
q(G)=\pi_{A: K}^{n-1}(2)+\pi_{K}^{n}(2)-\pi_{K A}^{n}(2) ; \quad q_{o}(G)=\pi_{A: K}^{n-1}+\pi_{K}^{n}-\pi_{K A}^{n} .
$$

If $A$ is moreover ( $n-1)$-dimensional, then

$$
q(G)=\pi_{A: K}^{n-1}(2)+\pi_{K}^{n}(2) ; \quad q_{o}(G)=\pi_{A: K}^{n-1}+\pi_{K}^{n} .
$$

Now we call any cell of $K$ regular if the $n$-dimensional $\nabla^{n}$-group of its open star (with respect to the group $I$ ) is infinite cyclic. This definition agrees for $(n-1)$-cells with the previous one. If $A$ contains all irregular cells of dimensions at least $r$ then the $r$-components of $G$ are identical with the regular components of $G$. Thus for $r=0$, that is if $A$ contains all irregular cells of $K$, the ordinary components of $G$ coincide with the regular ones and their number is still given by (17.2) and, in the case of an ( $n-1)$-dimensional $A$, by (17.3).

REMARK. If no special hypotheses on the closed subcomplex $A$ are made, then the number of components of the open subcomplex $G=K \backslash A$ depends upon the homological properties of the mutual situation in $K$ of two subcomplexes: the given subcomplex $A$ and the subcomplex $A_{0}$ of all irregular ele- 
ments of $K$. Even the most elementary examples show that the features of this mutual situation may be very different.

18. The Phragmén-Brouwer theorem. Let $A$ be as always a closed subcomplex of the cell complex $K, G=K \backslash A$ and

$$
\begin{gathered}
A=A_{1} \cup A_{2}, \quad A_{1} \text { and } A_{2} \text { closed in } A, \\
Q_{1}=A \backslash A_{1}, \quad Q_{2}=A \backslash A_{2} ; \quad G_{1}=K \backslash A_{1}, \quad G_{2}=K \backslash A_{2}, \\
A_{0}=A_{1} \cap A_{2} .
\end{gathered}
$$

Denote finally by $E_{A: K}^{i}, i=1,2$, the homomorphism of $\nabla_{Q_{i}}^{r}$ into $\nabla_{A: K}^{r}$ which assigns to each element $z^{r}$ of $\nabla_{Q_{i}}^{r}$ the class $z^{r} \in \nabla_{A: K}^{r}$ containing the element $E_{A}^{Q_{i} z^{r}}$. We shall prove the isomorphism

$$
\left(E_{A: K}^{1} \nabla_{Q_{1}}^{r}\right) \cap\left(E_{A: K}^{2} \nabla_{Q_{2}}^{r}\right) \approx \nabla_{G: G_{1}}^{r+1} \cap \nabla_{G: G_{2}}^{r+1} .
$$

Note that the elements of the group $\left(E_{A: K}^{1} \nabla_{Q_{1}}^{r}\right) \cap\left(E_{A: K}^{2} \nabla_{Q_{2}}^{r}\right)$ can be defined as being those classes $z^{r} \in \nabla_{A: K}^{r}$ of $\nabla$-cycles which contain both cycles on $Q_{1}$ and $Q_{2}$; the elements of $\nabla_{G: G_{1}}^{r} \cap \nabla_{G: G_{2}}^{r}$ are $\nabla$-classes of $G$ whose elements bound both on $G_{1}$ and $G_{2}$.

The isomorphism (18.1) is realized by the operator $\nabla E_{K}^{A}$ : let $z_{0}^{r} \in\left(E_{A: K}^{1} \nabla_{Q_{1}}^{r}\right)$ $\cap\left(E_{A: K}^{2} \nabla_{Q_{2}}^{r}\right)$. Take $z_{0}^{r} \in z_{0}^{r}$ on $Q_{1}$. Then $E_{K}^{A} z_{0}^{r}$ is equal to zero on $A_{1}$, thus $\nabla E_{K}^{A} z_{0}^{r}$ bounds on $G_{1}$. Consequently all elements of the $\nabla$-class $\nabla E_{K \gamma_{0}{ }^{r}}^{A} \in \nabla_{G}^{r+1}{ }^{\circ}$ bound on $G_{1}$. Taking $z_{0}^{r} \in{ }_{z_{0}^{r}}^{r}$ on $Q_{2}$ we see in the same way that all elements of $\nabla E_{K z_{0}^{r}}^{A}$ bound on $G_{2}$. Hence the isomorphism $\nabla E_{K}^{A}$ maps the left-hand member of (18.1) into the right-hand one. To show that this mapping is on the righthand member of (18.1) let

$$
z_{G}^{r+1} \in E_{K z_{0}=}^{A r}={ }_{z G}^{r+1} \in \nabla_{G: G_{1}}^{r+1} \cap \nabla_{G: G_{2}}^{r+1} .
$$

As $z_{G}^{++1}$ bounds on $G_{i}, i=1,2$, it is $z_{G}^{+1}=\nabla x_{i}^{r}$ with $x_{i}^{r}$ on $G_{i}$. Therefore $z_{A}^{r}=J_{A}^{K} x_{i}^{r}$ is a $\nabla$-cycle on $Q_{i}$ and by (15.1) (with $x_{G}^{r}=x_{i}^{r}-E J_{A}^{K} x_{i}^{r}$ ) we have:

$$
\nabla E_{K z_{A}^{A}}^{A} \in z^{r+1},
$$

thus $z_{A}^{r} \in_{z_{0}^{r}}^{r}$, and ${ }_{z_{0}^{r}}^{r}$ contains cycles on $Q_{1}$ as well as cycles on $Q_{2}$, q.e.d.

Let $K$ be simply connected with respect to $r$; then the isomorphism (18.1) is replaced by

$$
\nabla_{A Q_{1}}^{r} \cap \nabla_{A Q_{2}}^{r} \approx \nabla_{G: G_{1}}^{r+1} \cap \nabla_{G: G_{2}}^{r+1}
$$

Now

therefore

$$
\Delta_{A}^{r} \supseteq \Delta_{A A_{i}}^{r} \perp \nabla_{A Q_{i}}^{r} \subseteq \nabla_{A}^{r}, \quad i=1,2,
$$

$$
\Delta_{A}^{r} \supseteq\left(\Delta_{A A_{1}}^{r}+\Delta_{A A_{2}}^{r}\right) \perp\left(\nabla_{A Q_{1}}^{r} \cap \nabla_{A Q_{2}}^{r}\right) \subseteq \nabla_{A}^{r}
$$

(the sum at left may fail to be direct) and 


$$
\left(\Delta_{A}^{r}-\left(\Delta_{A A_{1}}^{r}+\Delta_{A A_{2}}^{r}\right)\right) \mid\left(\nabla_{A Q_{1}}^{r} \cap \nabla_{A Q_{2}}^{r}\right) .
$$

To any $\Delta$-cycle $z^{r}$ on $A$ corresponds the $\Delta$-cycle

$$
\Delta J_{Q_{1} z^{r}}^{A}=-\Delta J_{A_{1} z^{r}}^{A} \text { on } A_{0}=A_{1} \cap A_{2}
$$

(where $\Delta$ operates on $A$ ). This correspondence generates a homomorphism (the "Naht-Homomorphismus" of [4, p. 292]) of $\Delta_{A}^{r}$ onto $\Delta_{A_{0}: A_{1}}^{r-1} \cap \Delta_{A_{0}: A_{2}}^{r-1}$ with the kernel $\Delta_{A A_{1}}^{r}+\Delta_{A A_{2}}^{r}$ (the proof is easy and is given in [4, pp. 289-293]). Thus

$$
\left(\Delta_{A}^{r}-\left(\Delta_{A A_{1}}^{r}+\Delta_{A A_{2}}^{r}\right)\right) \approx \Delta_{A_{0}: A_{1}}^{r-1} \cap \Delta_{A_{0}: A_{2}}^{r-1} .
$$

From (18.2)-(18.4) follows a duality which we call

The Phragmén-Brouwer theorem.

$$
\left(\Delta_{A_{0}: A_{1}}^{r-1} \cap \Delta_{A_{0}: A_{2}}^{r-1}\right) \mid\left(\nabla_{G: G_{1}}^{r+1} \cap \nabla_{G: G_{2}}^{r+1}\right) .
$$

\section{Examples.}

1. The elements of the complex $K$ are: a triangle, its edges and its vertices, thus the cells $\pm t^{2}, \pm t_{i}^{1}, \pm t_{i}^{0}, i=1,2,3$, with obvious incidence coefficients. The open subcomplex $G$ consists only of $\pm t^{2}$, the closed subcomplex $A$ of the remaining elements of $K$. The operator $\nabla E_{K}^{A}$ assigns to the $\nabla$-class $z_{A}^{1} \in \nabla_{A}^{1}$ consisting of the $\nabla$-cycles $t_{1}^{1}, t_{2}^{2}, t_{3}^{1}$ on $A$ the $\nabla$-class $z_{G}^{2} \in \nabla_{G}^{2}$ the only element of which is $t^{2}$. The groups $\nabla_{A}^{1}(I)$ and $\nabla_{G}^{2}(I)$ are both infinite cyclic (duality theorem of Alexander-Kolmogoroff).

2. Consider the circular ring in the plane decomposed into the cell complex $K$ whose elements are: $\pm t^{2}, \pm t_{i}^{1}, \pm t_{i}^{0}, i=1,2,3$, with the incidence coefficients

$$
\begin{gathered}
\left(t^{2}: t_{1}^{1}\right)=\left(t^{2}: t_{2}^{1}\right)=1, \quad\left(t^{2}: t_{3}^{1}\right)=0, \quad\left(t_{3}^{1}: t_{1}^{0}\right)=1, \quad\left(t^{1}: t_{2}^{0}\right)=-1, \\
\left(t_{i}^{1}: t_{j}^{0}\right)=0 \quad \text { if } \quad i, j=1,2 .
\end{gathered}
$$

The open subcomplex $G$ contains only the cells $\pm t^{2}$ and $A=K \backslash G$. All groups of the figure $K, A, G$ are determined by their ranks:

$$
\begin{aligned}
& {\stackrel{0}{\pi_{K}}}^{0}=\stackrel{\pi}{\pi}_{K A}^{0}=1 ; \quad \pi_{A}^{0}=2 ; \quad \stackrel{0}{\pi_{A: K}}=1 ; \quad \stackrel{0}{\pi_{G}}=\stackrel{0}{\pi_{K G}}=\stackrel{0}{\pi_{G: K}}=0, \\
& \stackrel{1}{\pi_{K}}=\stackrel{1}{\pi_{K A}}=1 ; \quad \stackrel{1}{\pi_{A}}=2 ; \quad \stackrel{1}{\pi_{A}: K}=1 ; \quad \stackrel{1}{\pi_{G}}=\stackrel{1}{\pi_{G}: K}=1 ; \quad \stackrel{1}{\pi_{K G}}=0, \\
& \stackrel{2}{\pi_{K}}=\stackrel{2}{\pi_{K A}}=\stackrel{2}{\pi_{A}}=\stackrel{2}{\pi_{A}: K}=\stackrel{2}{\pi_{K G}}=0 ; \quad \stackrel{2}{\pi_{G}}=\stackrel{2}{\pi_{G: K}}=1 .
\end{aligned}
$$

3. The elements of $K$ are $\pm t^{2}, \pm t^{1}$, $\pm t^{0}$ with the incidence coefficients $\left(t^{2}: t^{1}\right)=2,\left(t^{1}: t^{0}\right)=0$; the elements of $A$ are $\pm t^{1}$ and $\pm t^{0}$ (the cell complex $K$ is a cell decomposition of the projective plane; then $A$ is a line on this plane). The group $\Delta_{A: K}^{1}$ consists of all elements of the form $\pm 2 n t^{1}$ with an arbitrary 
integer $n$, while the elements of $\Delta_{G: K}^{2}=\Delta_{G}^{2}$ are of the form $n t^{2}$; thus $\Delta_{A: K}^{1}$ and $\Delta_{G: K}^{2}$ are both infinite cyclic.

4. The cells of $K$ are: $\pm t_{1}^{2}, \pm t_{2}^{2} ; \pm t^{1} ; \pm t^{0}$ with the incidence coefficients

$$
\left(t_{1}^{2}: t^{1}\right)=\left(t_{2}^{2}: t^{1}\right)=2 ; \quad\left(t^{1}: t^{0}\right)=0
$$

(two projective planes intersecting in a line); let the cells of $A$ be $\pm t_{1}^{2}, \pm t^{1}$, $\pm t^{0}$. The coefficient-groups are $I$ for $\nabla$ and $\bar{I}$ for $\Delta$. Then $\nabla_{K}^{2}$ is the direct sum of an infinite cyclic group (generated by $t_{1}^{2}$ ) and of a group of order 2 (generated by $t_{1}^{2}-t_{2}^{2}$ ). Correspondingly $\Delta_{K}^{2} \approx \bar{I}+I_{2}$ and

$$
\nabla_{K G}^{2}=\nabla_{G}^{2} \approx I, \quad \Delta_{K A}^{2} \approx I_{2}, \quad \nabla_{A K}^{2}=\nabla_{A}^{2} \approx I_{2} .
$$

The geometric meaning of the annihilation $\Delta_{K}^{2} \supseteq \Delta_{K A}^{2} \perp \nabla_{K G}^{2} \subseteq \nabla_{K}^{2}$ is in this case particularly simple.

Now let $K$ be the same, while the cells of $A$ are $\pm t^{1}, \pm t^{0}$ (that is $A$ is the intersection line of the two planes). Then $\nabla_{A: K}^{1}=\nabla_{A}^{1} \approx I, \nabla_{K G}^{2}=\nabla_{K}^{2} \approx I+I_{2}$, $\nabla_{G}^{2} \approx I+I$, while $\nabla_{G: K}^{2}$ (isomorphic with $\nabla_{A: K}^{1}$ ) is the infinite cyclic group generated by $2\left(t_{1}^{2}-t_{2}^{2}\right)$.

5. Let $\mathbf{K}$ be the three-dimensional (continuous) polyhedron composed by the interior and the surface of an ordinary anchor ring. Let $\mathbf{A}$ be a ring-shaped body (homeomorphic with $\mathbf{K}$ ) lying in the interior of $\mathbf{K}$ and going twice around $\boldsymbol{K}$. We suppose moreover that $\mathbf{A}$ is decomposed in to the simplices of a certain closed subcomplex $A$ of a simplicial decomposition $K$ of $\mathbf{K}$; then $\boldsymbol{\Gamma}=\mathbf{K} \backslash \mathbf{A}$ is decomposed into the simplices of the open subcomplex $G=K \backslash A$ of $K$. The coefficient-groups are $I$ for $\Delta$ and $\bar{I}$ for $\nabla$. Then $\Delta_{K A}^{1}=2 \Delta_{K}^{1}, \Delta_{G K}^{1} \approx \nabla_{K G}^{1} \approx I_{2}$ (the equator of $K$ generates the group $\Delta_{G K}^{1}$; taken twice it bounds on $G$ ). The group $\Delta_{K A}^{1}$ is not a direct summand of the group $\Delta_{K}^{1}$.

6. Let $K$ be a complex which arises when we identify the two poles of an ordinary two-dimensional sphere. $A$ is composed of two parallel circles of this sphere. It follows from (16.4):

$$
\stackrel{2}{\pi_{G}}=\stackrel{1}{\pi_{A: K}}+\stackrel{2}{\pi_{K}}-\stackrel{2}{\pi_{K A}}=2+1-0=3 .
$$

7. In addition to the well known elementary examples illustrating the theorem of Phragmén-Brouwer we illustrate the formula (18.1) by a torus surface $K$ on which two meridians $A_{1}$ and $A_{2}$ form the complex $A$; the whole figure is considered in a cell decomposition in which the two meridians $A_{1}$ and $A_{2}$, oriented in opposite senses, are cells $t_{1}^{1}$ and $t_{2}^{1}$. Then $t_{1}^{1}-t_{2}^{1}$ is a $\nabla$-cycle on $A$ extensible over $K$; therefore $t_{1}^{1}$ and $t_{2}^{1}$ belong to the same class $z^{1} \in \nabla_{A: K}^{1}$; the group $\left(E_{A: K}^{1} \nabla_{Q_{1}}^{1}\right) \cap\left(E_{A: K}^{2} \nabla_{Q_{2}}^{1}\right)$ is generated by this $z^{1}$ and is infinite cyclic. The group $\left(\nabla_{G: G_{1}}^{2} \cap \nabla_{G: G_{2}}^{2}\right) \approx I$ is generated by $t_{1}^{2}-t_{2}^{2}$, where the cells $t_{1}^{2}$ and $t_{2}^{2}$ correspond to the two domains into which the ring surface is decomposed by the meridians $A_{1}$ and $A_{2}$. An open plane circular ring $K$ and two radial segments $A_{1}$ and $A_{2}$ on it could be treated in the same way. 


\section{Chapter II. Spectra}

\section{§5. CELL SPECTRA}

20. Projections. Let $K_{\alpha}$ and $K_{\beta}$ be two cell complexes. Let there be given for each dimension $r$ a homomorphism $\varpi_{\alpha}^{\beta}$ of the group $L_{\beta}^{r}(I)$ of all $r$-dimensional integer chains of $K_{\beta}$ into the group $L_{\alpha}^{r}(I)$ of all $r$-dimensional integer chains of $K_{\alpha}$. We suppose moreover that these homomorphisms commute with the operator $\Delta$, that is, that for any (integer) chain $x_{\beta}^{r}$ on $K_{\beta}$

$$
\Delta \varpi_{\alpha}^{\beta}{\stackrel{r}{x_{\beta}}}^{2}=\varpi_{\alpha}^{\beta} \Delta x_{\beta}^{r} \text {. }
$$

Under these conditions the system of the given homomorphisms $\varpi_{\alpha}^{\beta}$ is called a projection of the cell complex $K_{\beta}$ in to the cell complex $K_{\alpha}$.

Let $A_{\alpha} \subseteq K_{\alpha}, A_{\beta} \subseteq K_{\beta}$ be closed subcomplexes of $K_{\alpha}, K_{\beta}$ respectively. We put $G_{\alpha}=K_{\alpha} \backslash A_{\alpha}, G_{\beta}=K_{\beta} \backslash A_{\beta}$ and write systematically $L_{\alpha}^{r}, L_{\alpha 0}^{r}, L_{\alpha 1}^{r}$ for $L_{K_{\alpha}}^{r}, L_{A_{\alpha}}^{r}, L_{G_{\alpha}}^{r}, \cdots$, and $J_{\alpha 0}^{\alpha}, J_{\alpha 1}^{\alpha}, \cdots$ for $J_{A_{\alpha}}^{K_{\alpha}}, J_{G_{\alpha}}^{K_{\alpha}}, \cdots$.

Any chain on $A_{\alpha}, A_{\beta}$ being a chain on $K_{\alpha}, K_{\beta}$, we say that the projection $\varpi_{\alpha}^{\beta}$ is cogredient (with $A_{\beta}, A_{\alpha}$ ) if every (integer) chain of $A_{\beta}$ is mapped by $\varpi_{\alpha}^{\beta}$ onto a chain on $A_{\alpha}$. As for $x_{\beta}^{\tau}$ on $A_{\beta}, x_{\alpha}^{\tau}$ on $A_{\alpha}$, the boundaries $\Delta x_{\beta}^{\tau}, \Delta x_{\alpha}^{r}$ are the same on $A_{\beta}, A_{\alpha}$ and on $K_{\beta}, K_{\alpha}$, the projection $\omega_{\alpha}^{\beta}$ of $K_{\beta}$ into $K_{\alpha}$ (cogredient with $A_{\beta}, A_{\alpha}$ ) generates a projection $\tilde{\omega}_{\alpha 0}^{\beta 0}$ of $A_{\beta}$ into $A_{\alpha}$.

Now define for any chain $x_{\beta 1}^{r}$ on $G_{\beta}$ :

$$
\tilde{\omega}_{\alpha 1}^{\beta 1} x_{\beta 1}^{r}=J_{\alpha 1}^{\alpha} \varpi_{\alpha}^{\beta} x_{\beta 1}^{r} \text {. }
$$

Then whatever be the chain $x_{\beta}$ on $K_{\beta}, J_{\alpha 1}^{\alpha} \varpi_{\alpha}^{\beta} x_{\beta}=J_{\alpha 1}^{\alpha} \varpi_{\alpha}^{\beta} J_{\beta 1}^{\beta} x_{\beta}$. We use this remark in

$$
\begin{aligned}
\Delta_{\alpha 1} \omega_{\alpha 1}^{\beta 1} x_{\beta 1}^{r} & =\Delta_{\alpha 1} J_{\alpha 1}^{\alpha} \omega_{\alpha}^{\beta} x_{\beta 1}^{r}=J_{\alpha 1}^{\alpha} \Delta_{\alpha} \varpi_{\alpha}^{\beta} x_{\beta 1}^{r}=J_{\alpha 1}^{\alpha} \varpi_{\alpha}^{\beta} \Delta_{\beta} x_{\beta 1}^{r}=J_{\alpha 1}^{\alpha} \varpi_{\alpha}^{\beta} J_{\beta 1}^{\beta} \Delta_{\beta} x_{\beta 1}^{r} \\
& =\varpi_{\alpha 1}^{\beta 1} J_{\beta 1}^{\beta} \Delta_{\beta} x_{\beta 1}^{r}=\varpi_{\alpha 1}^{\beta 1} \Delta_{\beta 1} x_{\beta 1}^{r},
\end{aligned}
$$

where $\Delta_{\alpha}, \Delta_{\beta}, \Delta_{\alpha 1}, \Delta_{\beta 1}$ operate on $K_{\alpha}, K_{\beta}, G_{\alpha}, G_{\beta}$.

20.2. Thus a projection $\varpi_{\alpha}^{\beta}$ of $K_{\beta}$ into $K_{\alpha}$ cogredient with $A_{\beta}, A_{\alpha}$ generates a projection $\varpi_{\alpha 0}^{\beta 0}$ of $A_{\beta}$ into $A_{\alpha}$ (identical on $A_{\beta}$ with $\varpi_{\alpha}^{\beta}$ ) and (by (20.11)) a projection $\varpi_{\alpha 1}^{\beta 1}$ of $G_{\beta}$ in to $G_{\alpha}$.

21. Conjugate homomorphisms. The projection $\varpi_{\alpha}^{\beta}$ of $K_{\beta}$ into $K_{\alpha}$ assigns to each chain $\bar{x}_{\beta}^{r}=\sum a_{j} t_{\beta j}^{r}$ on $K_{\beta}$ (with respect to a given coefficient-group $\overline{\mathfrak{A}}$ ) the chain $\varpi_{\alpha}^{\beta} \bar{x}_{\beta}^{\tau}=\sum_{j} a_{j} \varpi_{\alpha}^{\beta} t_{\beta j}^{\tau}$ on $K$ (with respect to the same coefficient-group $\overline{\mathfrak{Y}})$ and thus produces for any $r$ a homomorphism $\varpi_{\alpha}^{\beta}$ of the group $\bar{L}_{\beta}^{r}=L_{\beta}^{q}(\overline{\mathfrak{Y}})$ in to $\bar{L}_{\alpha}^{r}=L^{r}(\overline{\mathfrak{A}})$ which will still be called a projection. The conjugate homomorphism $\pi_{\beta}^{\alpha}$ of $L_{\alpha}^{r}=L(\mathfrak{U})$ into $L_{\beta}^{r}=L_{\beta}^{r}(\mathfrak{A})$, where $\mathfrak{A} \mid \overline{\mathfrak{A}}$, assigns to any chain $x_{\alpha}^{r} \in L_{\alpha}^{r}$ the chain $\pi_{\beta}^{\alpha} x_{\alpha}^{r} \in L_{\beta}^{r}$ defined by

$$
\left(\pi_{\beta}^{\alpha} x_{\alpha}^{r} \cdot t_{\beta}^{r}\right)=\left(\stackrel{r}{x_{\alpha} \cdot \omega_{\alpha}^{\beta} t_{\beta}^{r}}\right),
$$

whatever $t_{\beta}^{\tau} \in K_{\beta}$. The homomorphisms $\pi_{\beta}^{\alpha}$ commute with $\nabla$ as is seen by 


$$
\begin{aligned}
\left(\nabla \pi_{\beta}^{\alpha} x_{\alpha}^{r} \cdot t_{\beta}^{r+1}\right. & =\left(\pi_{\beta}^{\alpha} x_{\alpha}^{r} \cdot \Delta t_{\beta}^{r+1}\right)=\left(x_{\alpha}^{r} \cdot \varpi_{\alpha}^{\beta} \Delta t_{\beta}^{r+1}\right)=\left(x_{\alpha}^{r} \cdot \Delta \varpi_{\alpha}^{\beta} t_{\beta}^{r+1}\right) \\
& =\left(\nabla x_{\alpha}^{r} \cdot \varpi_{\alpha}^{\beta} t_{\beta}^{r+1}\right)=\left(\pi_{\beta}^{\alpha} \nabla x_{\alpha}^{r} \cdot t_{\beta}^{r+1}\right) .
\end{aligned}
$$

Moreover, if $\varpi_{\alpha}^{\beta}$ is cogredient with $A_{\beta}, A_{\alpha}$ then for a chain $x_{\alpha 1}^{\gamma}$ on $G_{\alpha}$ and any $t_{\beta}^{\tau} \in A_{\beta}$,

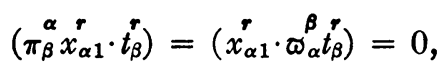

so that $\pi_{\beta}^{\alpha}$ maps $L_{\alpha 1}^{r}$ in to $L_{\beta 1}^{r}$ and thus produces the homomorphism $\pi_{\beta 1}^{\alpha 1}$ of $L_{\alpha 1}^{r}$ into $L_{\beta 1}^{r}$ identical with $\pi_{\beta}^{\alpha}$ on $L_{\alpha 1}^{r} \subseteq L_{\alpha}^{r}$ and conjugate with $\varpi_{\alpha 1}^{\beta 1}$.

On the other hand we have the homomorphism $\pi_{\beta 0}^{\alpha 0}$ conjugate with $\varpi_{\alpha 0}^{\beta 0}$ in the form

$$
\pi_{\beta 0}^{\alpha 0} \underset{x_{\alpha 0}^{r}}{r}=J_{\beta 0}^{\beta} \pi_{\beta}^{\alpha} x_{\alpha 0}^{r} \quad \text { for any } x_{\alpha 0}^{r} \in L_{\alpha 0}^{r} .
$$

We collect all we have just said about the cogredient projections $\tilde{\omega}_{\alpha}^{\beta}$ in the following formulas:

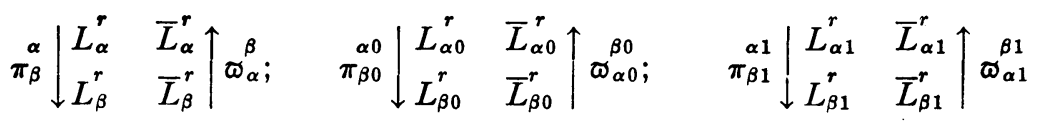

where

$$
\begin{cases}\varpi_{\alpha 0}^{\beta 0}=\varpi_{\alpha}^{\beta} ; & \pi_{\beta 0}^{\alpha 0}=J_{\beta 0}^{\beta} \pi_{\beta}^{\alpha}, \\ \varpi_{\alpha 1}^{\beta 1}=J_{\alpha 1}^{\alpha} \varpi_{\alpha}^{\beta} ; & \pi_{\beta 1}^{\alpha 1}=\pi_{\beta}^{\alpha},\end{cases}
$$

and $\varpi_{\alpha}^{\beta}, \varpi_{\alpha 0}^{\beta 0}, \varpi_{\alpha 1}^{\beta 1}$ commute with $\Delta$, while $\pi_{\beta}^{\alpha}, \pi_{\beta 0}^{\alpha 0}, \pi_{\beta 1}^{\alpha 1}$ commute with $\nabla$. Therefore the homomorphisms $\varpi_{\alpha}^{\beta}, \varpi_{\alpha 0}^{\beta 0}, \varpi_{\alpha 1}^{\beta 1}$ map $\Delta_{\beta}^{r}, \Delta_{\beta 0}^{r}, \Delta_{\beta 1}^{r}$ respectively into $\Delta_{\alpha}^{r}, \Delta_{\alpha 0}^{r}, \Delta_{\alpha 1}^{r}$, while $\pi_{\beta}^{\alpha}, \pi_{\beta 0}^{\alpha 0}, \pi_{\beta 1}^{\alpha 1}$ map $\nabla_{\alpha}^{r}, \nabla_{\alpha 0}^{r}, \nabla_{\alpha 1}^{r}$ in to $\nabla_{\beta}^{r}, \nabla_{\beta 0}^{r}, \nabla_{\beta 1}^{r}$ :

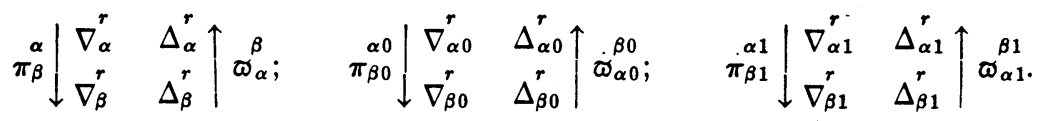

From the definition of $\varpi_{\alpha 0}^{\beta 0}, \varpi_{\alpha 1}^{\beta 1}, \cdots$ follows furthermore:

$$
\begin{array}{rlrl}
\varpi_{\alpha}^{\beta} E_{\beta}^{\beta 0} \bar{x}_{\beta 0}^{r} & =E_{\alpha}^{\alpha 0} \varpi_{\alpha 0}^{\beta 0} \bar{x}_{\beta 0}^{r} ; & \pi_{\beta}^{\alpha} E_{\alpha}^{\alpha 1} x_{\alpha 1}^{r}=E_{\beta}^{\beta 1} \pi_{\beta 1}^{\alpha 1} x_{\alpha 1}^{r}, \\
\pi_{\beta 0}^{\alpha 0} J_{\alpha 0}^{\alpha} x_{\alpha}^{r}=J_{\beta 0}^{\beta} \pi_{\beta}^{\alpha} x_{\alpha}^{r} ; & \varpi_{\alpha 1}^{\beta 1} J_{\beta 1}^{\beta} \bar{x}_{\beta}^{r}=J_{\alpha 1}^{\alpha} \varpi_{\alpha}^{\beta} \bar{x}_{\beta}^{r} .
\end{array}
$$

22. Cell spectra. Two projections $\varpi_{\alpha}^{\beta}$ and $\varpi_{\alpha}^{\prime \beta}$ of $K_{\beta}$ into $K_{\alpha}$ are called homologous to each other if they produce the same homomorphism of $\Delta_{\beta}^{r}$ into $\Delta_{\alpha}^{r}$.

Now let there be given an unbounded partially ordered set of cell complexes $K_{\alpha}$; we write $\beta>\alpha$ if in this set $K_{\beta}$ follows $K_{\alpha}$; "unbounded" means that to each two elements of the set a third element of the same set can be found which follows on each of the two given elements.

Suppose that for any $\beta>\alpha$ a finite number of "allowed" projections $\varpi_{\alpha}^{\beta}$ of $K_{\beta}$ in to $K_{\alpha}$ is given in such a manner that: 
1. Any two allowed projections of $K_{\beta}$ into $K_{\alpha}$ are homologous.

2. If $\gamma>\beta>\alpha$ and $\varpi_{\beta}^{\gamma}, \varpi_{\alpha}^{\beta}$ are allowed projections then the projection $\varpi_{\alpha}^{\gamma}=\varpi_{\alpha}^{\beta}\left(\varpi_{\beta}^{\gamma}\right)$ of $K_{\gamma}$ into $K_{\alpha}$ is allowed.

Under these hypotheses the partially ordered set of the cell complexes $K_{\alpha}$ with their allowed projections $\omega_{\alpha}^{\beta}$ is called a cell spectrum and denoted by

$$
K=\left\{K_{\alpha}, \widetilde{\varpi}_{\alpha}^{\beta}\right\} \text {. }
$$

A cell spectrum (22) determines two group spectra [1, pp. 58-62] or homomorphism systems [13, pp. 668-669]: the inverse group spectrum $\left(\Delta_{\alpha}^{r}, \omega_{\alpha}^{\beta}\right)$ and the direct group spectrum $\left(\nabla_{\alpha}^{r}, \pi_{\beta}^{\alpha}\right)$, the homomorphisms of the two spectra being conjugate. Therefore the limit groups (see section 23) called the Betti groups of the cell spectrum $K$,

$$
\Delta_{K}^{r}=\lim \inf \left(\Delta_{\alpha}^{r}, \underset{\varpi_{\alpha}^{\beta}}{\boldsymbol{\beta}}\right) ; \quad \nabla_{K}^{r}=\lim \sup \left(\nabla_{\alpha}^{r}, \boldsymbol{\pi}_{\beta}^{\alpha}\right),
$$

are dual.

Now let the closed subcomplexes $A_{\alpha} \subseteq K_{\alpha}$ be given in such a way that

1. each allowed projection $\varpi_{\alpha}^{\beta}$ is cogredient with $A_{\beta}, A_{\alpha}$;

2. each two projections $\varpi_{\alpha 0}^{\beta 0}, \varpi_{\alpha 0}^{\prime \beta 0}$ of $A_{\beta}$ into $A_{\alpha} ; \varpi_{\alpha 1}^{\beta 1}, \varpi_{\alpha 1}^{\prime \beta 1}$ of $G_{\beta}$ into $G_{\alpha}$, generated by allowed projections $\varpi_{\alpha}^{\beta}, \varpi_{\alpha}^{\prime \beta}$ of $K_{\beta}$ into $K_{\alpha}$, are homologous to each other. Then the given cell spectrum (22) is called cogredient with the subcomplexes $A_{\alpha}$ and $G_{\alpha}$; such a spectrum defines the cell spectra

$$
A=\left\{A_{\alpha}, \varpi_{\alpha 0}^{\beta 0}\right\} ; \quad G=\left\{G_{\alpha}, \varpi_{\alpha 1}^{\beta 1}\right\}
$$

with their Betti groups

$$
\begin{aligned}
& \Delta_{A}^{r}=\lim \inf \left(\Delta_{\alpha 0}^{r}, \varpi_{\alpha 0}^{\beta 0}\right), \quad \nabla_{A}^{r}=\lim \sup \left(\nabla_{\alpha 0}^{r}, \pi_{\beta 1}^{\alpha 0}\right) ; \\
& \Delta_{G}^{r}=\lim \inf \left(\Delta_{\alpha 1}^{r}, \varpi_{\alpha 1}^{\beta 1}\right), \quad \nabla_{G}^{r}=\lim \sup \left(\nabla_{\alpha 1}^{r}, \pi_{\beta 1}^{\alpha 1}\right)
\end{aligned}
$$

and the dualities

$$
\Delta_{A}^{r}\left|\nabla_{A}^{r} ; \quad \Delta_{G}^{r}\right| \nabla_{G}^{r}
$$

\section{§6. THE EXTENSION AND THE INTERSECTION HOMOMORPHISMS AND} THE GROUPS OF THE FIGURE $K, A, G$ FOR CELL SPECTRA

23. Lemmas on group spectra. In this section "spectrum" means "group spectrum." Let $\left(U_{\alpha}, \pi_{\beta}^{\alpha}\right)$ be a direct spectrum with the limit group $U$. The set theoretical sum of all groups $U_{\alpha}$ is decomposed into bundles or classes of equivalent elements, where two elements $u_{\alpha} \in U_{\alpha}$ and $u_{\beta} \in U_{\beta}$ are called equivalent if there exists a $\gamma>\alpha, \beta$ such that $\pi_{\gamma}^{\alpha} u_{\alpha}=\pi_{\gamma}^{\beta} u_{\beta}$. These bundles are the elements of the limit group $U$. To get the sum of $u=\left\{u_{\alpha}\right\} \in U$ and $u^{\prime}=\left\{u_{\alpha}^{\prime}\right\} \in U$ take $u_{\alpha} \in u, u_{\beta}^{\prime} \in u^{\prime}$ and any $\gamma>\alpha, \beta$. Then $u+u^{\prime}$ is defined as the bundle containing $\pi_{\gamma}^{\alpha} u_{\alpha}+\pi_{\gamma}^{\beta} u_{\beta}$ (see $\left[1\right.$, p. 59]). Let $\left(\bar{U}_{\alpha}, \varpi_{\alpha}^{\beta}\right)$ be an in- 
verse spectrum. The elements of the limit group $\bar{U}=\lim \inf \left(\bar{U}_{\alpha}, \varpi_{\alpha}^{\beta}\right)$ are "threads," that is systems $\bar{u}=\left\{\bar{u}_{\alpha}\right\}$ of elements $\bar{u}_{\alpha} \in \bar{U}_{\alpha}$ such that:

1. the thread $\bar{u}$ contains one and only one element $\bar{u}_{\alpha}$ of each group $\bar{U}_{\alpha}$;

2. if $\bar{u}_{\alpha} \in \bar{u}, \bar{u}_{\beta} \in \bar{u}$ and $\beta>\alpha$ then $\bar{u}_{\alpha}=\varpi_{\alpha}^{\beta} \bar{u}_{\beta}$.

The sum of two threads $\bar{u}=\left\{\bar{u}_{\alpha}\right\}$ and $\bar{u}^{\prime}=\left\{\bar{u}_{\alpha}^{\prime}\right\}$ is the thread $\left\{\bar{u}_{\alpha}+\bar{u}_{\alpha}^{\prime}\right\}$ (see $[1$, p. 58]).

LEMMA 23.1 (obvious). If in an inverse spectrum $\left(\bar{U}_{\alpha}, \varpi_{\alpha}^{\beta}\right)$ the subgroups $\bar{U}_{\alpha 0} \subseteq \bar{U}_{\alpha}$ are given in such a way that $\omega_{\alpha}^{\beta} \bar{U}_{\beta 0} \subseteq \bar{U}_{\alpha 0}$ for $\beta>\alpha$ then the groups $\bar{U}_{\alpha 0}$ with their homomorphisms $\omega_{\alpha 0}^{\beta 0}=\varpi_{\alpha}^{\beta}$ form an inverse spectrum and $\bar{U}_{0}=\lim$ inf $\left(\bar{U}_{\alpha 0}, \widetilde{\omega}_{\alpha 0}^{\beta 0}\right)$ is a subgroup of $\bar{U}=\lim \inf \left(\bar{U}_{\alpha}, \varpi_{\alpha}^{\beta}\right)$.

If in a direct spectrum $\left(U_{\alpha}, \pi_{\beta}^{\alpha}\right)$ the subgroups $U_{\alpha 0} \subseteq U_{\alpha}$ are given in such a way that $\pi_{\beta}^{\alpha} U_{\alpha 0} \subseteq U_{\beta 0}$, then the $U_{\alpha 0}$ with their homomorphisms $\pi_{\beta 0}^{\alpha 0}=\pi_{\beta}^{\alpha}$ form a direct spectrum $\left(U_{\alpha 0}, \pi_{\beta 0}^{\alpha 0}\right)$. Any bundle of this spectrum is contained in a bundle of $\left(U_{\alpha}, \pi_{\beta}^{\alpha}\right)$ and any bundle of $\left(U_{\alpha}, \pi_{\beta}^{\alpha}\right)$ contains at most one bundle of $\left(U_{\alpha 0}, \pi_{\beta 0}^{\alpha 0}\right)$. Therefore the group $U_{0}=\lim \sup \left(U_{\alpha 0}, \pi_{\beta 0}^{\alpha 0}\right)$ is mapped isomorphically on a subgroup of $U=\lim \sup \left(U_{\alpha}, \pi_{\beta}^{\alpha}\right)$ and can be identified with this $\operatorname{subgroup}\left({ }^{3}\right)$.

LEMma 23.2. Under the previous hypotheses the group

$$
\bar{U}_{\beta: \beta 0}=\bar{U}_{\beta}-\bar{U}_{\beta 0} ; \quad U_{\alpha: \alpha 0}=U_{\alpha}-U_{\alpha 0}
$$

is mapped by

$$
\stackrel{\omega}{\alpha}_{\alpha}^{\beta} \text { into } \bar{U}_{\alpha: \alpha 0}=\bar{U}_{\alpha}-\bar{U}_{\alpha 0} ; \quad \pi_{\beta}^{\alpha} \text { into } U_{\beta: \beta 0}=U_{\beta}-U_{\beta 0}
$$

$\operatorname{and}\left({ }^{3}\right)$

$$
\bar{U}-\bar{U}_{0}=\lim \inf \left(\bar{U}_{\alpha: \alpha 0}, \varpi_{\alpha}^{\beta}\right) ; \quad U-U_{0}=\lim \sup \left(U_{\alpha: \alpha 0}, \pi_{\beta}^{\alpha}\right) .
$$

Let us sketch the proof of Lemma 23.2. To prove the left-hand assertion we take any element of $\bar{U}-\bar{U}_{0}$, that is, any class of threads $\bar{u}=\left\{\bar{u}_{\alpha}\right\} \in \bar{U}$ congruent with respect to the subgroup $\bar{U}_{0} \subseteq \bar{U}$, and assign to each thread $\bar{u}=\left\{u_{\alpha}\right\}$ belonging to this class the thread $\left\{\mathfrak{u}_{\alpha}\right\}$ $\in \lim \inf \left(\bar{U}_{\alpha}-\bar{U}_{\alpha 0}, \omega_{\alpha}^{\beta}\right)$ where $\bar{u}_{\alpha} \in \overline{\mathfrak{u}}_{\alpha} \in \bar{U}_{\alpha}-\bar{U}_{\alpha 0}$. This correspondence is easily seen to be an isomorphism between $\bar{U}-\bar{U}_{0}$ and $\lim \inf \left(\bar{U}_{\alpha}-\bar{U}_{\alpha 0}, \omega_{\alpha}^{\beta}\right)$.

To prove the right-hand assertion define the group $U^{\prime}$ as follows. Call the elements $u_{\alpha}$ and $u_{\beta}$ of the set theoretical sum of the groups $U_{\alpha}$ equivalent with respect to $U_{0}$ if a $\gamma>\alpha, \beta$ can be found such that $\pi_{\gamma}^{\alpha} u_{\alpha}-\pi_{\gamma}^{\beta} u_{\beta} \in U_{\gamma 0}$. The set theoretical sum of the groups $U_{\alpha}$ falls now in to classes of elements equivalent with respect to $U_{0}$ and these classes form the group $U^{\prime}$ (we define the sum of two classes $u$ and $u^{\prime}$ as follows: take $u_{\alpha} \in u, u_{\beta}^{\prime} \in u^{\prime}$ and $\gamma>\alpha, \beta$; define $u+u^{\prime}$

(3) In cases when among all isomorphisms between two isomorphic groups a well defined isomorphism is chosen in some "natural" way we often replace the sign of isomorphism by the equality sign and consider the given groups as being identical. 
as the equivalence class containing $\left.\pi_{\gamma}^{\alpha} u_{\alpha}+\pi_{\gamma}^{\beta} u_{\beta}^{\prime}\right)$. The group $U^{\prime}$ is easily seen to be isomorphic with both $U-U_{0}$ and $\lim \sup \left(U_{\alpha}-U_{\alpha 0}, \pi_{\beta}^{\alpha}\right)$.

Lemma 23.3. Let two direct spectra $\left(U_{\alpha}, \rho_{\beta}^{\alpha}\right)$ and $\left(V_{\alpha}, \sigma_{\beta}^{\alpha}\right)$ and two inverse spectra $\left(\bar{U}_{\alpha}, \bar{\rho}_{\alpha}^{\beta}\right)$ and $\left(\bar{V}_{\alpha}, \bar{\sigma}_{\alpha}^{\beta}\right)$ be given [the elements $U_{\alpha}$ and $V_{\alpha}\left(\bar{U}_{\alpha}\right.$ and $\left.\bar{V}_{\alpha}\right)$ of these spectra correspond to each other in (1-1) manner]. For every $\alpha$ let a homomorphism

$$
f_{\alpha} \text { of } U_{\alpha} \text { into } V_{\alpha} ; \quad \bar{f}_{\alpha} \text { of } \bar{V}_{\alpha} \text { into } \bar{U}_{\alpha}
$$

be given in such a way that for every $\beta>\alpha$ and $u_{\alpha} \in U_{\alpha}\left(\bar{v}_{\alpha} \in \bar{V}_{\alpha}\right)$ we have

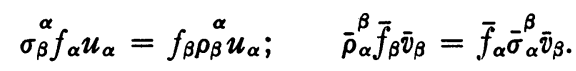

Then we obtain a homomorphism

$$
\begin{aligned}
& \text { f of } U=\lim \sup \left(U_{\alpha}, \rho_{\alpha}^{\beta}\right) \text { into } V=\lim \sup \left(V_{\alpha}, \sigma_{\beta}^{\alpha}\right) \text {; } \\
& \bar{f} \text { of } \bar{V}=\lim \inf \left(\bar{V}_{\alpha}, \bar{\sigma}_{\alpha}^{\beta}\right) \text { into } \bar{U}=\lim \inf \left(\bar{U}_{\alpha}, \bar{\rho}_{\alpha}^{\beta}\right) \\
& \text { by putting in correspondence to each } \\
& \text { bundle } u=\left\{u_{\alpha}\right\} \\
& \text { the bundle } v=f u \\
& \text { containing for } a u_{\alpha} \in u \\
& \text { the element } f_{\alpha} u_{\alpha} \text {. } \\
& \text { thread } \bar{v}=\left\{\bar{v}_{\alpha}\right\} \\
& \text { the thread } u=\bar{f} \bar{v}=\left\{\bar{f}_{\alpha} \bar{v}_{\alpha}\right\} \text {. }
\end{aligned}
$$

The proof of Lemma 23.3 may be left to the reader as well as the proof of the following

Lemma 23.4. Under the hypotheses of Lemma 23.3

$$
\sigma_{\beta}^{\alpha}\left(f_{\alpha} U_{\alpha}\right) \subseteq f_{\beta} U_{\beta} ; \quad \bar{\rho}_{\alpha}^{\beta}\left(\bar{f}_{\beta} \bar{V}_{\beta}\right) \subseteq \bar{f}_{\alpha} \bar{V}_{\alpha}
$$

and (taking into account Lemma 23.1)

$$
\lim \sup \left(f_{\alpha} U_{\alpha}, \sigma_{\beta}^{\alpha}\right)=f U ; \quad \lim \inf \left(\bar{f}_{\alpha} \bar{V}_{\alpha}, \bar{\rho}_{\alpha}^{\beta}\right)=\bar{f} \bar{V} .
$$

The same is true for the kernels of the homomorphisms $f_{\alpha}, f, \bar{f}_{\alpha}, \bar{f}^{\text {: }}$

$$
\rho_{\beta}^{\alpha}\left(f^{-1} 0_{\alpha}\right) \subseteq f_{\beta}^{-1} 0_{\beta} ; \quad \bar{\sigma}_{\alpha}^{\beta}\left(\bar{f}_{\beta}^{-1} \overline{0}_{\beta}\right) \subseteq{\overline{f_{\alpha}}}^{-1} \overline{0}_{\alpha}
$$

(we write $0_{\alpha}, 0_{\beta}, \overline{0}_{\alpha}, \overline{0}_{\beta}$ for the zero elements of $V_{\alpha}, V_{\beta}, \bar{U}_{\alpha}, \bar{U}_{\beta}$ ), and

(23.44) $\quad \lim \sup \left(\bar{f}_{\alpha}^{-1} 0_{\alpha}, \rho_{\beta}^{\alpha}\right)=f^{-1} 0_{V} ; \quad \lim \inf \left(\bar{f}_{\alpha}^{-1} \overline{0}_{\alpha}, \bar{\sigma}_{\alpha}^{\beta}\right)=\bar{f}^{-1} 0_{\bar{U}}$.

Finally we have

Lemma 23.5. If, still under the hypotheses of 23.3, we have for every $\alpha$

$$
f_{\alpha}\left|\begin{array}{ll}
U_{\alpha} & \bar{U}_{\alpha} \uparrow \\
V_{\alpha} & \bar{V}_{\alpha}
\end{array}\right| \bar{f}_{\alpha},
$$


then

$$
f \begin{cases}U & \bar{U} \uparrow \bar{f} . \\ V & \bar{V}\end{cases}
$$

The proof follows from the definition of the scalar product for limit groups $[13$, p. 670 , formula $(6.2)]$, namely:

For any $u=\left\{u_{\alpha}\right\} \in \lim \sup \left(U_{\alpha}, \rho_{\beta}^{\alpha}\right), \bar{u}=\left\{\bar{u}_{\alpha}\right\} \in \lim \inf \left(\bar{U}_{\alpha}, \bar{\rho}_{\alpha}^{\beta}\right)$

$$
(u \cdot \bar{u})=\left(u_{\alpha} \cdot \bar{u}_{\alpha}\right),
$$

where $u_{\alpha}$ is chosen arbitrarily in $u$.

The formulae (23.51) and (23.41) lead to

$$
(f u \cdot \bar{v})=\left(f_{\alpha} u_{\alpha} \cdot \bar{v}_{\alpha}\right)=\left(u_{\alpha} \cdot \bar{f}_{\alpha} \bar{v}_{\alpha}\right)=(u \cdot \bar{f} \bar{v}),
$$

which proves Lemma 23.5.

24. Simplified definitions of the $\nabla$-groups of a cell spectrum. Lemma 23.2 allows the definition of the group $\nabla_{K}^{\gamma}$, where $K$ is a cell spectrum $K=\left\{K_{\alpha}, \varpi_{\alpha}^{\beta}\right\}$, to take the following simple form. Two $\nabla$-cycles $z_{\alpha}^{r}$ and $z_{\beta}^{r}$ of $K_{\alpha}, K_{\beta}$, respectively, belong by definition to the same $\nabla$-class of $K$ if there exists a $\gamma>\alpha, \beta$ such that $\pi_{\gamma}^{\alpha} z_{\alpha}^{r}-\pi_{\gamma}^{\beta} z_{\beta}^{\gamma}$ bounds on $K_{\gamma}$. These $\nabla$-classes are the elements of $\nabla_{K}^{\gamma}$. The sum of two $\nabla$-classes $z^{r}$ and $z^{\prime r}$ is defined as follows: take $z_{\alpha}^{r} \in z^{r}, z_{\beta}^{\prime \prime} \in z^{\prime r}$ and $\gamma>\alpha, \beta$. Then $z^{r}+z^{\prime r}$ is defined as the $\nabla$-class containing $\pi_{\gamma}^{\alpha} z_{\alpha}^{r}+\pi_{\gamma}^{\beta} z_{\beta}^{r}$.

25. The extension and the intersection homomorphisms. The groups of the figure $K, A, G$. By the formulae (21.5) and (21.6) and the Lemmas 23.3 and 23.5 the homomorphisms $E_{\alpha}^{\alpha 0}, E_{\alpha}^{\alpha 1}, J_{\alpha 0}^{\alpha}, J_{\alpha 1}^{\alpha}$ generate the homomorphisms $E_{K}^{A}, E_{K}^{G}, J_{A}^{K}, J_{G}^{K}$ of the corresponding limit groups according to the figure:

$$
J_{A}^{K}\left|\begin{array}{cc}
\nabla_{K}^{r} & \Delta_{K}^{r} \uparrow \\
\nabla_{A}^{r} & \Delta_{A}^{r}
\end{array}\right| E_{K}^{A} ; \quad J_{G}^{K}\left|\begin{array}{cc}
\Delta_{K}^{r} & \nabla_{K}^{r} \\
\Delta_{G}^{r} & \nabla_{G}^{r}
\end{array}\right| E_{K}^{G} .
$$

According to Lemma 23.4 (and Lemma 23.2 used in (25.3)) define:

$$
\begin{aligned}
& \left\{\begin{aligned}
\nabla_{A K}^{r}=J_{A}^{K} \nabla_{K}^{r} & =\lim \sup \left(\nabla_{A_{\alpha} K_{\alpha}}^{r}, \pi_{\beta 0}^{\alpha 0}\right) ; \\
\Delta_{K A}^{r} & =E_{K}^{A} \Delta_{A}^{r}=\lim \inf \left(\Delta_{K_{\alpha} A}^{r}, \varpi_{\alpha}^{\beta}\right), \\
\Delta_{G K}^{r}=J_{G}^{K} \Delta_{K}^{r} & =\lim \inf \left(\Delta_{G_{\alpha} K_{\alpha}}^{r}, \varpi_{\alpha 1}^{\beta 1}\right) ; \\
\nabla_{K G}^{r} & =E_{K}^{G} \nabla_{G}^{r}=\lim \sup \left(\nabla_{K_{\alpha} G_{\alpha}}^{r}, \pi_{\beta}^{\alpha}\right),
\end{aligned}\right. \\
& \left\{\begin{array}{c}
\Delta_{A: K}^{r}=\left(E_{K}^{A}\right)^{-1} 0_{K}=\lim \inf \left(\Delta_{A_{\alpha}: K_{\alpha}}^{r}, \varpi_{\alpha 0}^{\beta 0}\right) ; \\
\nabla_{G: K}^{r}=\left(E_{K}^{G}\right)^{-1} 0_{K}=\lim \sup \left(\nabla_{G_{\alpha}: K_{\alpha}, \pi_{\beta 1}^{\alpha 1}}^{r}\right), \\
\nabla_{A: K}^{r}=\nabla_{A}^{r}-\nabla_{A K}^{r}=\lim \sup \left(\Delta_{\left.A_{\alpha}: K_{\alpha}, \pi_{\beta 0}\right) ;}^{r}\right) \\
\Delta_{G: K}^{r}=\Delta_{G}^{r}-\Delta_{G K}^{r}=\lim \inf \left(\Delta_{G_{\alpha}: K_{\alpha}}^{r}, \varpi_{\alpha 1}^{\beta 1}\right) .
\end{array}\right.
\end{aligned}
$$

Then by Lemma 23.4 and the formula (12.4): 


$$
\begin{aligned}
\left(J_{A}^{K}\right)^{-1} 0_{A}=\lim \sup \left(\nabla_{K_{\alpha} G_{\alpha}}^{r}, \pi_{\beta}^{\alpha}\right)=\nabla_{K G}^{r} ; \\
\left(J_{G}^{K}\right)^{-1} 0_{G}=\lim \inf \left(\Delta_{K_{\alpha} A_{\alpha}}^{r}, \varpi_{\alpha}^{\beta}\right)=\Delta_{K A}^{r} .
\end{aligned}
$$

From these formulae we get literally as in section 14 the relations (14.1)(14.2) for the spectra $K, A, G$.

26. A direct definition of the groups of the figure $K, A, G$. Since any $\Delta$-cycle $\left(\Delta\right.$-homology) on $A_{\alpha}$ is a $\Delta$-cycle (a $\Delta$-homology) on $K_{\alpha}$, to any thread $\bar{z}_{A}^{r}=\left\{Z_{\alpha 0}^{r}\right\} \in \Delta_{A}^{r}$ corresponds the thread $\bar{z}_{K}^{r}=\left\{B_{\alpha}^{r}\right\} \in \Delta_{K}^{r}$ where $B_{\alpha}^{r}$ is completely determined by $\Im_{\alpha 0}^{r} \subseteq \nexists_{\alpha}^{r}$. This thread $\bar{z}_{K}^{r}$ is the thread $E_{K}^{A} \bar{z}_{A}^{r}$.

Since any $\nabla$-cycle $\left(\nabla\right.$-homology) on $G_{\alpha}$ is a $\nabla$-cycle $\left(\nabla\right.$-homology) on $K_{\alpha}$, any $\nabla$-class (section 24) $z_{G}^{r} \in \nabla_{G}^{r}$ is a subset of a well determined $\nabla$-class $z_{K}^{r} \in \nabla_{K}^{r}$, and this $z_{K}^{r}$ is the $\nabla$-class $E_{K}^{G} z_{G}^{r}$.

Let $\bar{z}_{K}^{r}=\left\{\Im_{\alpha}^{r}\right\} \in \Delta_{K}^{r}$. Then $\left\{J_{\alpha 1}^{\alpha} \Re_{\alpha}^{r}\right\}$ is a thread and this thread is the thread $J_{G}^{K} \bar{z}_{K}^{r} \in \Delta_{G}^{r}$.

Let $z_{K}^{r}=\left\{z_{\alpha}^{r}\right\} \in \nabla_{K}^{r}$. Then all $J_{\alpha 0}^{\alpha} z_{\alpha}^{r}$ are elements of the same $\nabla$-class $z_{A}^{r} \in \nabla_{A}^{r}$ and this $\nabla$-class is $J_{A}^{K} z_{K}^{T}$.

Thus we get the following direct definitions of the groups of the figure $K, A, G$ :

The elements of $\Delta_{K A}^{r} \subseteq \Delta_{K}^{r}$ are threads $\bar{z}_{K}^{r}=\left\{B_{\alpha}^{r}\right\} \in \Delta_{K}^{r}$ containing threads $\bar{z}_{A}^{r}=\left\{Z_{\alpha 0}^{r}\right\} \in \Delta_{A}^{r}$ in the sense that each $\Delta$-class $Z_{\alpha 0}^{r}$ is a subset of the corresponding $\Delta$-class $\mathfrak{B}_{\alpha}^{r}$.

The elements of $\nabla_{K G}^{r}$ are $\nabla$-classes $z_{K}^{r} \in \nabla_{K}^{r}$ containing as subsets $\nabla$-classes $z_{G}^{r} \in \nabla_{G}^{r}$.

The elements of $\nabla_{A K}^{r} \subseteq \nabla_{A}^{r}$ are $\nabla$-classes $z_{A}^{r} \in \nabla_{A}^{r}$ containing among their elements extensible $\nabla$-cycles, that is, $\nabla$-cycles $z_{\alpha 0}^{r}$ on $A_{\alpha}$ of the form $z_{\alpha 0}^{r}=J_{\alpha 0}^{\alpha} z_{\alpha}^{r}$ where $z_{\alpha}^{r}$ is a $\nabla$-cycle on $K_{\alpha}$. For any element $z_{\alpha 0}^{r}$ of $z_{A}^{r} \in \nabla_{A K}^{r}$ there is a $\beta>\alpha$ such that $\pi_{\beta 0}^{\alpha 0} z_{\alpha 0}$ is extensible.

The elements of $\Delta_{G K}^{r} \subseteq \Delta_{G}^{r}$ are "extensible threads" $z_{G}^{r}=\left\{z_{\alpha \alpha 1}^{r}\right\} \in \Delta_{G}^{r}$, that is, threads whose elements $z_{\alpha 1}^{r}$ are all extensible (that is of the form $z_{\alpha 1}^{r}=J_{\alpha 1 \delta_{\alpha}^{\alpha}}^{\alpha}$ where $z^{r} \in \Delta_{\alpha}^{r}$.

The elements of $\Delta_{A: K}^{r} \subseteq \Delta_{A}^{r}$ are threads $z_{A}^{r}=\left\{z_{\alpha \alpha 0}^{r}\right\} \in \Delta_{A}^{r}$ bounding on $K$ in the sense that the elements of each $\Delta$-class ${ }_{\alpha \alpha 0}^{r}$ bound on $K_{\alpha}$.

The elements of $\nabla_{G: K}^{r} \subseteq \nabla_{G}^{r}$ are $\nabla$-classes $z_{G}^{r} \in \nabla_{G}^{r}$ bounding on $K$ in the sense that $z_{G}^{\tau}$ is a subset of the $\nabla$-class $z_{K}^{\tau}=0$ representing the zero of the group $\nabla_{K}^{r}$. In other words, for any $z_{\alpha 1}^{\tau} \in z_{G}^{\tau}$ there is a $\pi_{\beta 1}^{\alpha 1} z_{\alpha 1}^{r}$ bounding on $K_{\beta}$.

Definition 26.1. The $\nabla$-cycle $z_{\alpha 0}^{r}$ on $A_{\alpha}$ and the $\nabla$-cycle $z_{\beta 0}^{r}$ on $A_{\beta}$ belong to the same extension class (with respect to the cell spectrum $K$ ) if there exists a $\gamma>\alpha, \beta$ such that $\pi_{\gamma 0}^{\alpha 0} z_{\alpha 0}^{r}-\pi_{\gamma 0}^{\beta 0} z_{\beta 0}^{r}$ is extensible.

Definition 26.2. The threads $\bar{z}_{G}^{r}=\left\{B_{\alpha 1}^{r}\right\}$ and $\bar{z}_{G}^{\prime r}=\left\{B_{\alpha 1}^{\prime r}\right\}$ belong to the same extension class if for each $\alpha$ the $\Delta$-class ${ }_{\gamma_{\alpha 1}^{r}}^{r}-\gamma_{\alpha 1}^{\prime r}$ is extensible (that is, is of the form ${ }_{\gamma_{\alpha 1}}^{\tau}-\xi_{\alpha 1}^{\prime r}=J_{\alpha 1 \delta_{\alpha}^{\alpha}}^{\alpha}$ with $z_{\alpha}^{\tau} \in \Delta_{\alpha}^{\tau}$ ). 
Now the elements of $\nabla_{A: K}^{r}=\nabla_{A}^{r}-\nabla_{A K}^{r}$ can be identified with the extension classes of $\nabla$-cycles $z_{\alpha 0}^{r}$. To get the sum of two extension classes $z^{r}$ and $z^{\prime r}$ take as always $z_{\alpha 0} \in z^{r}, z_{\beta 0}^{\prime r} \in z^{\prime r}$, choose any $\gamma>\alpha, \beta$ and define $z^{r}+z^{\prime r}$ as the extension class containing $\pi_{\gamma 0}^{\alpha 0} z_{\alpha 0}^{r}+\pi_{\gamma 0}^{\beta 0} z_{\beta 0}^{r}$; the group $\nabla_{A: K}^{r}$ can be defined as the group of the extension classes of $r$-dimensional $\nabla$-cycles on $A_{\alpha}$ ( $\alpha$ variable) with this addition.

The elements of the group $\Delta_{G: K}^{r}$ are extension classes of threads $z_{G}^{r} \in \Delta_{G}^{r}$.

27. Proof of the third pair of isomorphisms. Let

$$
z_{A}^{r}=\left\{z_{\alpha 0}^{r}\right\} \in \nabla_{A: K}^{r}
$$

The $\nabla$-cycle $\nabla E_{\alpha}^{\alpha 0} z_{\alpha 0}^{\tau}$ is on $G_{\alpha}$ and bounds on $K_{\alpha}$.

27.1. If $z_{\alpha 0}$ and $z_{\beta 0}$ belong to the same extension class, that is, if for a certain $\gamma>\alpha, \beta$

$$
\underset{\pi_{\gamma 0}^{\alpha 0} z_{\alpha 0}^{r}}{\alpha \pi_{\gamma 0}^{\beta 0} z_{\beta 0}}=J_{\gamma 0}^{\gamma} z_{\gamma}^{r},
$$

where $z_{\gamma}$ is a $\nabla$-cycle on $K_{\gamma}$, then

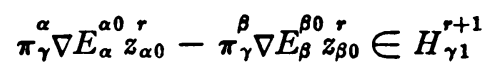

( $H_{\gamma 1}^{r}$ is the group of all $r$-dimensional $\nabla$-cycles on $G_{\gamma}$ bounding on $G_{\gamma}$ ).

To prove (27.12) we first deduce from (27.11) and section 15:

$$
\nabla E_{\gamma \pi_{\gamma 0} z_{\alpha 0}}^{\gamma 0}-\nabla E_{\gamma}^{\gamma 0} \pi_{\gamma 0}^{\beta 0} z_{\beta 0}^{r} \in H_{\gamma 1}^{r+1} .
$$

On the other hand

$$
\pi_{\gamma}^{\alpha} \nabla E_{\alpha}^{\alpha 0} z_{\alpha 0}^{r}-\nabla E_{\gamma}^{\gamma 0} \pi_{\gamma 0}^{\alpha 0} z_{\alpha 0}=\nabla\left(\pi_{\gamma}^{\alpha} E_{\alpha}^{\alpha 0} z_{\alpha 0}^{r}-E_{\gamma}^{\gamma 0} \pi_{\beta 0}^{\alpha 0} z_{\alpha 0}^{r}\right)
$$

and $\pi_{\gamma}^{\alpha} E_{\alpha}^{\alpha 0} z_{\alpha 0}^{\gamma}-E_{\gamma}^{\gamma 0} \pi_{\gamma 0}^{\alpha 0} z_{\alpha 0}^{r}$ is on $G_{\gamma}$. Thus

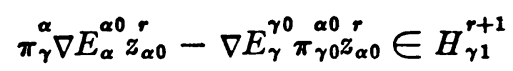

and in the same way

$$
\stackrel{\beta}{\pi_{\gamma} \nabla} \nabla E_{\gamma}^{\beta 0} z_{\beta 0}^{r}-\nabla E_{\gamma}^{\gamma 0} \pi_{\gamma 0}^{\beta 0} z_{\beta 0}^{r} \in H_{\gamma 1}^{r+1} .
$$

From (27.13)-(27.15) follows (27.12) and therefore (27.1).

Thus the operators $\nabla E_{\alpha}^{\alpha 0}$ generate a homomorphism-we call it $\nabla E$ - of $\nabla_{A: K}^{r}$ in to $\nabla_{G: K}^{r+1}$.

27.2. The homomorphism $\nabla E$ maps $\nabla_{A: K}^{r}$ onto $\nabla_{G: K}^{r+1}$. To prove this let $z_{G}^{+1} \in \nabla_{G: K}^{r+1}$ and $z_{\alpha 1}^{+1} \in z_{G}^{+1}$. We are seeking a $\beta>\alpha$ and a $\nabla$-cycle $z_{\beta 0}$ on $A_{\beta}$ such that

$$
\nabla E_{\beta}^{\beta 0} z_{\beta 0}^{r}-\pi_{\beta 1}^{\alpha 1} z_{\alpha 1}^{r+1} \in H_{\beta 1}^{r+1} .
$$

From $z_{\alpha 1}^{+1} \in z_{G}^{+1} \in \nabla_{G: K}^{r+1}$ and the definition of $\nabla_{G: K}^{r+1}$ it follows that there exist a $\beta>\alpha$ and a chain $x_{\beta}^{r}$ on $K_{\beta}$ such that $\nabla x_{\beta}^{\gamma}=\pi_{\beta 1}^{\alpha 1} z_{\alpha 1}^{+1}$. As $\nabla x_{\beta}^{\gamma}$ is on $G_{\beta}$, the chain 
$J_{\beta 0}^{\beta} x_{\beta}^{r}=z_{\beta 0}^{r}$ is a $\nabla$-cycle on $A_{\beta}$, while $x_{\beta}^{\tau}-E_{\beta}^{\beta 0} J_{\beta 0}^{\beta} x_{\beta}^{r}$ is on $G$ and

$$
\nabla\left(x_{\beta}^{r}-E_{\beta}^{\beta 0} J_{\beta 0}^{\beta} x_{\beta}^{r}\right)=\nabla x_{\beta}^{r}-\nabla E_{\beta}^{\beta 0} J_{\beta 0}^{\beta} x_{\beta}^{r}=\pi_{\beta 1}^{\alpha 1} z_{\alpha 1}^{r+1}-\nabla E_{\beta}^{\beta 0} z_{\beta 0}^{r}
$$

from which follows (27.21) and thus (27.2).

27.3. $\nabla E$ maps $\nabla_{A: K}^{r}$ on $\nabla_{G: K}^{r+1}$ isomorphically. Suppose that the element $z_{A}^{r} \in \nabla_{A: K}^{r}$ is mapped by $\nabla E$ on the zero element of the group $\nabla_{G: K}^{r+1}$. We shall prove that the extension class $z_{A}^{r}$ is the zero element of the group $\nabla_{A: K}^{r}$. Take $z_{\alpha 0}^{r} \in z_{A}^{r}$. It follows from our hypotheses that there exists such a $\beta>\alpha$ that

Since

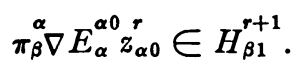

$$
\stackrel{\alpha}{\pi_{\beta} \nabla E_{\alpha}^{\alpha 0} z_{\alpha 0}^{r}}-\nabla E_{\beta}^{\beta 0} \pi_{\beta 0}^{\alpha 0} z_{\alpha 0}^{r} \in H_{\beta 1}^{r+1}
$$

(see (27.14)) and $\pi_{\beta 0}^{\alpha 0} z_{\alpha 0}^{r} \in z_{A}^{r}$, we may suppose from the beginning (replacing $\beta$ by $\alpha$ and $\pi_{\beta 0}^{\alpha 0} z_{\alpha 0}^{r}$ by $z_{\alpha 0}^{r}$ ) that for some $z_{\alpha 0}^{r} \in z_{A}^{\tau}$

$$
\nabla E_{\alpha}^{\alpha 0} z_{\alpha 0}^{r} \in H_{\alpha 1}^{r+1} \text {. }
$$

To deduce from that the identity $z_{A}^{r}=0$ we have only to prove that $z_{\alpha 0}^{r}$ is extensible. To this end take a chain $x_{\alpha 1}^{r}$ on $G_{\alpha}$ bounded by $\nabla E_{\alpha}^{\alpha 0} z_{\alpha 0}^{r}$. Then $z_{\alpha}^{r}=E_{\alpha}^{\alpha 0} z_{\alpha 0}^{r}-x_{\alpha 1}^{r}$ is a $\nabla$-cycle on $K_{\alpha}$ and $J_{\alpha 0}^{\alpha} z_{\alpha}=z_{\alpha 0}^{r}$, q.e.d.

\section{\$7. Simplicial SPECTRA}

28. Nerves. We denote by $N, N_{\alpha}, N_{\beta}, \cdots$ and call nerves the finite complete simplicial complexes (complete means every face of an element of $N$ is itself an element of $N$ ). The oriented simplices of $N$ form a cell complex denoted by $N$ too. and

Let $N_{\alpha}, N_{\beta}$ be nerves, $C_{\alpha}, C_{\beta}$ closed subcomplexes of $N_{\alpha}, N_{\beta}$, respectively,

$$
K_{\alpha}=N_{\alpha} \backslash C_{\alpha}, \quad K_{\beta}=N_{\beta} \backslash C_{\beta} .
$$

Let $\sigma_{\alpha}^{\beta}$ be a simplicial mapping of $N_{\beta}$ into $N_{\alpha}$ such that the image of $C_{\beta}$ under $\sigma_{\alpha}^{\beta}$ is contained in $C_{\alpha}$ :

$$
{ }_{\sigma_{\alpha}}^{\beta} C_{\beta} \subseteq C_{\alpha} .
$$

Then in a well known way $\sigma_{\alpha}^{\beta}$ generates a projection denoted also by $\sigma_{\alpha}^{\beta}$ of the cell complex $N_{\beta}$ into the cell complex $N_{u}$; for any oriented simplex $t_{\beta}^{r}=\left(e_{0}^{\beta} \cdots e_{r}^{\beta}\right)$ of $N_{\beta}$ we put $\sigma_{\alpha}^{\beta} t_{\beta}^{r}=\left(\sigma_{\alpha}^{\beta} e_{0}^{\beta} \cdots \sigma_{\alpha}^{\beta} e_{r}^{\beta}\right)$ if the vertices $\sigma_{\alpha}^{\beta} e_{0}^{\beta}, \cdots, \sigma_{\alpha}^{\beta} e_{r}^{\beta}$ are all different, and $\sigma_{\alpha}^{\beta} t_{\beta}^{r}=0$ if for some $i \neq j, \sigma_{\alpha}^{\beta} e_{i}^{\beta}=\sigma_{\alpha}^{\beta} e_{j}^{\beta}$, whereupon for any chain $x_{\beta}^{r}=\sum a_{i} t_{\beta i}^{\tau}, \sigma_{\alpha}^{\beta} x_{\beta}^{\tau}=\sum a_{i} \sigma_{\alpha}^{\beta} t_{\beta i}^{r}$. The projection $\sigma_{\alpha}^{\beta}$ of $N_{\beta}$ into $N_{\alpha}$ generates a projection $\omega_{\alpha}^{\beta}$ of $K_{\beta}$ into $K_{\alpha}$ by setting

$$
\tilde{\omega}_{\alpha}^{\beta} x^{r}=J_{K_{\alpha} \sigma_{\alpha}{ }^{\beta} x_{\beta}^{r}}^{r} \quad \text { for any } x_{\beta}^{r} \in L_{\beta}^{r}=L_{K_{\beta}}^{r}
$$

(see section 20 where $K_{\alpha}, G_{\alpha}, \omega_{\alpha}^{\beta}, \varpi_{\alpha 1}^{\beta 1}$ are to be replaced by $N_{\alpha}, K_{\alpha}, \sigma_{\alpha}^{\beta}$, $\varpi_{\alpha}^{\beta}$, respectively). 
A known fact is $\left({ }^{4}\right)$ :

LEMMA 28.1. Let $\sigma_{\alpha}^{\beta}$ and $\sigma_{\alpha}^{\prime \beta}$ be two simplicial mappings of $N_{\beta}$ into $N_{\alpha}$ both satisfying the condition (28) and the condition:

(28.11) For every simplex $T_{\beta} \in N_{\beta}$ there exists a simplex $T_{\alpha} \in N_{\alpha}$ containing among its vertices all vertices of both $\sigma_{\alpha}^{\beta} T_{\beta}$ and $\sigma_{\alpha}^{\prime \beta} T_{\beta}$; if in particular $T_{\beta} \in C_{\beta}$ then $T_{\alpha}$ can be chosen among the simplices of $C_{\alpha}$.

Under these hypotheses the projections $\omega_{\alpha}^{\beta}$ and $\varpi_{\alpha}^{\prime \beta}$ of $K_{\beta}$ into $K_{\alpha}$ generated by $\sigma_{\alpha}^{\beta}$ and $\sigma_{\alpha}^{\prime \beta}$ are homologous to each other.

The proof is as follows. Let $e_{1}, e_{2}, \cdots, e_{s}$ be all vertices of $N_{\beta}$ in a definite order. Take a copy $N_{\beta}^{\prime}$ of $N_{\beta}$ with the vertices $e_{1}^{\prime}, e_{2}^{\prime}, \cdots, e_{s}^{\prime}$ and consider all simplices of the form $\left|e_{i_{0}}^{\prime} \cdots e_{i_{p}}^{\prime} e_{i_{p}} \cdots e_{i_{r}}\right|$, where $p=0,1, \cdots, r$ and $\left|e_{i_{0}} \cdots e_{i_{r}}\right|$ is any simplex of $N_{\beta}$. The simplices just defined and their faces form a nerve $N_{\beta}^{\prime \prime}$ called the prism over $N_{\beta}$. Now define for each oriented simplex $t_{\beta}^{r}=\left(e_{i_{0}} \cdots e_{i_{r}}\right)$ of $N_{\beta}$ the prism over $t_{\beta}^{r}$ as the chain

$$
\Pi t_{\beta}^{r}=\sum_{p=0}^{p=r}(-1)^{p}\left(e_{i_{0}}^{\prime} \cdots e_{i_{p}}^{\prime} e_{i_{p}} \cdots e_{i_{r}}\right)
$$

while the prism over the chain $x_{\beta}^{r}=\sum a_{i} t_{\beta i}^{r} \in L_{N_{\beta}}^{r}$ is by definition the chain

$$
\Pi x_{\beta}^{r}=\sum a_{i} \Pi t_{\beta i}^{r}
$$

on $N_{\beta}^{\prime \prime}$. An easy calculation shows: If $z_{\beta}^{r}$ is a $\Delta$-cycle on $K_{\beta}$ then

$$
\Delta \Pi z_{\beta}^{r}=z_{\beta}^{r}-z_{\beta}^{\prime r}-\Pi \Delta z_{\beta}^{r},
$$

where $z_{\beta}^{\prime r}$ is the copy of $z_{\beta}^{r}$ on $N_{\beta}^{\prime}$ and $\Delta$ operates on $N_{\beta}^{\prime \prime}$ at the left and on $N_{\beta}$ at the right (see $[4$, p. 199]).

Now map the vertices of $N_{\beta}$ by $\sigma_{\alpha}^{\beta}$ and those of $N_{\beta}^{\prime}$ by $\sigma_{\alpha}^{\prime \beta}$. By virtue of (28.11) we get in this way a simplicial mapping $\sigma_{\alpha}^{\prime \prime \beta}$ of $N_{\beta}^{\prime \prime}$ in to $N_{\alpha}$. Denoting by $x_{\alpha}^{r+1}$ the chain $\sigma_{\alpha}^{\prime \prime \beta} \Pi z_{\beta}^{r}$ (on $N_{\alpha}$ ), by $x_{\alpha}^{r}$ the chain $\sigma_{\alpha}^{\prime \prime \beta} \Pi \Delta z_{\beta}^{r}$ which is on $C_{\alpha}$. we get

$$
\Delta_{N_{\alpha}} x_{\alpha}^{r+1}=\sigma_{\alpha}^{\beta} z_{\beta}^{r}-\sigma_{\alpha}^{\prime \beta} z_{\beta}^{r}-x_{\alpha}^{r}
$$

and, $K_{\alpha}$ being open on $N_{\alpha}$,

$$
\Delta_{K_{\alpha}} J_{K_{\alpha}}^{N_{\alpha}} x_{\alpha}^{r+1}=J_{K_{\alpha}}^{N_{\alpha}} \Delta_{N_{\alpha}} x_{\alpha}^{r+1}=J_{K_{\alpha} \sigma_{\alpha} z_{\beta}}^{N_{\alpha}{ }^{\beta}}-J_{K_{\alpha}}^{N_{\alpha} \sigma_{\alpha}^{\prime \beta} z_{\beta}^{r}}=\varpi_{\alpha}^{\beta} z_{\beta}^{r}-\varpi_{\alpha}^{\prime \beta} z_{\beta}^{r}
$$

q.e.d.

In the next chapter (section 31 ) we need the following

Corollary 28.2. Let $A_{\alpha}, A_{\beta}$ be closed subcomplexes of $K_{\alpha}=N_{\alpha} \backslash C_{\alpha}$, $K_{\beta}=N_{\beta} \backslash C_{\beta}$; let $\sigma_{\alpha}^{\beta}$, $\sigma_{\alpha}^{\prime \beta}$ map $C_{\beta}$ into $C_{\alpha}$ as well as $A_{\beta}$ into $A_{\alpha}$. Suppose the con-

(4) We write $T^{r}=\left|e_{0} \cdots e_{r}\right|$ for non-oriented and $t^{r}=\left(e_{0} \cdots e_{r}\right)$ for oriented simplices. 
dition (28.11) satisfied; suppose moreover that for $T_{\beta} \in A_{\beta}$ the simplex $T_{\alpha}$ of the latter condition can always be chosen in $A_{\alpha}$. Under these hypotheses not only the projections $\varpi_{\alpha}^{\beta}$ and $\varpi_{\alpha}^{\prime \beta}$ of $K_{\beta}$ into $K_{\alpha}$ generated by $\sigma_{\alpha}^{\beta}$, $\sigma_{\alpha}^{\prime \beta}$ are homologous but the same is true for the corresponding projections $\varpi_{\alpha 0}^{\beta 0}, \varpi_{\alpha 0}^{\prime \beta 0}$ and $\varpi_{\alpha 1}^{\beta 1}, \varpi_{\alpha 1}^{\prime \beta 1}$ of $A_{\beta}$ into $A_{\alpha}$ and of $G_{\beta}$ into $G_{\alpha}$.

To prove the assertion concerning $A_{\beta}, A_{\alpha}$ replace in $28.1 N_{\beta}, N_{\alpha}$ by $A_{\beta} \cup C_{\beta}, A_{\alpha} \cup C_{\alpha}$ and $K_{\beta}, K_{\alpha}$ by $A_{\beta}, A_{\alpha}$. To prove the assertion concerning $G_{\beta}, G_{\alpha}$ replace in $28.1 C_{\beta}, C_{\alpha}$ by $C_{\beta} \cup A_{\beta}, C_{\alpha} \cup A_{\alpha}$ and $K_{\beta}, K_{\alpha}$ by $G_{\beta}, G_{\alpha}$.

29. Simplicial spectra. Let an unbounded partially ordered set of nerves $N_{\alpha}$ be given; we write $\beta>\alpha$ if in this set $N_{\beta}$ follows on $N_{\alpha}$. Let (for each $\alpha$ ) $C_{\alpha}$ be a fixed closed subcomplex of $N_{\alpha}$. We suppose that for each pair $\beta>\alpha$ a finite number of simplicial mappings $\sigma_{\alpha}^{\beta}$ (called projections) of $N_{\beta}$ into $N_{\alpha}$ is given and that the following conditions are satisfied:

1. $\sigma_{\alpha}^{\beta}$ maps $C_{\beta}$ into $C_{\alpha}$;

2. for each pair $\beta>\alpha$ the projections $\sigma_{\alpha}^{\beta}$ satisfy the conditions (28.11);

3. if $\gamma>\beta>\alpha$ and $\sigma_{\beta}^{\gamma}, \sigma_{\alpha}^{\beta}$ are projections, then the simplicial mapping $\sigma_{\alpha}^{\gamma}=\sigma_{\alpha}^{\beta} \sigma_{\beta}^{\gamma}$ of $N_{\gamma}$ into $N_{\alpha}$ is a projection.

Under these hypotheses the partially ordered set of nerves $N_{\alpha}$, of their subcomplexes $C_{\alpha}$ and of the projections $\sigma_{\alpha}^{\beta}$ is called a simplicial spectrum

$$
N=\left\{N_{\alpha}, C_{\alpha}, \sigma_{\alpha}^{\beta}\right\}
$$

A simplicial spectrum (29.1) generates according to section 28 the cell spectrum

$$
K=\left\{K_{\alpha}, \varpi_{\alpha}^{\beta}\right\}
$$

where by $K_{\alpha}$ is denoted the open subcomplex $N_{\alpha} \backslash C_{\alpha}$ as well as the cell complex of all oriented simplices of this open subcomplex; the projections $\boldsymbol{\omega}_{\alpha}^{\beta}$ are (as defined in section 28):

$$
\stackrel{\beta}{\varpi_{\alpha}} x_{\beta}^{r}=J_{K_{\alpha} \sigma_{\alpha} x_{\beta}}^{N_{\alpha}} \quad \text { for any } \dot{x}_{\beta}^{r} \in L_{\beta}^{r}=L_{K_{\beta}}^{r}
$$

REMARK. The complexes $C_{\alpha}$ are called special subcomplexes of the spectrum $N$; if they vanish the spectrum is called bicompact.

\section{Chapter III. Spaces}

\section{§8. THE CELL SPECTRUM AND THE BETTI GROUPS} OF A LOCALLY BICOMPACT SPACE

30. The simplicial and the cell spectrum of a locally bicompact space. The nerve $N$ is called the nerve of the finite system

$$
\alpha=\left\{A_{1}, \cdots, A_{8}\right\}
$$

of subsets $A_{i}$ (of the given set $R$ ) if the vertices $e_{1}, \cdots, e_{s}$ of $N$ are in (1-1)- 
correspondence with the elements $A_{1}, \cdots, A_{s}$ of the system $\alpha$ and any $e_{i_{0}}, \cdots, e_{i_{r}}$ are vertices of a simplex of $N$ if and only if the associate sets $A_{i_{0}}, \cdots, A_{i_{r}}$ have a non-vacuous intersection $\left(^{(5)}\right.$.

Now let $\mathbf{K}$ be a locally bicompact Hausdorff space. We consider the set of all finite coverings,

$$
\alpha=\left\{o_{\alpha 1}, \cdots, o_{\alpha s}\right\},
$$$$
s=s_{\alpha},
$$

of $\mathbf{K}$ by open sets $o_{\alpha 1}, \cdots, o_{\alpha s}$. The nerve of $\alpha$ is denoted by $N_{\alpha}$. We denote by $C_{\alpha}$ the subcomplex of $N_{\alpha}$ consisting of all simplices of $N_{\alpha}$ whose vertices correspond to sets $o_{\alpha i} \in \alpha$ having a non-bicompact closure $\bar{o}_{\alpha i}$ in $\mathbf{K}$. Thus the open subcomplex $K_{\alpha}=N_{\alpha} \backslash C_{\alpha}$ is the subcomplex of all simplices of $N_{\alpha}$ which have among their vertices at least one corresponding to a set $o_{\alpha i} \in \alpha$ with bicompact closure. The set of all $N_{\alpha}$ is partially ordered: we set $\beta>\alpha$ if $\beta$ is a refinement of $\alpha$ (that is if each element of $\beta$ is a subset of one or more elements of $\alpha$ ) while $\alpha$ is not a refinement of $\beta$.

Let $\beta>\alpha$. We get by definition a projection $\sigma_{\alpha}^{\beta}$ of $N_{\beta}$ into $N_{\alpha}$ if we assign to each vertex $e_{\beta j}$ of $N_{\beta}$ any vertex $e_{\alpha i}$ of $N_{\alpha}$ satisfying the condition that $o_{\beta j} \subseteq o_{\alpha i}$. It is easily seen that the nerves $N_{\alpha}$ with their special subcomplexes $C_{\alpha}$ and projections $\sigma_{\alpha}^{\beta}$ constitute a simplicial spectrum $\left\{N_{\alpha}, C_{\alpha}, \sigma_{\alpha}^{\beta}\right\}$ called the simplicial spectrum of the locally bicompact space $\mathbf{K}$. According to section 29 this simplicial spectrum generates the cell spectrum

$$
K=\left\{K_{\alpha}, \varpi_{\alpha}^{\beta}\right\}
$$

called the cell spectrum of the locally bicompact space $\mathbf{K}$. The Betti groups

$$
\Delta_{K}^{r}=\lim \inf \left(\Delta_{K_{\alpha}}^{r}, \varpi_{\alpha}^{\beta}\right) ; \quad \nabla_{K}^{r}=\lim \sup \left(\nabla_{K_{\alpha}}^{r}, \pi_{\beta}^{\alpha}\right)
$$

of the cell spectrum $K$ are called Betti groups of the space $\mathbf{K}$ and are denoted correspondingly by $\Delta_{\mathbf{K}}^{r}, \nabla_{\mathbf{K}}^{r}$.

If the space $\mathbf{K}$ is bicompact, then so is its spectrum $K$ and the previous definitions are simplified by $C_{\alpha}=0, K_{\alpha}=N_{\alpha}$.

31. The figures $\mathbf{K}, \mathbf{A}, \boldsymbol{\Gamma}$ and $K, A, G$. Let the notations of the previous section hold. Let $\mathbf{A}$ be a closed set in the locally bicompact normal space $\mathbf{K}$; the complement $\mathbf{\Gamma}=\mathbf{K} \backslash \mathbf{A}$ is open.

Denote by $A_{\alpha} \subseteq K_{\alpha}$ the closed subcomplex of all simplices $T_{\alpha} \in K_{\alpha}$ satisfying the condition: the associate set $o_{\alpha i} \in \alpha$ of each vertex $e_{\alpha i}$ of $T_{\alpha}$ has points in common with A. It is easy to see that the projections $\sigma_{\alpha}^{\beta}$ of $N_{\beta}$ into $N_{\alpha}$ defined in the last section satisfy the conditions of the Corollary 28.2 and thus define in addition to the cell spectrum (30) the cell spectra

$$
A=\left\{A_{\alpha}, \varpi_{\alpha 0}^{\beta 0}\right\}
$$

(5) Our terminology is correct, each nerve being, for example, the nerve of the system of the open stars of its own vertices. 
and

$$
G=\left\{G_{\alpha}, \underset{\varpi_{\alpha 1}^{\beta 1}}{\beta}\right\}
$$

Now $\Delta_{\mathbf{K}}^{r}, \nabla_{\mathbf{K}}^{r}$ are by definition identical with $\Delta_{K}^{r}, \nabla_{K}^{r}$ while, as we shall see in the next paragraph, there exist certain natural isomorphisms between the groups

$$
\begin{array}{rrrrr}
\Delta_{A}^{r} & \text { and } & \Delta_{\mathbf{A} ;}^{r} & \nabla_{A}^{r} & \text { and } \nabla_{\mathbf{A}}^{r}, \\
\Delta_{G}^{r} & \text { and } & \Delta_{\boldsymbol{\Gamma}}^{r} ; & \nabla_{G}^{r} & \text { and } \nabla_{\boldsymbol{\Gamma}}^{r} .
\end{array}
$$

These isomorphisms transform the homomorphisms $E_{K}^{A}, E_{K}^{G}, J_{A}^{K}, J_{G}^{K}$ into the homomorphisms $E_{\mathbf{K}}^{\mathbf{A}}, E_{\mathbf{K}}^{\mathbf{\Gamma}}, J_{\mathbf{A}}^{\mathbf{K}}, J_{\mathbf{\Gamma}}^{\mathbf{K}}$ and thus lead to the definition of the groups of the figure $\mathbf{K}, \mathbf{A}, \mathbf{\Gamma}$.

\section{§9. ThE NATURAL ISOMORPHISMS, THE GROUPS AND THE HOMOMOR PHISMS OF THE FIGURE $\mathbf{K}, \mathbf{A}, \boldsymbol{\Gamma}$}

32. Special types of coverings. Throughout this chapter $\mathbf{K}$ means always a normal locally bicompact space; by a covering (without any special attribute) is always meant a finite covering by open sets (of the given space).

In any covering $\alpha=\left\{o_{\alpha 1}, \cdots, o_{\alpha s}\right\}$ of $\mathbf{K}$ we denote by $o_{\alpha 1}, \cdots, o_{\alpha p}$ and call elements of the first kind the elements meeting $\mathbf{A}$; the remaining elements $o_{\alpha, p+1}, \cdots, o_{\alpha s}$ have no points in common with $\mathbf{A}$ and are called elements of the second kind. An element of the second kind is called a boundary or an inner element according to whether its closure has or has not points in common with $\mathbf{A}$.

A covering $\alpha=\left\{o_{\alpha 1}, \cdots, o_{\alpha p}, \cdots, o_{\alpha s}\right)$ of $\mathbf{K}$ is called cogredient with $\mathbf{A}$ if it satisfies the following conditions:

1. Any two among the point sets $\mathbf{A} \cap o_{i}, i \leqq p$, are different.

2. If $i_{0}, \cdots, i_{r} \leqq p$ and $o_{\alpha i_{0}} \cap \cdots \cap o_{\alpha i_{r}} \neq 0$, then $\mathbf{A} \cap o_{\alpha i_{0}} \cap \cdots \cap o_{\alpha i_{r}} \neq 0$.

3. The bicompactness of $\overline{\mathbf{A} \cap o_{\alpha i}}, i \leqq p$, implies that of $\bar{o}_{\alpha i}$.

Let $\alpha=\left\{o_{\alpha 1}, \cdots, o_{\alpha p}, \cdots, o_{a s}\right\}$ be a covering of $\mathbf{K}$. Denote by $\phi$ the sum of all those sets $\bar{o}_{\alpha i}$ which are bicompact and lie in $\boldsymbol{\Gamma}$. The covering $\alpha$ is called cogredient with $\Gamma$, if it satisfies the following conditions:

1. The elements of the second kind of $\alpha$ form a covering of $\Gamma$ (denoted by $\Gamma \alpha$ ).

2. No element of the first kind meets $\phi$.

REMARK. In my paper [1] coverings cogredient with $\Gamma$ were called "regular with respect to $\Gamma$."

The covering $\alpha=\left\{o_{\alpha 1}, \cdots, o_{\alpha p}, \cdots, o_{\alpha s}\right\}$ is called regular ("regular with respect to $\mathbf{A}$ " in the terminology of [1]) if the following conditions are fulfilled:

1. The covering $\alpha$ contains no boundary element of the second kind.

2. If $\bar{o}_{\alpha i_{0}} \cap \cdots \cap \bar{o}_{\alpha i_{r}} \neq 0$ and $i_{0}, \cdots, i_{r} \leqq p$, then $\mathbf{A} \cap o_{\alpha i_{0}} \cap \cdots \cap o_{\alpha i_{r}} \neq 0$. 
To finish with these preliminary notions take in $K_{\alpha}$ all simplices $T_{\alpha}$ with the following property: Each vertex of $T_{\alpha}$ corresponds either to an element $o_{\alpha i} \in \alpha$ of the first kind or to a boundary element of the second kind; these simplices form a closed subcomplex $A_{a}^{\prime}$ of $K_{\alpha}$ and for $\beta>\alpha$ the complex $A_{\beta}^{\prime}$ is mapped by any $\sigma_{\alpha}^{\beta}$ in to $A_{\alpha}^{\prime}$, the conditions of Corollary 28.2 being satisfied. Therefore we have the cell spectrum $A^{\prime}=\left\{A_{\alpha}^{\prime}, \varpi_{\alpha 0}^{\prime \beta 0}\right\}$ and the still more important for us cell spectrum

$$
G^{\prime}=\left\{G_{\alpha}^{\prime}, \varpi_{\alpha 1}^{\prime \beta 1}\right\},
$$

where $G_{\alpha}^{\prime}=K_{\alpha} \backslash A_{\alpha}^{\prime}$, and $\varpi_{\alpha 0}^{\prime \beta 0}, \varpi_{\alpha 0}^{\prime \beta 1}$ are the projections of $A_{\beta}^{\prime}, G_{\beta}^{\prime}$ in to $A_{\alpha}^{\prime}, G_{\alpha}^{\prime}$ generated by $\sigma_{\alpha}^{\beta}$.

32.2. Obviously if $\alpha$ is regular, then $A_{\alpha}^{\prime}=A_{\alpha}, G_{\alpha}^{\prime}=G_{\alpha}$.

The following results are proved in detail in my paper [1] (one can read the proofs without reading the rest of the paper):

32.3. Any covering of $\mathbf{K}$ has a regular refinement [1, p. 80, Theorem 6.22].

32.4. Any covering of $\mathbf{K}$ has a refinement cogredient with $\boldsymbol{\Gamma}$-and even more:

32.5. Any two coverings $\alpha$ and $\beta$ of $\mathbf{K}$ have such a common refinement $\gamma$ cogredient with $\boldsymbol{\Gamma}$ that every element of the second kind of $\gamma$ is contained in an element of the second kind of $\alpha$ and in an element of the second kind of $\beta[1$, p. 89, Lemma 8.5].

32.6. For every covering $\alpha \boldsymbol{\Gamma}$ of $\Gamma$ there is a covering $\alpha$ of $\mathbf{K}$ cogredient with $\Gamma$ and such that $\alpha_{\Gamma}=\Gamma \alpha[1$, pp. 87-88, Theorem 8.33].

Let us prove now:

32.7. Every covering $\alpha=\left\{o_{\alpha 1}, \cdots, o_{\alpha p}, \cdots, o_{\alpha 8}\right\}$ of $\mathbf{K}$ has a refinement cogredient with $\mathbf{A}$.

Proof. Let

$$
\mathbf{A} \cap o_{\alpha 1}, \cdots, \mathbf{A} \cap o_{\alpha q}, \quad q \leqq p,
$$

be chosen among the $\mathbf{A} \cap o_{c 1}, \cdots, \mathbf{A} \cap o_{\alpha p}$ in such a manner that no proper subsystem of the system (32.71) is a covering of $\mathbf{A}$. Take such closed sets $a_{i} \subseteq\left(\mathbf{A} \cap o_{\alpha i}\right), i=1,2, \cdots, q$, as to get a closed covering of $\mathbf{A}$. Choose for each $a_{i}$ a neighborhood $O a_{i} \subseteq o_{\alpha i}$ under the following conditions:

1. All $O a_{i}$ are different.

2. Any $O a_{i_{0}}, \cdots, O a_{i_{r}}$ have points in common only if $a_{i_{0}}, \cdots, a_{i_{r}}$ have.

3. The bicompactness of $\bar{a}_{i}$ implies that of $\overline{O a_{i}}$. The sum of $O a_{1}, \cdots, O a_{q}$ is a neighborhood $O \mathbf{A}$ of $\mathbf{A}$. Take the closed sets $b_{h} \subset \mathbf{K} \backslash O \mathbf{A}$ so as to get a closed covering $\left\{b_{1}, \cdots, b_{u}\right\}$ of $\mathbf{K} \backslash O \mathbf{A}$ in such a way that each of the $b_{h}$ is contained in some $o_{\alpha i}$. Then define $O b_{h}$ as $o_{\alpha i} \cap(\mathbf{K} \backslash \mathbf{A})$, where $o_{\alpha i}$ is any element of $\alpha$ containing $b_{h}$. The covering $O a_{1}, \cdots, O a_{q}, O b_{1}, \cdots, O b_{u}$ has the required properties.

Let $\alpha_{\mathbf{A}}$ be a covering of $\mathbf{A}$. We write $\alpha_{\mathbf{A}}=\mathbf{A} \alpha, \alpha$ being a covering of $\boldsymbol{K}$, if the elements of $\alpha_{\mathbf{A}}$ are in (1-1)-correspondence with the elements of the 
first kind of $\alpha$, each element of $\alpha_{\mathbf{A}}$ being the intersection of $\mathbf{A}$ with the corresponding element of $\alpha$. From 32.7 follows:

32.8. If we consider among the coverings of $\mathbf{A}$ only those of the form $\alpha_{A}=\mathbf{A} \alpha$, where $\alpha$ is a covering of $\mathbf{K}$ cogredient with $A$, and set for these coverings $\mathbf{A} \beta>\mathbf{A} \alpha$ only if $\beta>\alpha$ we get a cofinal part of the set of all coverings of $\mathbf{A}$.

33. A lemma on group spectra.

LEMMA 33. Let the group spectra:

(inverse) $\left\{\bar{U}_{\alpha \lambda}, \varpi_{\alpha \lambda}^{\beta \mu}\right\}$ and $\left\{\bar{U}_{\alpha}, \varpi_{\alpha}^{\beta}\right\} ;($ direct $)\left\{U_{\alpha \lambda}, \pi_{\beta \mu}^{\alpha \lambda}\right\}$ and $\left\{U_{\alpha}, \pi_{\beta}^{\alpha}\right\}$

satisfy the following conditions:

1. There exists an isomorphism $\phi_{\alpha}^{\alpha \lambda}$

$$
\text { of } \bar{U}_{\alpha \lambda} \text { onto } \bar{U}_{\alpha} ; \text { of } U_{\alpha \lambda} \text { onto } U_{\alpha} \text {. }
$$

2. $\beta \mu>\alpha \lambda$ implies $\beta>\alpha$.

3. For every

we have

$$
\bar{u}_{\beta \mu} \in \bar{U}_{\beta \mu} ; \quad u_{\alpha \lambda} \in U_{\alpha \lambda}
$$

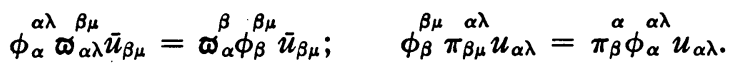

Under these hypotheses if we replace the elements of

$$
\text { each thread }\left\{\bar{u}_{\alpha \lambda}\right\} \text {; each bundle }\left\{u_{\alpha \lambda}\right\}
$$

by their images under $\phi_{\alpha}^{\alpha \lambda}$ we get an isomorphism between

$\lim \inf \left(\bar{U}_{\alpha \lambda}, \varpi_{\alpha \lambda}^{\beta \mu}\right)$ and $\lim \inf \left(\bar{U}_{\alpha}, \varpi_{\alpha}^{\beta}\right) ; \lim \sup \left(U_{\alpha \lambda}, \pi_{\beta \mu}^{\alpha \lambda}\right)$ and $\lim \sup \left(U_{\alpha}, \pi_{\beta}^{\alpha}\right)$.

The proof is easy and may be found in $[1$, p. 62, Theorem 3.61].

34. The natural isomorphisms between $\Delta_{A}^{r}, \nabla_{A}^{r}$ and $\Delta_{\mathbf{A}}^{r}, \nabla_{\mathbf{A}}^{r}$. We return to the figures of section 31 but we change slightly our notations: we denote now by $\alpha$ any covering of $\mathbf{A}$ which can be written in the form $\alpha=\mathbf{A} \alpha \lambda$, where $\alpha \lambda$ is a covering of $\mathbf{K}$ cogredient with $\mathbf{A}$ (and satisfying the condition $\mathbf{A} \alpha \lambda=\alpha$ ). We use $N_{\alpha \lambda}, N_{\alpha}$ for the nerves of $\alpha \lambda$ and $\alpha$, write $\sigma_{\alpha \lambda}^{\beta \mu}$ for the projections of $N_{\beta \mu}$ into $N_{\alpha \lambda}$, while $\sigma_{\alpha}^{\beta}$ denote now only those projections of $N_{\beta}$ into $N_{\alpha}$ which are generated by a projection $\sigma_{\alpha \lambda}^{\beta \mu}$. As usual $K_{\alpha \lambda}, K_{\alpha}$ is the subcomplex of $N_{\alpha \lambda}, N_{\alpha}$ respectively formed by all simplices which have among their vertices at least one corresponding to an element of $\alpha \lambda$ (of $\alpha$ ) with bicompact closure; $A_{\alpha \lambda}$ is the subcomplex of $K_{\alpha \lambda}$ defined by the vertices which correspond to elements of $\alpha \lambda$ meeting $\mathbf{A}$.

The passage from $\alpha \lambda$ to $\alpha=\mathbf{A} \alpha \lambda$ produces an isomorphic mapping $\left({ }^{6}\right)$ of

${ }^{(6)}$ Let $N, N^{\prime}$ be nerves, $Q, Q^{\prime}$ subcomplexes of $N, N^{\prime}$ respectively. We define $[Q] \subseteq N$ as the nerve consisting of all simplices of $Q$ and of all faces of these simplices; the same for $\left[Q^{\prime}\right]$. Any (1-1)-mapping of $Q$ on $Q^{\prime}$ generated by a (1-1)-simplicial mapping of $[Q]$ on $\left[Q^{\prime}\right]$ is called isomorphic. 
$A_{\alpha \lambda}$ on to $K_{\alpha}$ and therefore an isomorphism $\phi_{\alpha}^{\alpha \lambda}$ of the groups $\Delta_{\alpha \lambda 0}^{r}, \nabla_{\alpha \lambda 0}^{r}$ (of $A_{\alpha \lambda}$ ) on the groups $\Delta_{\alpha}^{r}, \nabla_{\alpha}^{r}$ (of $K_{\alpha}$ ). The isomorphism $\phi_{\alpha}^{\alpha \lambda}$ satisfies the hypotheses of Lemma 33 and thus generates a well defined isomorphism

$$
\begin{aligned}
& \text { of } \lim \inf \left(\Delta_{\alpha \lambda 0}^{r}, \varpi_{\alpha \lambda 0}^{\beta \mu 0}\right) \text { on } \lim \inf \left(\Delta_{\alpha}^{r}, \varpi_{\alpha}^{\beta}\right) \text {; } \\
& \text { of } \lim \sup \left(\nabla_{\alpha \lambda 0}^{r}, \pi_{\beta \mu 0}^{\alpha \lambda 0}\right) \text { on } \lim \sup \left(\nabla_{\alpha}^{r}, \pi_{\beta}^{\alpha}\right) \text {. }
\end{aligned}
$$

Since the coverings $\alpha \lambda$ form a cofinal part of the set of all coverings of $\boldsymbol{K}$ while the coverings $\alpha$ form a cofinal part of the set of all coverings of $\mathbf{A}$ we may identify

$$
\begin{array}{ll}
\lim \inf \left(\Delta_{\alpha \lambda 0}^{r}, \varpi_{\alpha \lambda 0}^{\beta \mu 0}\right) \text { with } \Delta_{A}^{r} ; & \lim \sup \left(\nabla_{\alpha \lambda 0}^{r}, \pi_{\beta \mu 0}^{\alpha \lambda 0}\right) \text { with } \nabla_{A}^{r}, \\
\lim \inf \left(\Delta_{\alpha}^{r}, \varpi_{\alpha}^{\beta}\right) \text { with } \Delta_{\mathbf{A}}^{r} ; & \lim \sup \left(\nabla_{\alpha}^{r}, \pi_{\beta}^{\alpha}\right) \text { with } \nabla_{\mathbf{A}}^{r}
\end{array}
$$

and get in this way the natural isomorphism $(A \mathbf{A})$ of $\Delta_{A}^{r}, \nabla_{A}^{r}$ on $\Delta_{A}^{r}, \nabla_{A}^{r}$; the inverse isomorphism is denoted by $(\mathbf{A} A)$; we return to them in section 37 .

35. The natural isomorphism between $\Delta_{\Gamma}^{r}, \nabla_{\Gamma}^{r}$ and $\Delta_{G}^{r}, \nabla_{G}^{r}$. Now we denote by $\alpha$ any covering of $\Gamma$, by $\alpha \lambda$ an arbitrary covering of $\boldsymbol{K}$ cogredient with $\Gamma$ and such that $\Gamma \alpha \lambda=\alpha$. Then $N_{\alpha \lambda}, N_{\alpha}, K_{\alpha \lambda}, K_{\alpha}$ have an obvious meaning. We retain among the inequalities $\beta \mu>\alpha \lambda$ only those for which there exists a projection $\sigma_{\alpha \lambda}^{\beta \mu}$ which assigns to each element $o_{\beta \mu} \in \beta \mu$ of the second kind an element $o_{\alpha \lambda} \in \alpha \lambda, o_{\alpha \lambda} \subseteq o_{\beta \mu}$, of the second kind. Only these $\sigma_{\alpha \lambda}^{\beta \mu}$ will be taken into account. In this way we get by virtue of 32.5 a cofinal part

$$
K_{1}=\left\{K_{\alpha \lambda}, \varpi_{\alpha \lambda}^{\beta \mu}\right\}
$$

of the spectrum $K$.

According to section 32 we denote by $G_{\alpha \lambda}^{\prime}$ the open subcomplex of $K_{\alpha \lambda}$, the simplices $T_{\alpha \lambda}^{\prime} \in G_{\alpha \lambda}^{\prime}$ being defined by the following property: among the vertices of $T_{\alpha \lambda}$ there is at least one corresponding to an inner element (of the second kind) of the covering $\alpha \lambda$. We denote by $\widetilde{\omega}_{\alpha \lambda}^{\prime \beta \mu}$ the projection of the cell complex $G_{\beta \mu}^{\prime}$ into the cell complex $G_{\alpha \lambda}^{\prime}$ generated by $\sigma_{\alpha \lambda}^{\beta \mu}$. It is easily seen $[1$, p. 88 , formula $(8.41)]$ that $K_{\alpha}=G_{\alpha \lambda}^{\prime}$ for every $\alpha \lambda$; thus we may identify the group

$$
\Delta_{\alpha}^{r}=\Delta_{K_{\alpha}}^{r} \text { with } \Delta_{(\alpha \lambda)^{\prime}}^{r}=\Delta_{G_{\alpha \lambda}^{\prime}}^{r} ; \quad \nabla_{\alpha}^{r}=\nabla_{K_{\alpha}}^{r} \text { with } \nabla_{(\alpha \lambda)^{\prime}}^{r}=\nabla_{G_{\alpha \lambda}^{\prime}}^{r}
$$

and therefore

$$
\begin{aligned}
& \Delta_{\Gamma}^{r}=\lim \inf \left(\Delta_{\alpha}^{r}, \varpi_{\alpha}^{\beta}\right) \text { with } \Delta_{G^{\prime}}^{r}=\lim \inf \left(\Delta_{(\alpha \lambda)^{\prime}}^{r}, \boldsymbol{\Phi}_{\alpha \lambda}^{\prime \beta \mu}\right), \\
& \nabla_{\Gamma}^{r}=\lim \sup \left(\nabla_{\alpha}^{r}, \pi_{\beta}^{\alpha}\right) \text { with } \nabla_{G^{\prime}}^{r}=\lim \sup \left(\nabla_{(\alpha \lambda)^{\prime},}^{r}, \pi_{\beta \mu}^{\alpha \lambda}\right) .
\end{aligned}
$$

But since the coverings $\alpha \lambda$ (cogredient with $\Gamma$ ) on the one hand and the regular coverings on the other form both cofinal parts of the partially ordered set 
of all coverings of $\mathbf{K}$ we get (taking into account 32.2) a natural isomorphism between

$$
\Delta_{G^{\prime}}^{r} \text { and } \Delta_{G}^{r} ; \quad \nabla_{G^{\prime}}^{r} \text { and } \nabla_{G}^{r} \text {, }
$$

and therefore between

$$
\Delta_{\Gamma}^{r} \text { and } \Delta_{G}^{r} ; \quad \nabla_{\Gamma}^{r} \text { and } \nabla_{G}^{r} \text {. }
$$

This isomorphism (considered from $\Gamma$ to $G$ ) is denoted by $(\boldsymbol{\Gamma} G$ ), the inverse isomorphism by $(G \Gamma)$.

36. The groups of the figure $K, A, \Gamma$ and the results concerning them. Because of the identities $\Delta_{\mathbf{K}}^{r}=\Delta_{K}^{r}, \nabla_{\mathbf{K}}^{r}=\nabla_{K}^{r}$ the natural isomorphisms transform the homomorphisms

$$
E_{K}^{A}, \quad E_{K}^{G}, \quad J_{A}^{K}, \quad J_{G}^{K}
$$

(of the groups $\Delta_{A}^{r}, \nabla_{G}^{r}, \nabla_{K}^{r}, \Delta_{K}^{r}$ into the groups $\Delta_{K}^{r}, \nabla_{K}^{r}, \nabla_{A}^{r}, \Delta_{G}^{r}$ ) into the homomorphisms

$$
E_{\mathbf{K}}^{\mathbf{A}}, \quad E_{\mathbf{K}}^{\mathbf{\Gamma}}, \quad E_{\mathbf{A}}^{\mathbf{K}}, \quad J_{\mathbf{\Gamma}}^{\mathbf{K}}
$$

of the groups

$$
\Delta_{\mathrm{A}}^{r}, \quad \nabla^{r} \boldsymbol{\Gamma}, \quad \nabla_{\mathbf{K}}^{r}, \quad \Delta_{\mathbf{K}}^{r}
$$

in to the groups

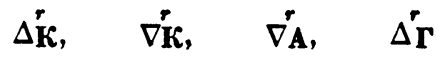

and thus define the groups

$$
\begin{aligned}
& \Delta_{\mathbf{K} \mathbf{A}}^{r}=E_{\mathbf{K}}^{\mathbf{A}} \Delta_{\mathbf{A}}^{r}=E_{K}^{A}(\mathbf{A} A) \Delta_{\mathbf{A}}^{r}=E_{K}^{A} \Delta_{A}^{r}=\Delta_{K A}^{r} ; \quad \nabla^{r} \mathbf{K}=E_{\mathbf{K}}^{\mathbf{r}} \nabla_{\mathbf{\Gamma}}^{r}=\nabla_{K G}^{r}, \\
& \Delta \Delta_{\mathbf{A} K}^{r}=J_{\mathbf{A} \nabla_{K}^{\mathbf{K}}}^{\mathbf{K}}=\left(A_{\mathbf{A}}\right) J_{A}^{K} \nabla_{K}^{r}=\left(A_{\mathbf{A}}\right) \nabla_{A K}^{r} ; \quad \Delta_{\mathbf{\Gamma} K}^{r}=J_{\mathbf{\Gamma}}^{\mathbf{K}} \Delta_{\mathbf{K}}^{r}=(G \mathbf{\Gamma}) \Delta_{G K}^{r} \text {, } \\
& \Delta_{\mathbf{A}}^{r}: \mathbf{K}=\left(E_{\mathbf{K}}^{\mathbf{A}}\right)^{-1} 0 \mathbf{K}=(A \mathbf{A})\left(E_{K}^{A}\right)^{-1} 0_{K}=(A \mathbf{A}) \Delta_{A: K}^{r} ; \nabla^{r} \mathbf{\Gamma}: \mathbf{K}=(E \mathbf{K})^{-1} 0 \mathbf{K}=(G \mathbf{\Gamma}) \nabla_{G: K}^{r}, \\
& \nabla_{\mathbf{A}}^{r}: \mathbf{K}=\nabla_{\mathbf{A}}^{r}-\nabla_{\mathbf{A} K}^{r}=(A \mathbf{A}) \nabla_{A}^{r}-(A \mathbf{A}) \nabla_{A K}^{r}=(A \mathbf{A}) \nabla_{A: K}^{r} ; \Delta r \mathbf{r}: \mathbf{K}(G \mathbf{\Gamma}) \Delta_{G: K}^{r} .
\end{aligned}
$$

If we define the product of an element of $\Delta_{\mathbf{A}}^{r}$ (of $\nabla_{\Gamma}^{r}$ ) with an element of $\nabla_{\mathbf{A}}^{r}$ (of $\Delta_{\Gamma}^{r}$ ) as the product of the corresponding elements of $\Delta_{A}^{r}, \nabla_{A}^{r}$ (of $\nabla_{G}^{r}, \Delta_{G}^{r}$ ), we see that not only all isomorphisms but also all dualities and annihilations between the groups of the figure $K, A, G$ are transformed by the natural isomorphisms into the corresponding relations between the groups of the figure $\mathbf{K}, \mathbf{A}, \boldsymbol{\Gamma}$. Therefore all results (14.1)-(14.3') formulated in section 14 for the figure $K, A, G$ hold for $\mathbf{K}, \mathbf{A}, \mathbf{\Gamma}$.

37. Remarks on the natural isomorphisms and the groups of the figure $\mathbf{K}, \mathbf{A}, \boldsymbol{\Gamma}$. Let an element of the group $\nabla_{A}^{r}$ (of the group $\Delta_{A}^{r}$ ) be given. Take only the coverings $\alpha \lambda$ of $\mathbf{K}$ cogredient with $\mathbf{A}$. Using the subscript $\alpha \lambda 0$ for $\nabla$-cycles ( $\Delta$-classes) on $A_{\alpha \lambda}$ we can write for any $z^{r} \in \nabla_{A}^{r}, \bar{z}^{r} \in \Delta_{A}^{r}$ : 


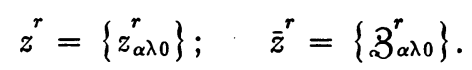

As we know $A_{\alpha \lambda}$ can be identified with the nerve of $\alpha=\mathbf{A} \alpha \lambda$; therefore $z^{r}, \bar{z}^{r}$ can be considered as elements of $\nabla_{\mathbf{A}}^{r}, \Delta_{\mathbf{A}}^{r}$ and this identification represents an isomorphism-our natural isomorphism- $(A \mathbf{A})$.

For a moment, let us denote by $\alpha$ coverings of $\boldsymbol{\Gamma}$; let $z^{r}=\left\{z_{\alpha}^{r}\right\} \in \nabla_{\boldsymbol{\Gamma}}^{r}$. Each $z_{\alpha}^{\prime}$ can be considered as a $\nabla$-cycle on $G_{\alpha \lambda}^{\prime}$, thus $\left(G_{\alpha \lambda}^{\prime} \subseteq G_{\alpha \lambda}\right.$ being open on $G_{\alpha \lambda}$ ) as a $\nabla$-cycle on $G_{\alpha \lambda}$, where $\alpha \lambda$ is cogredient with $\Gamma$ and $\alpha=\Gamma \alpha \lambda$. Conserve among the coverings of $\boldsymbol{K}$ only those which are cogredient with $\boldsymbol{\Gamma}$ and among the projections $\sigma_{\alpha \lambda}^{\beta \mu}$ of these coverings only projections which map elements of the second kind of $\beta \mu$ on elements of the second kind of $\alpha \lambda$. Then we can identify our $z^{r}$ with a certain element of $\nabla_{G}^{r}$ and this identification is the natural isomorphism $(\boldsymbol{\Gamma} G)$. We needed the regular coverings only to prove that this isomorphism maps $\nabla_{\Gamma}^{r}$ on to $\nabla_{G}^{r}$.

Now let $\alpha$ be a covering of $\mathbf{K}$. Let

$$
\bar{z}_{G}^{r}=\left\{乃_{\alpha 1}^{r}\right\} \in \Delta_{G}^{r} .
$$

Assign to any $\bar{z}_{\alpha 1}^{r} \in \mathcal{B}_{\alpha 1}^{r}$ the cycle

$$
\bar{z}_{(\alpha)^{\prime}}^{r}=J_{G_{\alpha}^{\prime} \bar{z}_{\alpha 1}^{r}}^{G}
$$

(this is a customary method if we consider $\bar{z}_{\alpha 1}^{r}$ as a Lefschetz relative cycle $\bmod A_{\alpha}$ and keep in mind that $\left.A_{\alpha}^{\prime} \supseteq A_{\alpha}\right)$. We get in this way an isomorphism of $\Delta_{G}^{\tau}$ on $\Delta_{G^{\prime}}^{r}$ (the "on" is again proved using the regular coverings). If now we take in to account only the coverings of $\mathbf{K}$ cogredient with $\boldsymbol{\Gamma}$ we can identify $\bar{z}_{G^{\prime}}^{r}=\left\{\bar{z}_{(\alpha)^{\prime}}^{r}\right\} \in \Delta_{G^{\prime}}^{r}$ with a certain element of $\Delta_{\Gamma}^{r}$. Thus by the transition from $\Delta_{G}^{r}$ to $\Delta_{G^{\prime}}^{r}$ and then to $\Delta_{\boldsymbol{\Gamma}}^{r}$ we get the natural isomorphism $(G \boldsymbol{\Gamma})$ of $\Delta_{G}^{r}$ on to $\Delta_{\Gamma}^{r}$.

To the simple intuitive meaning of the natural isomorphisms corresponds a direct definition of the groups of the figure $\mathbf{K}, \mathbf{A}, \boldsymbol{\Gamma}$. Since $\Delta_{\mathbf{K A}}^{r}=\Delta_{K A}^{r}$, $\nabla_{\mathbf{K} \Gamma}^{r}=\nabla_{K G}^{r}, \nabla_{\mathbf{A}: \mathbf{K}}^{r}=\nabla_{\mathbf{A}}^{r}-\nabla_{\mathbf{A K}}^{r}, \Delta_{\mathbf{\Gamma}: \mathbf{K}}^{r}=\Delta_{\boldsymbol{\Gamma}}^{r}-\Delta_{\mathbf{\Gamma K}}^{r}$, we are interested in the groups $\nabla_{\mathbf{A K}}^{r}, \Delta_{\mathbf{\Gamma K}}^{r} ; \Delta_{\mathbf{A}: \mathbf{K}}^{r}, \nabla_{\mathbf{\Gamma}: \mathbf{K}}^{r}$ only.

The elements of the bundle $z^{r} \in \nabla_{A}^{r}$ being identified with the $\nabla$-cycles $z_{\alpha \lambda 0}^{r}$ on $A_{\alpha \lambda}, \alpha \lambda$ cogredient with $\mathbf{A}$, the bundles $z^{r}=\left\{z_{\alpha \lambda 0}^{r}\right\}$ belonging to $\nabla_{\mathbf{A K}}^{r}$ are characterized by the property: there is for each $z_{\alpha \lambda_{0}}^{r} \in z^{r}$ a $\beta \mu>\alpha \lambda$ such that $\pi_{\beta \mu 0}^{\alpha \lambda 0} z_{\alpha \lambda 0}^{r}$ is extensible over $K_{\beta \mu}$.

The elements $\bar{z}^{r} \in \Delta_{\text {TK }}^{r}$ are characterized in the following way: we first identify the elements of the thread $\bar{z}^{r}$ with $\Delta$-classes of $G_{\alpha \lambda}^{\prime}(\alpha \lambda$ cogredient with $\Gamma$ ) and thus identify the thread $\bar{z}^{r}$ with a certain thread $\bar{z}^{\prime r}$ of the spectrum $G^{\prime}$; then using regular coverings we identify the thread $\bar{z}^{\prime r}$ with a thread of the spectrum $G$; this latter must be extensible in the sense of section 26 .

The elements of $\Delta_{\mathbf{A}: \mathbf{K}}^{r}$ are threads $\bar{z}^{r} \in \Delta_{\mathbf{A}}^{r}$ bounding on $\mathbf{K}$; it means that if we consider $\bar{z}_{\alpha}^{r} \in \mathcal{B}_{\alpha}^{r} \in \bar{z}^{r} \in \Delta_{\mathbf{A}: \mathbf{K}}^{r}$ as a $\Delta$-cycle on $A_{\alpha \lambda}(\alpha \lambda$ cogredient with $\mathbf{A}$, $\mathbf{A} \alpha \lambda=\alpha$ ), this cycle bounds on $K_{\alpha \lambda}$. 
The elements of $\nabla_{\boldsymbol{\Gamma}: \boldsymbol{K}}^{r}$ are bundles $z^{r} \in \nabla_{\boldsymbol{\Gamma}}^{r}$ bounding on $\mathbf{K}$; it means that if we consider the elements of the bundle $z^{r}$ as cycles on $G_{\alpha \lambda}$ ( $\alpha \lambda$ cogredient with $\Gamma$ ), these cycles are elements of the bundle representing the zero of $\nabla_{K}^{r}$. In other words, if $z_{\alpha \lambda}^{r} \in z^{r}$ and $z^{r} \in \nabla_{\Gamma}^{r}$, then there exists a $\beta \mu>\alpha \lambda$ such that $\pi_{\beta \mu}^{\alpha \lambda} z_{\alpha \lambda}^{r}$ bounds on $K_{\beta \mu}$.

As a matter of fact, the elements of the groups $\nabla_{\mathbf{A}: \mathbf{K}}^{r}=\nabla_{\mathbf{A}}^{r}-\nabla_{\mathbf{A K}}^{r}$ can be defined as extension classes of $\nabla$-cycles: let $\alpha, \beta$ be two coverings of $A$; define $N_{\alpha}, N_{\beta}, K_{\alpha}, K_{\beta}$ as in section 34 ; two $\nabla$-cycles $z_{\alpha}^{r}, z_{\beta}^{r}$ on $K_{\alpha}, K_{\beta}$ respectively belong by definition to the same extension class if there exist coverings $\alpha \lambda, \beta \mu$, $\gamma \nu$ cogredient with $\mathbf{A}$ such that $\alpha=\mathbf{A} \alpha \lambda, \beta=\mathbf{A} \beta \mu, \gamma \nu>\alpha \lambda, \beta \mu$ and $\pi_{\gamma \nu 0}^{\alpha \lambda 0} z_{\alpha}^{z}$ $-\pi_{\gamma \nu 0}^{\beta \mu 0} z_{\beta}^{\gamma}$ be a $\nabla$-cycle on $A_{\gamma \nu}$, extensible over $K_{\gamma \nu}$.

The elements of $\Delta_{\Gamma: K}^{r}$ could be defined as extension classes of threads: two threads $\bar{z}^{r}=\left\{B_{\alpha}^{r}\right\} \in \Delta_{\Gamma}^{r}$ and $\bar{z}^{\prime r}=\left\{B_{\alpha}^{\prime r}\right\} \in \Delta_{\Gamma}^{r}$ belong by definition to the same extension class if for each $\alpha$ the cycle $\bar{z}_{\alpha}^{r}-\bar{z}_{\alpha}^{\prime r}$ (where $\bar{z}_{\alpha}^{r} \in \mathfrak{Z}_{\alpha}^{r}, \bar{z}_{\alpha}^{\prime r} \in \mathfrak{Z}_{\alpha}^{\prime r}$ ) considered as a cycle on $G_{\alpha \lambda}^{\prime}(\alpha \lambda$ cogredient with $\Gamma$ and $\Gamma \alpha \lambda=\alpha)$ is extensible over $K_{\alpha \lambda}$.

38. Another form of the definition of the natural isomorphism (A $A$ ). This other form will be used in Chapter IV, remark 1 in section 43 . We conserve the notations of sections 30 and 31 and suppose for the sake of simplicity that $\mathbf{K}$ is bicompact. Take in each $K_{\alpha}$ the closed subcomplex $A_{\alpha}^{\prime \prime}$ with the same vertices as $A_{\alpha}$ and simplices $\left|e_{i_{0}} \cdots e_{i_{r}}\right|$ corresponding to those elements $o_{i_{0}}, \cdots, o_{i_{r}}$ of the covering $\alpha$ of $\mathbf{K}$ which satisfy the condition

$$
\mathbf{A} \cap o_{i_{0}} \cap \cdots \cap o_{i_{r}} \neq 0 .
$$

Obviously $A_{\alpha}^{\prime \prime}$ is a subcomplex of $A_{\alpha}$ and

$$
\text { (38.1) } A_{\alpha}^{\prime \prime}=A_{\alpha} \text { for } \alpha \text { cogredient with } \mathbf{A} \text {. }
$$

Now denote by $\alpha \lambda$ the coverings of $\mathbf{K}$. To any $\alpha \lambda=\left\{o_{1}, \cdots, o_{p}, \cdots, o_{s}\right\}$ corresponds the covering

$$
\alpha=\mathbf{A} \alpha \lambda=\left\{\mathbf{A} o_{1}, \cdots, \mathbf{A} o_{p}\right\}
$$

of $\mathbf{A}$ where the $\mathbf{A} o_{i}=\mathbf{A} \cap o_{i}$ are "indexed sets" (in the sense of $[1$, p. 72, §5]), that is $\mathbf{A} o_{i}$ and $\mathbf{A} o_{j}$ are considered as different if $i \neq j$.

Each covering $\alpha$ of $\mathbf{A}$ can be put in the form $\alpha=\mathbf{A} \alpha \lambda$. The nerve of $\mathbf{A} \alpha \lambda$ being $A_{\alpha \lambda}^{\prime \prime}$ there is a natural isomorphism between $\nabla_{\mathbf{A}}^{r}, \Delta_{\mathbf{A}}^{r}$ and

$$
\nabla_{A^{\prime \prime}}^{r}=\lim \sup \left(\nabla_{A_{\alpha \lambda}^{\prime \prime},}^{r}, \pi_{\beta \mu 0}^{\alpha \lambda 0}\right) \quad, \quad \Delta_{A^{\prime \prime}}^{r}=\lim \inf \left(\Delta_{A_{\alpha \lambda}^{\prime \prime},}^{r}, \varpi_{\alpha \lambda 0}^{\beta \mu 0}\right) .
$$

By virtue of the identity (38.1) this isomorphism turns into an isomorphism between $\nabla_{\mathbf{A}}^{r}, \Delta_{\mathbf{A}}^{r}$ and $\nabla_{A}^{r}, \Delta_{A}^{r}$ which is our natural isomorphism $(\mathbf{A} A)$.

\section{\$10. The case of continuous polyhedrons $\mathbf{K}$ and $\mathbf{A} \subseteq \mathbf{K}$}

39. An invariance theorem. Let $\boldsymbol{K}$ be a finite polyhedron, $K$ a simplicial decomposition (Simplizialzerlegung) of $\mathbf{K}$ (terminology of [4, pp. 128-129]). 
Let $A$ be a closed subcomplex of the complex $K$; then $\mathbf{A}$ denotes the polyhedron composed by the simplices of $A$; as usual $G=K \backslash A, \Gamma=\mathbf{K} \backslash \mathbf{A}$. Let us show that the groups of the figure $\mathbf{K}, \mathbf{A}, \boldsymbol{\Gamma}$ are isomorphic to those of the figure $K, A, G$. To this end denote by

$$
K_{1}, K_{2}, \cdots, K_{n}, \cdots
$$

the successive barycentric subdivisions of $K$; then the $n$th barycentric subdivision $A_{n}$ of $A$ is a subcomplex of $K_{n}$. Denote for a moment by $\lambda_{n}^{\prime}$ the covering of $\mathbf{K}$ by the closed barycentric stars of the vertices of $K_{n}$ and take the open neighborhoods of the elements of $\lambda_{n}^{\prime}$ so closely as to get an open covering $\lambda_{n}$ of $\boldsymbol{K}$ similar to $\lambda_{n}^{\prime}$ (that is, having the same nerve as $\lambda_{n}^{\prime}$ ) and cogredient with A. Put $\alpha_{n}=\mathbf{A} \lambda_{n}$. Obviously the $\lambda_{n}$ and the $\alpha_{n}$ form cofinal parts of the sets of all coverings of $\mathbf{K}$ and of $\mathbf{A}$ respectively. The nerve of $\lambda_{n}$ being $K_{n}$, and $A_{n}$ being the nerve of $\alpha_{n}$, the projections of $K_{n+1}$ into $K_{n}$ and of $A_{n+1}$ into $A_{n}$ map any vertex $e_{n+1}$ of $K_{n+1}$ (of $A_{n+1}$ ) on a vertex of the carrier of $e_{n+1}$ in $K_{n}$ (in $A_{n}$ ) and thus are "natural displacements" ("natürliche Verschiebungen" in the sense of $[4$, p. 349]). Therefore by a well known standard process the elements of $\Delta_{\mathbf{K}}^{r}, \Delta_{\mathbf{A}}^{r}$ are in (1-1)-correspondence with the elements of $\Delta_{K}^{r}, \Delta_{A}^{r}$, this correspondence realizing an isomorphism between

$$
\begin{aligned}
& \Delta_{\mathbf{K}}^{r} \quad \text { and } \Delta_{K}^{r}, \quad \Delta_{\mathbf{A}}^{r} \text { and } \Delta_{A}^{r} \text {, } \\
& \Delta_{\mathbf{K A}}^{r} \text { and } \Delta_{K A}^{r}, \quad \Delta_{\mathbf{A}: \mathbf{K}}^{r} \text { and } \Delta_{A: K}^{r} \text {. }
\end{aligned}
$$

All other groups of the figures $\mathbf{K}, \mathbf{A}, \boldsymbol{\Gamma}$ and $K, A, G$ can be derived respectively from $\Delta_{\mathbf{K}}^{r}, \Delta_{\mathbf{K A}}^{r}, \Delta_{\mathbf{A}: \mathbf{K}}^{r}$, and $\Delta_{K}^{r}, \Delta_{K A}^{r}, \Delta_{A: K}^{r}$ by the same algebraic constructions; thus the groups of $\mathbf{K}, \mathbf{A}, \boldsymbol{\Gamma}$ are respectively isomorphic to the groups of $K, A, G$.

40. The general polyhedral case. Let $\mathbf{K}$ and $\mathbf{A} \subseteq \mathbf{K}$ be continuous polyhedrons (that is, topological images of finite polyhedrons). In this section coefficient-groups are the so called "elementary groups," that is discrete groups with a finite number of generators and their (bicompact) character groups. Any subgroup and any difference group of an elementary group being elementary, it follows that the groups $\Delta_{\mathbf{K}}^{r}, \nabla_{\mathbf{K}}^{r}, \Delta_{\mathbf{A}}^{r}, \nabla_{\mathbf{A}}^{r}, \Delta_{\mathbf{K A}}^{r}, \nabla_{\mathbf{K} \mathbf{\Gamma}}^{r}, \Delta_{\mathbf{A}: \mathbf{K}}^{r}, \nabla_{\mathbf{A K}}^{r}, \nabla_{\mathbf{A}: \mathbf{K}}^{r}$, as well as (by the third pair of isomorphisms) the groups $\Delta_{\Gamma: K}^{r}, \nabla_{\Gamma: K^{r}}^{\text {for } r \geqq 1}$ are elementary. Now if - for a discrete group $X$-a subgroup $U$ and the difference group $X-U$ are both elementary, then $X$ itself is elementary (the proof is obvious, see for instance $[4$, p. 576, section 38]). By this lemma and the first pair of isomorphisms the groups $\Delta_{\Gamma}^{r}, \nabla_{\Gamma}^{r}$ for $r \geqq 1$ are elementary. If $X$ is the coefficient-group then, as is easily seen, $\nabla_{\Gamma}^{0}(X)$ is the direct sum of as many groups isomorphic to the group $X$ as there are compact components of $\Gamma$. As in our case the number of these components is finite, $\nabla_{\Gamma}^{0}$ is elementary.

40.1. Thus if $\mathbf{K}$ and $\mathbf{A}$ are continuous polyhedrons, then with respect to 
any elementary coefficient-group all groups of the figure $\mathbf{K}, \mathbf{A}, \boldsymbol{\Gamma}$ are elementary; they are all finite if the coefficient-group is finite.

For a finite coefficient-group $X$ the order of the group $\nabla_{\mathbf{K}}^{r}$ is equal (by the second pair of isomorphisms) to the product of the orders of $\nabla_{\mathbf{K} \boldsymbol{\Gamma}}^{r}$ and $\nabla_{\mathbf{A K}}^{r}$. This holds in particular for $X=I_{m}$ whatever the integer $m \geqq 2$. Since by a theorem of M. Bockstein [6] all groups $\nabla_{\mathbf{K}}^{r}(I)$ are completely determined by the orders of the groups $\nabla_{\mathbf{K}}^{r}(m)$ taken for all $m$ and all $r$, it follows that all $\nabla_{\mathbf{K}}^{r}$ are determined by $\nabla_{\mathbf{K} \boldsymbol{\Gamma}}^{r}(m)$ and $\nabla_{\mathbf{A K}}^{r}(m)$ (taken for all $m$ and all $r$ ). In the same way by the first pair of isomorphisms the groups $\Delta_{\mathbf{A}}^{r}$ are determined by $\Delta_{\mathbf{A}: \mathbf{K}}^{r}(m)$ and $\Delta_{\mathbf{K A}}^{r}(m)$ taken for all $m$ and all $r$. To get an analogous result for the groups $\nabla_{\boldsymbol{\Gamma}}^{r}, \nabla_{\boldsymbol{\Gamma}: \mathbf{K}}^{r}, \nabla_{\mathbf{K} \boldsymbol{\Gamma}}^{r}$ we need the following

LEMma. The groups $\nabla_{\boldsymbol{\Gamma}}^{r}(m)$ (even the groups $\nabla_{\boldsymbol{\Gamma}}^{r}(X)$ for any $\left.X\right)$ can be deduced from the groups $\nabla_{\mathbf{\Gamma}}^{r}(I)$ (taken for all $r$ ) by means of the same relations as in the case where $\Gamma$ is a finite cell complex.

Let us assume for a moment that this lemma is proved. Then as the Bockstein theorem for a complex $K$ rests solely upon the algebraic relations between $\nabla_{K}^{\tau}(I)$ and $\nabla_{K}^{r}(m)$, its original proof still holds for $\Gamma$. Thus our lemma implies the following:

BocKSTEIN THEOREM FOR $\boldsymbol{\Gamma}$. The groups $\nabla_{\boldsymbol{\Gamma}}^{\boldsymbol{r}}(I)$ are completely determined by the orders of the groups $\nabla_{\Gamma}^{r}(m)$ taken for all $m$ and all $r$.

Now by the first pair of isomorphisms the order of $\nabla_{\Gamma}^{r}(m)$ is equal to the product of the orders of $\nabla_{\boldsymbol{\Gamma}: \mathbf{K}}^{r}(m)$ and $\nabla_{\mathbf{K} \boldsymbol{\Gamma}}^{r}(m)$, and therefore $\nabla_{\mathbf{\Gamma}}^{r}(I)$ is determined by the groups $\nabla_{\boldsymbol{\Gamma}: \mathbf{K}}^{r}(m)$ and $\nabla_{\mathbf{K} \boldsymbol{\Gamma}}^{r}(m)$.

It remains only to prove the lemma. A proof of it is contained in the construction of Steenrod [13, section 11] which in our case gives the desired expression. But one can proceed more quickly by the following argument of Bockstein which uses only the final result of Steenrod and not his intermediate construction. The groups $\Delta_{\boldsymbol{\Gamma}}^{r}(I)$ being groups with a finite number of generators, they are respectively isomorphic to the groups $\nabla_{N}^{r}(I)$ where $N$ is an appropriate nerve (see for example $[4$, p. 266, section 9]). Let $\mathbf{N}$ be the corresponding polyhedron. By the fundamental theorem of Steenrod the groups $\nabla_{\mathbf{N}}^{r}(m)$ can be expressed through the groups $\nabla_{\mathbf{N}}^{r}(I)$ and these expressions depend only on the groups involved (and the given coefficient-group, in our case $I_{m}$ ) and are therefore the same for $\boldsymbol{\Gamma}$ and $\mathbf{N}$. On the other hand, the groups $\nabla_{\mathbf{N}}^{r}(I), \nabla_{\mathbf{N}}^{r}(m)$ are isomorphic to $\nabla_{N}^{r}(I), \nabla_{N}^{r}(m)$ and the expression of $\nabla_{\mathbf{N}}^{r}(m)$ through the groups $\nabla_{\mathbf{N}}^{r}(I)$ is the same as the expression of $\nabla_{N}^{r}(m)$ through the groups $\nabla_{N}^{r}(I)$, which proves the lemma.

Thus all results announced in section 14 are now completely proved. Let us remark that the theorems of section 18 could be extended by the same methods first to spectra and then to spaces; such an extension does not present any serious difficulty and may be left to the reader. 


\section{Chapter IV. Manifolds}

\section{\$11. INFINITE COMPLEXES}

41. Infinite complexes $\left({ }^{7}\right)$. Let $Q$ be an infinite locally finite, complete (section 28), simplicial complex, thus a simplicial decomposition of a locally bicompact space (of an infinite polyhedron) $\mathbf{Q}$. Denote by $L_{Q}^{r}$ the group of the $r$-dimensional finite chains on $Q$ with respect to the discrete coefficient-group $X$, by $\bar{L}_{Q}^{r}$ the topologized (as in $[13$, p. 691]) group of all (infinite) $r$-dimensional chains on $Q$ with |respect to the bicompact coefficient-group $\Xi \mid X$. The topology in $\bar{L}_{Q}^{r}$ is that which makes of $\bar{L}_{Q}^{r}$ the direct sum of the groups $\bar{L}_{t}^{r}$, the group $\bar{L}_{i}^{r} \approx \Xi$ being the group of all chains of the form $\bar{a} t_{i}^{r}$, where $t_{i}^{r}$ is an oriented simplex of $Q$ and $\bar{a}$ runs over $\Xi$. Since $L_{Q}^{r}$ is the direct sum of the discrete groups $L_{i}^{r} \approx X$ (the elements of $L_{i}^{r}$ being the chains $a t_{i}^{r}$ with $a$ running over $X$ ), the groups $L_{Q}^{r}$ and $\bar{L}_{Q}^{r}$ are dual to each other. With these groups we get in the usual way the groups $\Delta_{Q}^{r}$ and $\nabla_{Q}^{r}$ : the group $\nabla_{Q}^{r}$ (the group $\Delta_{Q}^{r}$ ) is the difference group of the group of all $r$-dimensional finite $\nabla$-cycles (infinite $\Delta$-cycles) over the subgroup of the bounding ones (a bounding $\nabla$-cycle bounds by definition a finite chain).

The purpose of this section is the proof of the following theorem:

41.1. If $Q$ is a simplicial decomposition of the infinite polyhedron $\mathbf{Q}$, then

$$
\Delta_{\mathbf{Q}}^{r} \approx \Delta_{Q}^{r} ; \quad \nabla_{Q}^{r} \approx \nabla_{Q}^{r} .
$$

By virtue of the dualities $\Delta_{Q}^{r}\left|\nabla_{\mathbf{Q}}^{r}, \Delta_{Q}^{r}\right| \nabla_{Q}^{r}$ it is sufficient to prove the first of the isomorphisms (41.1).

Preliminary Remarks. Let $\alpha$ be a (finite open) covering of $\mathbf{Q}$; an element of $\alpha$ (as well as the corresponding vertex of the nerve $N_{\alpha}$ ) is called regular if its closure is bicompact; the other elements of $\alpha$ (and vertices of $N_{\alpha}$ ) are called special. The simplices of $N_{\alpha}$ whose vertices are all special form the special subcomplex $C_{\alpha}$ of $N_{\alpha}$; the open subcomplex $K_{\alpha}=N_{\alpha} \backslash C_{\alpha}$ is called the regular part of $N_{\alpha}$; all regular vertices of $N_{\alpha}$, and only the regular vertices, are zero-dimensional elements of $K_{\alpha}$.

The open finite subcomplex $G$ of $Q$ is called a regular subcomplex of $Q$ if it is the sum of the open $\operatorname{stars}\left({ }^{8}\right)$ of some of the vertices of $Q$, or (which is the same) if each simplex of $G$ has at least one vertex which belongs to $G$ as an element. The proof of the following statement is obvious: If $G$ is any open finite subcomplex of $Q$, while $Q_{1}, G_{1}$ are barycentric subdivisions of $Q, G$ respectively, then $G_{1}$ is a regular subcomplex of $Q_{1}$.

Our last auxiliary definition in this section is the following: A covering $\alpha$ of $Q$ is called combinatorial with respect to $Q$ if the following conditions are satisfied:

(7) This section will not be needed in the rest of this paper.

( ${ }^{8}$ ) We use the word "star" for complexes as well as for the corresponding point sets. 
(a) Each regular element of $\alpha$ is the open star of some vertex of $Q$.

(b) Let $G_{\alpha}$ be the (regular) subcomplex of $Q$ defined as the sum of all open stars which are elements of $\alpha$; let $\boldsymbol{\Gamma}_{\alpha}$ be the corresponding point set in $\mathbf{Q}$ and $\overline{\mathbf{\Gamma}}$ its closure (in $\mathbf{Q}$ ). Any special element of $\alpha$ either has no points in common with $\overline{\boldsymbol{\Gamma}}$ or is of the form $o_{\alpha t}^{\prime} \cup O_{h}$, where $O_{h}$ is on $\mathbf{Q} \backslash \overline{\boldsymbol{\Gamma}}$ and $o_{\alpha t}^{\prime}$ is the open star (with respect to $Q$ ) of a vertex of some simplex of $G_{\alpha}$.

LEMma 41.2. The regular part of the nerve $N_{\alpha}$ of any covering $\alpha$ combinatorial with respect to $Q$ is a regular subcomplex of $Q$ and any regular subcomplex $G$ of $Q$ is the regular part of the nerve of some covering combinatorial with respect to $Q$.

The first statement of Lemma $\mathbf{4 1 . 2}$ follows immediately from the definition of a covering combinatorial with respect to $Q$. Let us prove the second statement. Let $\boldsymbol{e}_{\boldsymbol{i}}$ be a vertex of an element of $G$. If $\boldsymbol{e}_{i} \in G$ denote by $\boldsymbol{o}_{\boldsymbol{i}}$ the open star of $e_{i}$; if $e_{i}$ is not an element of $G$ put $o_{i}=o_{i}^{\prime} \cup(\mathbf{Q} \backslash \bar{\Gamma})$, where $o_{i}^{\prime}$ is the open star of $e_{i}$ in $Q$. The open sets $o_{i}$ form the required covering.

LEMMA 41.3. Each covering $\alpha$ of $Q$ has a refinement which is combinatorial with respect to a certain subdivision $Q_{\lambda}$ of $Q$.

Proof. Let $o_{1}, \cdots, o_{p}$ be the regular and $o_{p+1}, \cdots, o_{s}$ the special elements of $\alpha$. Take the closed sets $a_{i} \subseteq o_{i}$ in such a way as to get a closed covering $\left\{a_{1}, \cdots, a_{s}\right\}$ of $\mathbf{Q}$. The $a_{i}$ with $i \leqq p$ are bicompact while the $a_{p+1}, \cdots, a_{s}$ may be supposed to be not bicompact. Choose the subdivision $Q_{\lambda}$ of $Q$ such that any star of $Q_{\lambda}$ meeting $a_{i}$ is on $o_{i}$. Now let $o_{\lambda 1}, \cdots, o_{\lambda u}$ be all stars of vertices of $Q_{\lambda}$ which meet any one of the sets $a_{1}, \cdots, a_{p}$. These stars form a regular subcomplex $G_{\lambda}$ of $Q_{\lambda}$; denote by $\Gamma_{\lambda}$ the corresponding subset of $\mathbf{Q}$. Denote by $O_{h}, h=\mathrm{p}+1, \cdots, s$, the open sets composed by all stars of $Q_{\lambda}$ which have points in common with $a_{h}$. Among all zero-dimensional elements of $Q_{\lambda} \backslash G_{\lambda}$ denote by $e_{1}^{\prime}, \cdots, e_{q}^{\prime}$ those which are vertices of elements of $G_{\lambda}$. Define for $i=1,2, \cdots, q$

$$
o_{\lambda, u+i}=o_{\lambda i}^{\prime} \cup\left(O_{h_{i}} \backslash \overline{\boldsymbol{\Gamma}}_{\lambda}\right)
$$

where $o_{\lambda i}^{\prime}$ is the star of $e_{i}^{\prime}$ in $Q_{\lambda}$ and $h_{i} \geqq p+1$ is chosen under the condition that $a_{h_{i}}$ has points in common with $o_{\lambda i}^{\prime}$. By the definition of $O_{h}$ we have

$$
o_{\lambda_{i}}^{\prime} \subseteq O_{h_{i}} \subseteq o_{h_{i}}
$$

thus $o_{\lambda, u+i} \subseteq o_{h_{i}}$. If in the latter construction $O_{p+1}, \cdots, O_{p+q^{\prime}}$ are the only sets $O_{h}$ actually used, we put, for $j=q^{\prime}+1, \cdots, s-p$,

$$
o_{\lambda, u+q+j}=O_{p+j} \backslash \overline{\boldsymbol{\Gamma}}_{\lambda} .
$$

The open sets $o_{\lambda i}, i=1,2, \cdots, u+q+s-p$, form the required covering. 
Now we go over to the proof of the isomorphism $\Delta_{\mathbf{Q}}^{r} \approx \Delta_{Q}^{r}$. Retain only those coverings of $\mathbf{Q}$ which are combinatorial with respect to subdivisions of $Q$. If $\alpha, \beta$ are combinatorial with respect to $Q_{\lambda}, Q_{\mu}$ then we $\operatorname{set} \beta>\alpha$ if both of the following conditions are satisfied:

1. $\beta$ is a refinement of $\alpha, \alpha$ is not a refinement of $\beta$.

2. $Q_{\mu}$ is a subdivision of $Q_{\lambda}$.

The coverings with this ordering form a cofinal part of the set of all coverings of $Q$. The subcomplexes $K_{\alpha}=N_{\alpha} \backslash C_{\alpha}$ being open (even regular) subcomplexes of $Q_{\lambda}$, they undergo projections generated by natural displacements (section 39) of $Q_{\mu}$ into $Q_{\lambda}$. Let $\varpi_{\alpha}^{\beta}$ be those projections. Then

$$
\Delta_{\mathbf{Q}}^{r}=\lim \inf \left(\Delta_{K_{\alpha}}^{r}, \varpi_{\alpha}^{\beta}\right) \text {. }
$$

Take on the other hand all finite open subcomplexes $G_{\alpha \lambda}$ of all subdivisions $Q_{\lambda}$ of $Q$. We say that $G_{\beta \mu}$ follows on $G_{\alpha \lambda}$ and write $\beta \mu>\alpha \lambda$ if $Q_{\mu}$ is a subdivision of $Q_{\lambda}$ and the subdivision $s_{\mu}^{\lambda} G_{\alpha \lambda}$ of $G_{\alpha \lambda}$ in $Q_{\mu}$ is a subcomplex of $G_{\beta \mu}$. In particular, if $G_{\alpha}$ are the subcomplexes of $Q$ itself, then $\beta>\alpha$ means that $G_{\alpha} \subset G_{\beta}$. Denote by $\sigma_{\lambda}^{\mu}$ the natural displacements of $Q_{\mu}$ into $Q_{\lambda}$ and write (as indices) $(\beta \mu) \lambda$ instead of $\sigma_{\lambda}^{\mu} G_{\beta \mu}$ and $\alpha \lambda$ instead of $G_{\alpha \lambda}$. Since $G_{\alpha \lambda}$ is an open subcomplex of $\sigma_{\lambda}^{\mu} G_{\beta \mu}$ we may define a projection $\rho_{\alpha \lambda}^{\beta \mu}$ of the cell complex $G_{\beta \mu}$ into $G_{\alpha \lambda}$ by means of

$$
\rho_{\alpha \lambda}^{\beta \mu} x_{\beta \mu}=J_{\alpha \lambda}^{(\beta \mu) \lambda} \sigma_{\lambda}^{\mu} x_{\beta \mu}
$$

for any chain $x_{\beta \mu}$ on $G_{\beta \mu}$. This gives for $\mu=\lambda$ the relation $\rho_{\alpha \lambda}^{\beta \lambda} x_{\beta \lambda}=J_{\alpha \lambda}^{\beta \lambda} x_{\beta \lambda}$ and in particular $\rho_{\alpha}^{\beta} x_{\beta}=J_{\alpha}^{\beta} x_{\beta}$ on $Q$.

Those $G_{\alpha \lambda}$ which are subdivisions of appropriate $G_{\alpha} \subset Q$ form a cofinal part of the set of all $G_{\alpha \lambda}$, and $\rho_{\alpha}^{\alpha \lambda}$ maps isomorphically $\Delta_{\alpha \lambda}^{r}$ on $\Delta_{\alpha}^{r}$. Therefore $\lim \inf \left(\Delta_{\alpha \lambda}^{r}, \rho_{\alpha \lambda}^{\beta \mu}\right) \approx \lim \inf \left(\Delta_{\alpha}^{r}, J_{\alpha}^{\beta}\right)$. But the groups $\lim \inf \left(\Delta_{\alpha}^{r}, J_{\alpha}^{\beta}\right)$ and $\Delta_{Q}^{r}$ are isomorphic as shown by Steenrod [13, pp. 691-692]; thus

$$
\Delta_{Q}^{r} \approx \lim \inf \left(\Delta_{\alpha \lambda}^{r}, \rho_{\alpha \lambda}^{\beta \mu}\right) \text {. }
$$

On the other hand, since the regular subcomplexes form a cofinal part in the set of all $G_{\alpha \lambda}$, we deduce from Lemma 41.2 the isomorphism

$$
\Delta_{\mathbf{Q}}^{r} \approx \lim \inf \left(\Delta_{\alpha \lambda}^{r}, \rho_{\alpha \lambda}^{\beta \mu}\right) \text {. }
$$

From (41.5) and (41.6) follows $\Delta_{Q}^{r} \approx \Delta_{\mathbf{Q}}^{r}$, q.e.d.

42. The groups $\delta_{Q}^{r}$ and the duality theorem of Alexander-Pontrjagin. Let $X$ be a discrete coefficient-group. Denote by $\delta_{Q}^{r}$ the difference group of the group of all $r$-dimensional finite $\Delta$-cycles of the (infinite) complex $Q$ over the subgroup of the bounding cycles (a bounding cycle bounds by definition a finite chain). In this section we suppose that $Q$ is an $n$-dimensional combinatorial ( $h$-)manifold (finite or infinite), that is, that for the open star $O_{i}$ of any vertex $e_{i}$ of $Q$ 


$$
\Delta_{o_{i}}^{n}(I) \approx \nabla_{o_{i}}^{n}(I) \approx I,
$$

while for $r<n$ the groups $\Delta_{O_{i}}^{r}(I) \approx \nabla_{O_{i}}^{r}(I)$ contain the zero element only. We suppose moreover that $Q$ is orientable, which means that for every component $Q_{i}$ of $Q, \Delta_{Q_{i}}^{n}(I) \approx \nabla_{Q_{i}}^{n}(I) \approx I$. The oriented (open) barycentric stars of $Q$ form a cell complex $Q^{*}$. If $t_{i}^{p}$ is an oriented simplex of $Q$, then we denote by $\tau_{i}^{q}$, with $q=n-p$, the barycentric star with the center in the center of $t_{i}^{p}$ oriented in such a way that the intersection number $\left(t_{i}^{p} \times \tau_{i}^{q}\right)$ be equal to +1 (see for example $[12, \S 69]$ ). Then we have in the cell complex $Q^{*}$ the incidence numbers

$$
\left(\tau_{j}^{q+1}: \tau_{i}^{q}\right)=(-1)^{p}\left(t_{i}^{p}: t_{j}^{p-1}\right)
$$

Define now for any $p$-chain $x^{p}$ on $Q$ the $q$-chain $D^{q} x^{p}$ on $Q^{*}$ taking on $\tau_{i}^{q}$ the same value as $x^{p}$ takes on $t_{i}^{p}$. An easy calculation shows that

$$
\Delta D^{q} x^{p}=(-1)^{p+1} D^{q-1} \nabla x^{p}
$$

whence it follows that the operator $D^{q}$ generates an isomorphism between $\nabla_{Q}^{p}$ and $\delta_{Q^{*}}^{q}$. As both $\delta_{Q}^{q}$ and $\delta_{Q^{*}}^{q}$ are isomorphic to $\delta_{Q_{1}}^{q}$, where $Q_{1}$ is the barycentric subdivision of $Q$, we have the

Duality theorem of Poincaré.

$$
\nabla_{Q}^{p} \approx \delta_{Q}^{q}, \quad q=n-p .
$$

Let $\mathbf{Q}$ be an $n$-dimensional continuous manifold, that is, a finite or infinite polyhedron, one and thus all simplicial decompositions of which are combinatorial manifolds. Then any open set on $\mathbf{Q}$ is also a continuous manifold (see [4, pp. 143-146, Theorem "of Runge"]). There follows then from the duality theorems of Kolmogoroff and of Poincaré the

Duality theorem of Aiexander-Pontruagin. If the $n$-dimensional orientable continuous manifold $\mathbf{Q}$ is simply connected in the dimensions $p$ and $p+1$, $\mathbf{A}$ is a closed set on $\mathbf{Q}$, and $\mathbf{\Gamma}=\mathbf{Q} \backslash \mathbf{A}$, then the groups $\Delta_{\mathbf{A}}^{p}$ and $\delta_{\mathbf{\Gamma}}^{q-1}$ are dual (the groups $\nabla_{\mathbf{A}}^{p}$ and $\delta_{\mathbf{\Gamma}}^{q-1}$ are isomorphic) to each other. Here the coefficient-group for $\delta_{\mathbf{\Gamma}}^{q-1}$ and $\nabla_{\mathbf{A}}^{p}$ is the discrete group $X$ while the coefficient-group for $\Delta_{\mathbf{A}}^{p}$ is the bicompact group $\Xi \mid X$.

The generalization of this theorem for an arbitrary orientable manifold (not necessarily simply connected in any dimension) is given by the

General Alexander-Pontruagin DUality theorem. For any $n$-dimensional orientable continuous manifold $\mathbf{K}$, any closed set $\mathbf{A} \subseteq \mathbf{K}$, and $\mathbf{\Gamma}=\mathbf{K} \backslash \mathbf{A}$, the group $\nabla_{\mathbf{A}: \mathbf{K}}^{p}$ is isomorphic to the group $\delta_{\mathbf{\Gamma}: \mathbf{K}}^{q-1}$, where the latter group is defined as the difference group of the r-dimensional finite cycles on $\boldsymbol{\Gamma}$ over the subgroup of the cycles bounding on $\boldsymbol{\Gamma}$ (coefficient-group discrete). 
Here the finite cycles on $\boldsymbol{\Gamma}$ are to be understood in some invariant sense (as "continuous cycles" in the sense of [4, pp. 333-339] or as convergent cycles on compact sets $\phi \subset \Gamma$ (section 43$)$ ) or to be replaced by the $\delta$-classes of section 43.

To prove this theorem we have to show only that the groups $\nabla_{\Gamma: \kappa}^{r}$ and $\delta_{\boldsymbol{\Gamma}}^{r}: \mathbf{K}$ are isomorphic. This can be done by the standard methods of proving invariance theorems (see for example [4, Chapter IX] and [3]); these methods, however, so far as the writer sees, need at a certain moment the Combinatorial Lemma 45.3. But the same lemma is the only essential point in proving directly the isomorphism $\nabla_{\mathbf{A}: \mathbf{K}}^{p} \approx \delta_{\mathbf{\Gamma}: \mathbf{K}}^{q-1}$. We prefer therefore to give this direct proof which rests entirely on section 15 (Chapter I), being practically independent of Chapters II and III.

\section{§12. The gRoups $\Delta_{\mathbf{A}: \mathbf{K}}^{r}, \nabla_{\mathbf{A}: \mathbf{K}}^{r}$, AND $\delta_{\boldsymbol{\Gamma}: \mathbf{K}}^{r}$ FOR A MANifold $\mathbf{K}$}

43. The groups $\Delta_{\mathbf{A}: \mathbf{K}}^{r}$ and $\nabla_{\mathbf{A}: \mathbf{K}}^{r}$ for a polyhedron $\mathbf{K}$. Let $\mathbf{K}$ be a polyhedron; we suppose this polyhedron finite (that is bicompact) although the follcwing definitions hold for infinite polyhedrons too. As usual $\mathbf{A}$ is any closed set in $\mathbf{K}$ and $\Gamma=K \backslash A$. In this case the groups $\Delta_{\mathbf{A}: \mathbf{K}}^{r}, \nabla_{\mathbf{A}: \mathbf{K}}^{r}$ can be defined in a quite elementary way, independent of the definitions and results of Chapters II and III. Let

$$
K_{1}, K_{2}, \cdots, K_{\alpha}, \cdots
$$

be a sequence of successive subdivisions of a given simplicial subdivision $K$ of $\mathbf{K}$; we suppose moreover that for any integer $\alpha \geqq 1$ the complex $K_{\alpha+1}$ is the result of one or more successive barycentric subdivisions of $K_{\alpha}$. Taking in $K_{\alpha}$ all simplices which meet $\mathbf{A}$ and all faces of these simplices we get a closed subcomplex $A_{\alpha}$ of $K_{\alpha}$. Put in correspondence to any vertex $e_{\alpha+1}$ of $K_{\alpha+1}$ a fixed vertex $e_{\alpha}=\sigma_{\alpha}^{\alpha+1} e_{\alpha+1}$ of the carrier of $e_{\alpha+1}$ in $K_{\alpha}$. This gives a well defined simplicial mapping (a "natural displacement," section 39) called a projection of $K_{\alpha+1}$ on to $K_{\alpha}$. The projection $\sigma_{\alpha}^{\alpha+1} \operatorname{maps} A_{\alpha+1}$ in to $A_{\alpha}$. For $\beta>\alpha+1$ put

$$
\stackrel{\beta}{\sigma_{\alpha}}=\sigma_{\alpha}^{\alpha+1} \sigma_{\alpha+1}^{\alpha+2} \cdots \sigma_{\beta-1}^{\beta}
$$

which implies the transitivity relation $\sigma_{\alpha}^{\gamma}=\sigma_{\alpha}^{\beta} \sigma_{\beta}^{\gamma}$ for any $\gamma>\beta>\alpha$.

The projection $\sigma_{\alpha}^{\beta}$ generates a homomorphism $\omega_{\alpha}^{\beta}$ of $L_{\beta}^{r}=L_{K_{\beta}}^{r}$ in to $L_{\alpha}^{r}=L_{K_{\alpha}}^{r}$ and a homomorphism $\varpi_{\alpha 0}^{\beta 0}$ of $L_{\beta 0}^{r}=L_{A}^{r}$ into $L_{\alpha 0}^{r}=L_{A_{\alpha}}^{r}$; the conjugate homomorphisms $\pi_{\beta}^{\alpha}$ and $\pi_{\beta 0}^{\alpha 0}$ of the group $L_{\alpha}^{r}$ into $L_{\beta}^{r}$ and of $L_{\alpha 0}^{r}$ into $L_{\beta 0}^{r}$ are defined by

$$
\left(\stackrel{\alpha}{\pi_{\beta}} x_{\alpha}^{r} \cdot t_{\beta}^{r}\right)=\left(\stackrel{r}{x_{\alpha} \cdot \sigma_{\alpha}^{\beta} t_{\beta}^{r}}\right)
$$

for any chain $x_{\alpha}^{r}$ on $K_{\alpha}$ (on $A_{a}$ ) and any oriented simplex $t_{\beta}^{r}$ of $K_{\beta}$ (of $A_{\beta}$ ). The homomorphisms $\varpi_{\alpha 0}^{\beta 0}, \pi_{\beta 0}^{\alpha 0}$ generate homomorphisms (denoted also by $\varpi_{\alpha 0}^{\beta 0}, \pi_{\beta 0}^{\alpha 0}$ ) of $\Delta_{\beta 0}^{r}$ into $\Delta_{\alpha 0}^{r}$ (of $\nabla_{\alpha 0}^{r}$ into $\nabla_{\beta 0}^{r}$ ) and map $\Delta_{\beta 0 ; \beta}^{r}=\Delta_{A_{\beta}: K_{\beta}}^{r}$ into $\Delta_{\alpha 0: \alpha}^{r}=\Delta_{A_{\alpha}: K_{\alpha}}^{r} ; \nabla_{\alpha 0: \alpha}^{r}=\nabla_{A_{\alpha}: K_{\alpha}}^{r}$ into $\nabla_{\beta 0: \beta}^{r}=\nabla_{A_{\beta}: K_{\beta}}^{r}$. We define now: 


$$
\Delta_{\mathbf{A}}^{r}: \mathbf{K}=\lim \inf \left(\Delta_{\alpha 0: \alpha}^{r}, \varpi_{\alpha 0}^{\beta 0}\right) ; \quad \nabla_{\mathbf{A}}^{r}: \mathbf{K}=\lim \sup \left(\nabla_{\alpha 0: \alpha}^{r}, \underset{\pi_{\beta 0}}{\alpha 0}\right) .
$$

Remark 1. It is easily seen that the groups $\Delta_{\mathbf{A}: \mathbf{K}}^{r}, \nabla_{\mathbf{A}: \mathbf{K}}^{r}$ just defined do not depend upon the special choice of the sequence (43.1). But one can also show that the definitions of this section agree with those of Chapter III. In fact, the simplices of $A_{\alpha}$ are characterized among the simplices of $K_{\alpha}$ by the property that their open stars meet $\mathbf{A}$; therefore $A_{a}$ is the nerve of the covering

$$
\alpha=\left\{o_{\alpha 1}, \cdots, o_{\alpha s}\right\}
$$

of $\mathbf{A}$, where $o_{\alpha i}=\mathbf{A} \cap O_{\alpha i}$ and the $O_{\alpha i}$ are those open stars of (the vertices of) $K_{\alpha}$ which meet $\mathbf{A}$. In other words, if $\alpha \lambda$ is the covering of $\mathbf{K}$ by the open stars of the vertices of $K_{\alpha}$ then in the sense of section 38 we have $\alpha=\mathbf{A} \alpha \lambda$. The coverings $\alpha \lambda, \alpha=1,2, \cdots$, form a confinal part of the set of all coverings of $\mathbf{K}$ and the projections $\sigma_{\alpha}^{\beta}$ put in correspondence to each $O_{\beta j}$ an $O_{\alpha i} \supseteq O_{\beta j}$. If we take into account section 38 we see at once that the definition (43.2) gives us the same groups as the definitions of Chapter III.

REMARK 2. The elements of $\Delta_{\mathbf{A}: \mathbf{K}}^{r}$ are those "threads" or sequences

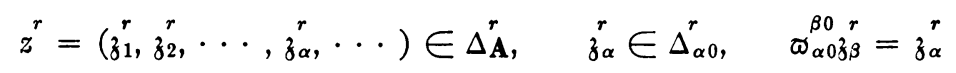

whose elements ${ }_{z_{\alpha}^{r}}^{r} \in \Delta_{\alpha 0}^{r}$ are classes of cycles on $A_{\alpha}$ bounding on $K_{\alpha}$.

It is easy to see that the group $\Delta_{\mathbf{A}: \mathbf{K}}^{r}$ can also be defined as the difference group of the group of all $r$-dimensional convergent cycles (see for instance $[5$, p. 241]) of the set $\mathbf{A}$ over the subgroup of cycles bounding on $\mathbf{K}$.

The elements of $\nabla_{\mathbf{A}: \mathbf{K}}^{r}$ are extension classes of $r$-dimensional $\nabla$-cycles; the $\nabla$-cycle $z_{\alpha}^{r}$ on $A_{\alpha}$ and the $\nabla$-cycle $z_{\beta}^{r}$ on $A_{\beta}$ belong by definition to the same extension class if there exists a $\gamma>\alpha, \beta$ such that $\pi_{\gamma}^{\alpha} z_{\alpha}^{r}-\pi_{\gamma}^{\beta} z_{\beta}^{r}$ is extensible over $K_{\gamma}$.

REMARK 3. The coefficient-groups in $\Delta_{\mathbf{A}: \mathbf{K}}^{r}, \nabla_{\mathbf{A}: \mathbf{K}}^{r}$ are dual: bicompact for $\Delta_{\mathbf{A}: \mathbf{K}}^{r}$ and discrete for $\nabla_{\mathbf{A}: \mathbf{K}}^{r}$.

44. The groups $\delta_{\Gamma: K}^{r}$ for a manifold $\mathbf{K}$. From now to the end of this paper $\boldsymbol{K}$ is a continuous $n$-dimensional manifold (definition in section 42 ) which for sake of simplicity we suppose closed (that is bicompact) although all following definitions and results hold without this restriction. We conserve the notations of section 43 and denote by $K_{\alpha 1}$ the barycentric subdivision of $K_{\alpha}$ (thus $K_{\alpha+1}$ either coincides with $K_{\alpha 1}$ or is a subdivision of $K_{\alpha 1}$ ). The complex of all (open) barycentric stars of $K_{\alpha}$ is denoted by $K_{\alpha}^{*}$. The barycentric stars which correspond to simplices of $G_{\alpha}=K_{\alpha} \backslash A_{\alpha}$ form a closed subcomplex $G_{\alpha}{ }^{*}$ of $K_{\alpha}^{*}$. Since the elements of $K_{\alpha}^{*}$ are subcomplexes of $K_{\alpha 1}$, we get a closed subcomplex $G_{\alpha 1}^{*}$ of $K_{\alpha 1}$, called the barycentric subdivision of $G_{\alpha}^{*}$, if we take all simplices of $K_{\alpha 1}$ lying on elements $T_{\alpha}^{*}$ of $G_{\alpha}^{*}$ (that is all simplices which are elements of the subcomplexes $\left.T_{\alpha}^{*} \in G_{\alpha}{ }^{*}\right)$. The polyhedron composed of all simplices of $G_{\alpha 1}^{*}$ is denoted by $\Gamma_{\alpha 1}^{*}$. It is $\Gamma_{\alpha 1}^{*} \subseteq \Gamma$ and even $\Gamma=\cup_{\alpha=1}^{\infty} \Gamma_{\alpha 1}^{*}$. Without loss of generality we may suppose that $\Gamma_{\alpha 1} \subseteq \Gamma_{(\alpha+1) 1}$. Therefore the subdivision 
of $G_{\alpha 1}^{*}$ into simplices of $K_{\beta 1}, \beta \geqq \alpha$, is a subcomplex $s_{\beta 1}^{\alpha 1} G_{\alpha 1}^{*}$ of $G_{\beta 1}^{*}$, and for any chain $x_{\alpha 1}^{r}$ on $G_{\alpha 1}$ we have the subdivision $s_{\beta 1}^{\alpha 1} x_{\alpha 1}^{r}$ on $G_{\beta 1}^{*}$.

REMARK 1. By definition

$$
\left(s_{\beta 1}^{\alpha 1} x_{\alpha 1}^{r} \cdot t_{\beta 1}^{r}\right)=\left(x_{\alpha 1}^{r} \cdot t_{\alpha 1}^{r}\right)
$$

if the simplex $t_{\beta 1}^{r}$ of $G_{\beta 1}^{*}$ lies on $t_{\alpha 1}^{r}$ and its orientation is coherent with the orientation of $t_{\alpha 1}^{r} ;\left(s_{\beta 1}^{\alpha 1} x_{\alpha 1}^{r} \cdot t_{\beta 1}^{r}\right)=0$ if $t_{\beta 1}^{r}$ lies on a higher dimensional element of $G_{\alpha 1}^{*}$.

REMARK 2. In an analogous way we define the subdivision $s_{\beta 1}^{\alpha} x_{\alpha}^{r}$ of a chain $x_{\alpha}^{r}$ on $G_{\alpha}^{*}$. Obviously for $\beta>\alpha$

$$
\stackrel{\alpha}{s_{\beta 1} x_{\alpha}^{r}}=\stackrel{\alpha 1}{\alpha s_{\beta 1}^{\alpha} s_{\alpha 1}^{r} x_{\alpha}^{r}} .
$$

After these preliminary remarks we define the group $\delta_{\Gamma: \mathbf{K}}^{r}$ as follows. We say that two $\Delta$-cycles, the cycle $z_{\alpha 1}^{\tau}$ on $G_{\alpha 1}^{*}$ and the cycle $z_{\beta 1}^{r}$ on $G_{\beta 1}^{*}$, belong to the same $r$-dimensional $\delta$-class on $\Gamma$ if there is a $\gamma>\alpha, \beta$ such that $s_{\gamma 1}^{\alpha 1} z_{\alpha 1}-s_{\gamma 1}^{\beta 1} z_{\beta 1}^{\gamma}$ bounds on $G_{\gamma 1}^{*}$. To get the sum of two $\delta$-classes $z^{r}$ and $z^{\prime r}$ take ad libitum $z_{\alpha 1}^{r} \in z^{r}, z_{\beta 1}^{r} \in z^{\prime r}$ and define $z^{r}+z^{\prime r}$ as the $\delta$-class containing $s_{\gamma 1}^{\alpha 1} z_{\alpha 1}^{r}+s_{\gamma 1}^{\beta 1} z_{\beta 1}^{r}$ where $\gamma$ is any integer which is greater than or equal to the greatest among the integers $\alpha$ and $\beta$.

Definition 44.1. The discrete additive group of all $r$-dimensional $\delta$-classes on $\Gamma$ is called the group $\delta_{\Gamma}^{r}$ (with respect to the given discrete coefficient-group).

Definition 44.2. The subgroup $\delta_{\Gamma: K}^{r}$ of $\delta_{\Gamma}^{r}$ is formed by the $\delta$-classes $z^{r} \in \delta_{\Gamma}^{r}$ whose elements $z_{\alpha 1}^{r}$ bound on (the corresponding) $K_{\alpha 1 \text {. }}$

Remark 3. The groups $\delta_{\Gamma}^{r}, \delta_{\Gamma: K}^{r}$ could be defined also as follows. A convergent cycle on a compact subset of $\boldsymbol{\Gamma}$ (with respect to a discrete coefficientgroup $X$ ) is called a cycle on $\Gamma$; by definition, it bounds on $\Gamma$ if it bounds on a compact subset of $\boldsymbol{\Gamma}$. The difference group of the group of all $r$-dimensional cycles on $\boldsymbol{\Gamma}$ over the subgroup of the cycles bounding on $\boldsymbol{\Gamma}$ is the group $\delta_{\Gamma}^{r}$; the subgroup of $\delta_{\boldsymbol{\Gamma}}^{r}$ whose elements are classes of cycles bounding on $\mathbf{K}$ is the group $\delta_{\Gamma: K}^{r}$; the latter group can also be defined as the difference group of the group of all $r$-dimensional cycles on $\boldsymbol{\Gamma}$ over the subgroup of the cycles bounding on $\mathbf{K}$.

\section{\$13. The general Alexander-Pontrjagin duality theorem:} REDUCTION TO THE COMBINATORIAL LEMMA

45. Formulation of the theorem and of the lemma. From now on we either suppose the manifold $\mathbf{K}$ orientable or take for the coefficient-group the group $I_{2}$. All notations of $\$ 12$ hold.

The theorem to be proved is:

45.1. The groups $\Delta_{\mathbf{A}: \mathbf{K}}^{p}$ and $\delta_{\Gamma: \kappa}^{q-1}(q=n-p)$ are dual; 
or (equivalent formulation):

45.2. The groups $\nabla_{\mathbf{A}: \mathbf{K}}^{p}$ and $\delta_{\mathbf{\Gamma}: \mathbf{K}}^{q-1}(q=n-p)$ are isomorphic.

The proof rests upon the following

Combinatorial Lemma 45.3. If $z_{\alpha}^{p}$ is a $\nabla$-cycle on $K_{\alpha}$ equal to zero on $A_{\alpha}$ then for any $\beta>\alpha$ we have

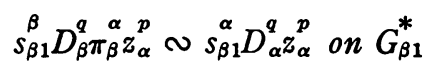

( $\sim$ is the sign of homology, section $9, D^{a}$ is the duality operator of section 42 ).

This lemma will be proved in the next paragraph; in the present paragraph we suppose the lemma proved and prove on this basis the Theorem 45.2. We write $E_{\alpha}$ insteat of $E_{K_{\alpha}}^{A_{\alpha}}$.

46. The operators $\Delta D_{\alpha}^{q} E_{\alpha}$ and the isomorphism $\Delta D^{q} E$. To each $\nabla$-cycle $z_{\alpha 0}^{p}$ on $A_{\alpha}$ corresponds the $\nabla$-cycle $\nabla E_{\alpha} z_{\alpha 0}^{p}$ on $G_{\alpha}$ and thus the $\Delta$-cycle $D_{\alpha}^{q-1} \nabla E_{\alpha} z_{\alpha 0}^{p}=(-1)^{p+1} \Delta D_{\alpha}^{q} E_{\alpha} z_{\alpha 0}^{p}$ on $G_{\alpha}{ }^{*}$ bounding on $K_{\alpha}{ }^{*}$. If a $\nabla$-cycle $z_{\alpha}^{p}$ on $G_{\alpha}$ bounds on $G_{\alpha}$ the chain $x_{\alpha}^{p-1}$, then $D_{\alpha}^{q} z_{\alpha}^{p}$ bounds on $G_{\alpha}^{*}$ the chain $\pm D_{\alpha}^{q+1} x_{\alpha}^{p-1}$ and vice versa. Therefore from section 15 we have:

46.1. The operator $\Delta D_{\alpha}^{q} E_{\alpha}$ produces an isomorphism of the group $\nabla_{A_{\alpha}: K_{\alpha}}^{p}$ onto the group $\Delta_{G_{\alpha}: K_{\alpha}}^{q-1}$.

Our purpose is to show that the operators $\Delta D_{\alpha}^{\alpha} E_{\alpha}$ taken for different $\alpha$ produce an isomorphism-we call it $\Delta D^{a} E$ - of $\nabla_{A: K}^{p}$ onto $\delta_{\Gamma: K}^{q-1}$. We need for this proof some minor lemmas.

Lemma 46.2. For any $\nabla$-cycle $z_{\alpha 0}^{p}$ on $A_{\alpha}$ and $\beta>\alpha$

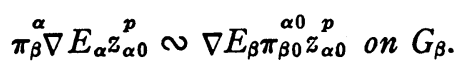

Proof. As $\pi_{\beta}^{\alpha}$ commutes with $\nabla$ we have to prove the homology

$$
\nabla \stackrel{\alpha}{\alpha} E_{\alpha}^{p} z_{\alpha 0}^{p} \sim \nabla E_{\beta} \pi_{\beta 0}^{\alpha 0} z_{\alpha 0}^{p} \text { on } G_{\beta}
$$

or

$$
\nabla\left(\pi_{\beta}^{\alpha} E_{\alpha} z_{\alpha 0}^{p}-E_{\beta} \pi_{\beta 0}^{\alpha 0} z_{\alpha 0}^{p}\right) \sim 0 \text { on } G_{\beta} .
$$

But an easy direct calculation shows that the chain $\pi_{\beta}^{\alpha} E_{\alpha} z_{\alpha 0}^{p}-E_{\beta} \pi_{\beta 0}^{\alpha 0} z_{\alpha 0}^{p}$ is itself on $G_{\beta}$, which proves the assertion.

The fundamental lemma follows:

Lemma 46.3. For any $\nabla$-cycle $z_{\alpha 0}^{p}$ on $A_{\alpha}$ and $\beta>\alpha$

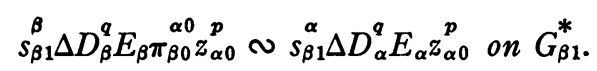

Proof. Set in (45.3)

$$
z_{\alpha}^{p+1}=\nabla E_{\alpha} z_{\alpha 0}^{p}
$$


instead of $z_{\alpha}^{p}$; it becomes

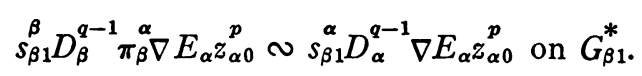

Apply to both sides of (46.2) the operator $D_{\beta}^{q-1}$; this gives

$$
D_{\beta}^{q-1} \underset{\beta}{\alpha} \nabla E_{\alpha} z_{\alpha 0}^{p} \sim D_{\beta}^{q-1} \nabla E_{\beta} \pi_{\beta 0}^{\alpha 0} z_{\alpha 0}^{p} \text { on } G_{\beta}^{*} \text {. }
$$

Thus on the left-hand side of (46.31) we can replace $D_{\beta}^{\alpha-1} \pi_{\beta}^{\alpha} \nabla E_{\alpha} z_{\alpha 0}^{p}$ by $D_{\beta}^{\alpha-1} \nabla E_{\beta} \pi_{\beta 0}^{\alpha 0} z_{\alpha 0}^{p}$ and get

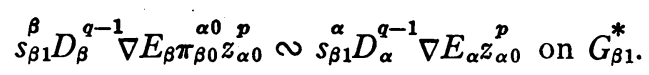

But by (42.1)

$$
\left\{\begin{array}{c}
D_{\beta}^{q-1} \nabla E_{\beta} \pi_{\beta 0}^{\alpha 0} z_{\alpha 0}^{p}=(-1)^{p+1} \Delta D_{\beta}^{q} E_{\beta} \pi_{\beta 0}^{\alpha 0} z_{\alpha 0}^{p}, \\
D_{\alpha}^{q-1} \nabla E_{\alpha} z_{\alpha 0}^{p}=(-1)^{p+1} \Delta D_{\alpha}^{q} E_{\alpha} z_{\alpha 0}^{p} .
\end{array}\right.
$$

If we bring this into (46.32) we get (46.3).

It is now easy to prove that the homomorphisms $\Delta D_{\alpha}^{q} E_{\alpha}$ taken for different $\alpha$ generate a homomorphism $\Delta D^{q} E$ of $\nabla_{\mathbf{A}: \mathbf{K}}^{p}$ into $\delta_{\Gamma: K}^{q-1}:$ since every $\Delta D_{\alpha}^{q} E_{\alpha} z_{\alpha 0}^{p}$ bounds on $K_{\alpha}^{*}$ we need only to verify the following

LEMMA 46.4. If for the $\nabla$-cycles $z_{\alpha 0}^{p}$ on $A_{\alpha}$ and $z_{\beta 0}^{p}$ on $A_{\beta}$ there exists $a \gamma>\alpha, \beta$ such that $\pi_{\gamma 0}^{\alpha 0} z_{\alpha 0}^{p}-\pi_{\gamma 0}^{\beta 0} z_{\beta 0}^{p}$ is extensible over $K_{\gamma}$, then

$$
s_{\gamma 1}^{\alpha} \Delta D_{\alpha}^{q} E_{\alpha} z_{\alpha 0}^{p} \sim s_{\gamma 1}^{\beta} \Delta D_{\beta}^{q} E_{\beta} z_{\beta 0}^{p} \text { on } G_{\gamma 1}^{*} \text {. }
$$
thus

Proof. From 46.1 it follows that $\Delta D_{\gamma}^{q} E_{\gamma}\left(\pi_{\gamma 0}^{\alpha 0} z_{\alpha 0}^{p}-\pi_{\gamma 0}^{\beta 0} z_{\beta 0}^{p}\right)$ bounds on $G_{\gamma}{ }^{*}$,

$$
s_{\gamma 1}^{\gamma} \Delta D_{\gamma}^{q} E_{\gamma} \pi_{\gamma 0 z_{\alpha 0}}^{\alpha 0} \sim s_{\gamma 1}^{\gamma} \Delta D_{\gamma}^{q} E_{\gamma} \pi_{\gamma 0}^{\beta 0} z_{\beta 0}^{p} \text { on } G_{\gamma 1}^{*} \text {. }
$$

On the other hand (46.3) gives (with $\beta$ replaced by $\gamma$ )

$$
s_{\gamma 1}^{\gamma} \Delta D_{\gamma}^{q} E_{\gamma} \pi_{\gamma 0 z_{\alpha 0}}^{\alpha \theta} \sim s_{\gamma 1}^{\alpha} \Delta D_{\alpha}^{q} E_{\alpha} z_{\alpha 0}^{p} \text { on } G_{\gamma 1}^{*}
$$

and (with $\alpha, \beta$ replaced by $\beta, \gamma$ respectively)

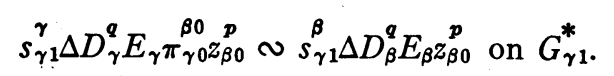

These homologies substituted into (46.41) give (46.4).

46.5. The homomorphism $\Delta D^{q} E$ of $\nabla_{\mathbf{A}: \mathbf{K}}^{p}$ into $\delta_{\Gamma: K}^{q-1}$ is an isomorphism.

This follows from

LEMmA 46.51. If we have for $a \nabla$-cycle $z_{\alpha 0}^{p}$ on $A_{\alpha}$ and a certain $\beta>\alpha$ the homology

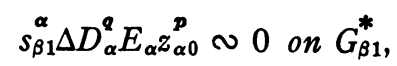

then $\pi_{\beta 0}^{\alpha 0} z_{\alpha 0}^{p}$ is extensible over $K_{\beta}$. 
Proof. By (46.3)

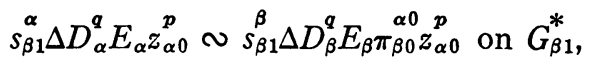

thus $s_{\beta 1}^{\beta} \Delta D_{\beta}^{q} E_{\beta} \pi_{\beta 0}^{\alpha 0} z_{\alpha 0}^{p}$ bounds on $G_{\beta 1}^{*}$. It is known (see for instance $[4$, p. 247 , Theorem III]): If a cycle of the form ${S_{\beta 1}^{\beta}}_{z_{\beta}}^{*}$, where $z_{\beta}^{*}$ is a $\Delta$-cycle on $G_{\beta}^{*}$, bounds on $G_{\beta 1}^{*}$, then $z_{\beta}{ }^{*}$ bounds on $G_{\beta}{ }^{*}$. Therefore $\Delta D_{\beta}^{q} E_{\beta} \pi_{\beta 0}^{\alpha 0} z_{\alpha 0}^{p}$ bounds on $G_{\beta}{ }^{*}$ and by 46.1 the $\nabla$-cycle $\pi_{\beta 0}^{\alpha 0} z_{\alpha 0}^{p}$ is extensible.

46.6. The isomorphism $\Delta D^{q} E$ maps $\nabla_{\mathbf{A}: K}^{p}$ onto $\delta_{\Gamma: K}^{q-1}$. Let $z^{q-1}$ be an element of $\delta_{\Gamma: K}^{q-1}$. It is known (see for instance [4, p. 246, Theorem III]): Any $\Delta$-cycle on $G_{\alpha 1}^{*}$ is homologous on $G_{\alpha 1}^{*}$ to a cycle of the form $s_{\alpha 1}^{\alpha} z_{\alpha}^{*}$ with $z_{\alpha}^{*}$ on $G_{\alpha}^{*}$. It follows that the $\delta$-class $z^{q-1}$ contains a cycle $s_{\alpha 1}^{\alpha} z_{\alpha}^{*}$, where $z_{\alpha}^{*}$ is a $\Delta$-cycle on $G_{\alpha}^{*}$ and bounds on $K_{\alpha}^{*}$. From 46.1 it follows that there exists a $\nabla$-cycle $z_{\alpha 0}^{p}$ on $A_{\alpha}$ such that $\Delta D_{\alpha}^{q} E_{\alpha} z_{\alpha 0}^{p}$ is homologous to $z_{\alpha}^{*}$ on $G_{\alpha}^{*}$ and therefore

$$
s_{\alpha 1}^{\alpha} \Delta D_{\alpha}^{q} E_{\alpha} z_{\alpha}^{p} \in z^{q-1},
$$

which proves our assertion.

\section{§14. Proof of the combinatorial lemma}

47. The fundamental identity. We write in this paragraph $T$ for nonoriented and $t$ for oriented simplices. The simplex $T_{\alpha 1}^{r}$ of $K_{\alpha 1}$ whose vertices are the centers of the simplices $T_{\alpha}^{n_{0}}>\cdots>T_{\alpha}^{n_{r}}$ will be denoted by

$$
T_{\alpha 1}^{r}=\left|T_{\alpha}^{n_{0}}>\cdots>T_{\alpha}^{n_{r}}\right| \text {. }
$$

A simplex $T_{\alpha 1}^{r}$ of $K_{\alpha 1}$ is called principal if its dimension is the same as the dimension of its carrier in $K_{\alpha}^{*}$; the other simplices of $K_{\alpha 1}$ are called accessory. Among all simplices of $K_{\alpha 1}$ the principal simplices and only these are of the form

$$
T_{\alpha 1}^{q}=\left|T_{\alpha}^{n}>T_{\alpha}^{n-1}>\cdots>T_{\alpha}^{p}\right| .
$$

If we put in correspondence to the center of each simplex $T_{\beta}$ of $K_{\beta}$ the center of the simplex $\sigma_{\alpha}^{\beta} T_{\beta}$ we get a simplicial mapping $\sigma_{\alpha 1}^{\beta 1}$ of $K_{\beta 1}$ into $K_{\alpha 1}$ : for $T_{\beta 1}^{r}=\left|T_{\beta}^{n_{0}}>T_{\beta}^{n_{1}}>\cdots>T_{\beta}^{n_{r}}\right| \in K_{\beta 1}$ we get

$$
{ }_{\sigma_{\alpha 1} 1}^{\beta 1} T_{\beta 1}^{r}=\left|\sigma_{\alpha}^{\beta} T_{\beta}^{n_{0}} \geqq \sigma_{\alpha}^{\beta} T_{\beta}^{n_{1}} \geqq \cdots \geqq \sigma_{\alpha}^{\beta} T_{\beta}^{n_{r}}\right| \in K_{\alpha 1} .
$$

REMARK 1. If $\sigma_{\alpha}^{\beta}$ does not degenerate on $T_{\beta}^{n}$ (that is, if the dimensions of $\sigma_{\alpha}^{\beta} T_{\beta}^{n}$ and $T_{\beta}^{n}$ are the same) then for any simplex $T_{\beta 1}^{q}$ lying on $T_{\beta}^{n}$ the simplex $\sigma_{\alpha 1}^{\beta 1} T_{\beta 1}^{q}$ is the image of $T_{\beta 1}^{q}$ under the affine transformation $\sigma_{\alpha}^{\beta}$ of $T_{\beta}^{n}$.

The manifold $\mathbf{K}$ being orientable, choose any definite orientation of $K_{\alpha}$ and the corresponding orientation of $K_{\beta}$ as well as of all $n$-dimensional simplices $T_{\alpha}^{n}$ and $T_{\beta}^{n}$ of $K_{\alpha}$ and $K_{\beta}$. These orientations will be denoted by $t_{\alpha}^{n}, t_{\beta}^{n}$. For $p<n$ take ad libitum a definite orientation of each $p$-dimensional simplex $T_{\alpha}^{p} \in K_{\alpha}$ and denote this orientation by $t_{\alpha}^{p}$. Choose the orientation $\tau_{\alpha}^{q}$ of the 
corresponding barycentric star $\mathbf{T}_{\alpha}^{q}$ in such a way as to get the intersection number $\left(t_{\alpha}^{p} \times \tau_{\alpha}^{q}\right)=+1$. For $T_{\alpha 1}^{q} \in \mathbf{T}_{\alpha}^{q}$ take the orientation $t_{\alpha 1}^{q}$ corresponding to $\tau_{\alpha}^{q}$.

Choose as follows the orientations $t_{\beta}^{p}$ of the simplices $T_{\beta}^{p}$ of $K_{\beta}$. If $\sigma_{\alpha}^{\beta}$ does not degenerate on $T_{\beta}^{p}$, and $\sigma_{\alpha}^{\beta} T_{\beta}^{p}=T_{\alpha}^{p}$, define $t_{\beta}^{p}$ by the condition $\sigma_{\alpha}^{\beta} t_{\beta}^{p}=t_{\alpha}^{p}$. If $\sigma_{\alpha}^{\beta}$ degenerates on $T_{\beta}^{p}$, choose $t_{\beta}^{p}$ arbitrarily. The orientation $\tau_{\beta}^{q}$ of the barycentric star $\mathbf{T}_{\beta}^{q}$ corresponding to $T_{\beta}^{p}$ is again defined by the condition $\left(t_{\beta}^{p} \times \tau_{\beta}^{q}\right)=+1$. Finally, the orientation $t_{\beta 1}^{q}$ of $T_{\beta 1}^{q} \in \mathbf{T}_{\beta}^{q}$ corresponds to $\tau_{\beta}^{q}$.

The proof of the combinatorial lemma 45.3 rests on the following

FUNDAMENTAL IDENTITY.

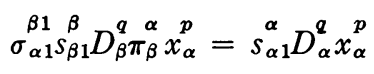

for any chain $x_{\alpha}^{p}$ on $K_{\alpha}$ and any $\beta>\alpha$.

REMARK 2. The mapping $\sigma_{\alpha}^{\beta}$ (of $K_{\beta}$ on $K_{\alpha}$ ), being a natural displacement, has the degree 1 (see $[4$, p. 348 , Theorem II $]$ ). If $\sigma_{\alpha}^{\beta}$ is an arbitrary simplicial mapping of the degree $d$ of the arbitrary $n$-dimensional closed orientable manifold on $K_{\beta}$ on the arbitrary $n$-dimensional closed manifold $K_{\alpha}$, then $(47.1)$ is replaced by

$$
\underset{\sigma_{\alpha 1} s_{\beta 1}^{\beta 1} D_{\beta}^{q \alpha} \pi_{\beta}^{\alpha} x_{\alpha}^{p}}{\beta_{\alpha}}=d s_{\alpha 1}^{\alpha} D_{\alpha}^{q} x_{\alpha}^{p}
$$

for any chain $x_{\alpha}^{p}$ on $K_{\alpha}$ where the operator $\pi_{\beta}^{\alpha}$ is defined as usual by $\left(\pi_{\beta}^{\alpha} x_{\alpha}^{p} \cdot t_{\beta}^{p}\right)$ $=\left(x_{\alpha}^{p} \cdot \sigma_{\alpha}^{\beta} t_{\beta}^{p}\right)$ for any oriented $t_{\beta}^{p} \in K_{\beta}$. In this general form the identity (47.2) formulates a known property of the Hopf "Umkehrhomomorphismus" (see for instance [7]). The proof given below involves this general case.

48. Proof of the fundamental identity. Let us see how the principal simplices are transformed under $\sigma_{\alpha 1}^{\beta 1}$. First of all:

48.1. If the accessory $q$-dimensional simplex

$$
T_{\alpha 1}^{q}=\left|T_{\alpha}^{n_{0}}>\cdots>T_{\alpha}^{n_{q}}\right| \in K_{\alpha 1}
$$

is the image under $\sigma_{\alpha 1}^{\beta 1}$ of the principal simplex $T_{\beta 1}^{q}=\left|T_{\beta}^{n}>T_{\beta}^{n-1}>\cdots>T_{\beta}^{p}\right|$, then $n_{q}<p$.

Proof. From $\sigma_{\alpha}^{\beta} T_{\beta}^{n-i}=T_{\alpha}^{n_{i}}$ follows $n_{i} \leqq n-i$, in particular $n_{0} \leqq n, n_{q} \leqq p$. If $n_{q}=p$, the simplex $T_{\alpha 1}^{q}$ would be principal, and 48.1 is proved.

The principal simplices $T_{\beta 1}^{q}$ mapped under $\sigma_{\alpha 1}^{\beta 1}$ on the principal $T_{\alpha 1}^{q}=\left|T_{\alpha}^{n}>\cdots>T_{\alpha}^{p}\right|$ are easily seen to be in (1-1)-correspondence with the $T_{\beta}^{n} \in K_{\beta}$ mapped under $\sigma_{\alpha}^{\beta}$ on $T_{\alpha}^{n}$. Moreover the orientations $t_{\alpha}^{n}, t_{\beta}^{n}, t_{\alpha}^{p}, t_{\beta}^{p}$, $t_{\alpha 1}^{q}, t_{\beta 1}^{q}$ have been chosen in such a way that from $T_{\beta 1}^{q}=\left|T_{\alpha}^{n}>\cdots>T_{\alpha}^{p}\right|$ and

$$
\begin{aligned}
& { }_{\sigma_{\alpha}}^{\beta} t_{\beta}^{n}=\epsilon t_{\alpha}^{n} \\
& \text { (with } \epsilon= \pm 1 \text { ) }
\end{aligned}
$$

follows 


$$
\stackrel{\beta 1}{\sigma_{\alpha 1} t_{\beta 1}^{q}}=\epsilon t_{\alpha 1}^{q}
$$

with the same $\epsilon$ in (48.11) and (48.12) (the simple proof uses remark 1 of section 47). Therefore for any principal simplex $t_{\alpha 1}^{q}$

$$
\left(\sigma_{\alpha 1}^{\beta 1} \sum t_{\beta 1}^{q} \cdot t_{\alpha 1}^{q}\right)=\left(\sigma_{\alpha}^{\beta} \sum t_{\beta}^{n} \cdot t_{\alpha}^{n}\right),
$$

where the sum is taken over all principal $t_{\beta 1}^{q}$ at the left and over all $n$-dimensional simplices of $K_{\beta}$ at the right. Since the value of $\sigma_{\alpha}^{\beta} \sum t_{\beta}^{n}$ on $t_{\alpha}^{n}$ is equal to the degree $d$ we get

$$
\sigma_{\alpha 1}^{\beta 1} \sum t_{\beta 1}^{q}=d \sum t_{\alpha 1}^{q}
$$

(summation on both sides over all $q$-dimensional principal simplices of $K_{\beta 1}$ and $K_{\alpha 1}$ respectively).

The proof of the fundamental identity presents no more difficulty. To simplify the notations put

$$
\stackrel{q}{y_{\beta 1}}=\stackrel{\beta}{s_{\beta 1}} D_{\beta}^{q \alpha} \pi_{\beta}^{\alpha} x_{\alpha}^{p} .
$$

We shall calculate the value of the chain $y_{\beta 1}^{q}$ on an arbitrary simplex $t_{\beta 1}^{q}$ of $K_{\beta 1}$. If $t_{\beta 1}^{q}$ is accessory this value is zero by the very definition of the operator $s_{\beta 1}^{\beta}$. If $t_{\beta 1}^{q}$ is a principal simplex, let $\tau_{\beta}^{q}$ be its carrier in $K_{\beta}^{*}$ and $t_{\beta}^{p}$ the corresponding (oriented) simplex of $K_{\beta}$. By the definition of $s_{\beta 1}^{\beta}, D_{\beta}^{q}$ and $\pi_{\beta}^{\alpha}$ we have

$$
\left(y_{\beta 1}^{q} \cdot t_{\beta 1}^{q}\right)=\left(x_{\alpha}^{p} \cdot \sigma_{\alpha}^{\beta} t_{\beta}^{p}\right) \text {. }
$$

To evaluate the left-hand side of the identity (47.2) take any $T_{\alpha 1}^{q} \in K_{\alpha 1}$ and all principal simplices

$$
T_{j}^{q}=\left|T_{\beta}^{n}(j)>\cdots>T_{\beta}^{p}(j)\right| \in K_{\beta 1}, \quad j=1,2, \cdots, \nu,
$$

which are mapped by $\sigma_{\alpha 1}^{\beta 1}$ on $T_{\alpha 1}^{q}$. Let

$$
\sigma_{\alpha 1}^{\beta 1} t_{j}^{q}=\epsilon_{j} t_{\alpha 1}^{q}, \quad \quad \epsilon_{j}= \pm 1 .
$$

We have by (48.3) and the definition of the $\epsilon_{j}$ :

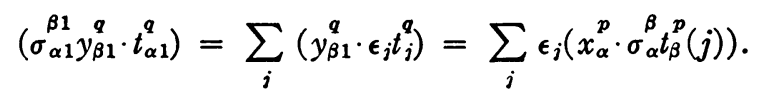

If $T_{\alpha 1}^{Q}$ is accessory then by (48.1) we have $n_{q}<p$; it follows-since $\sigma_{\alpha}^{\beta} T_{\beta}^{p}(j)=T_{\alpha}^{n_{q}}$ - that $\left(x_{\alpha}^{p} \cdot \sigma_{\alpha}^{\beta} t_{\beta}^{p}(j)\right)=0$, therefore by $(48.4)$

$$
\left(\sigma_{\alpha 1}^{\beta 1} y_{\beta 1}^{q} \cdot t_{\alpha 1}^{q}\right)=0 .
$$

Obviously $\left(s_{\alpha 1}^{\alpha} D_{\alpha}^{q} x_{\alpha}^{p} \cdot t_{\alpha 1}^{q}\right)=0$, thus both sides of (47.2) take on the accessory $t_{\alpha 1}^{q}$ the value zero.

If $T_{\alpha 1}^{q}$ is principal, $T_{\alpha 1}^{q}=\left|T_{\alpha}^{n}>\cdots>T_{\alpha}^{p}\right|$, then $\sigma_{\alpha}^{\beta} T_{\beta}^{p}(j)=T_{\alpha}^{p}$, and even $\sigma_{\alpha}^{\beta} t_{\beta}^{p}(j)=t_{\alpha}^{p}$, hence by $(48.4)$ 


$$
\left(\sigma_{\alpha 1}^{\beta 1} y_{\beta 1}^{q} \cdot t_{\alpha 1}^{q}\right)=\sum_{j} \epsilon_{j}\left(x_{\alpha}^{p} \cdot t_{\alpha}^{p}\right)=\left(x^{p} \cdot t_{\alpha}^{p}\right) \sum_{j} \epsilon_{j} .
$$

By (48.2) $\sum_{j} \epsilon_{j}=d$ and therefore

$$
\left(\begin{array}{c}
\beta 1 \\
\sigma_{\alpha 1} y_{\beta 1}^{q} \cdot t_{\alpha 1}^{q}
\end{array}\right)=d\left(x_{\alpha}^{p} \cdot t_{\alpha}^{p}\right) .
$$

So much for the left-hand side of (47.2). At the right, the chain $s_{\alpha 1}^{\alpha} D_{\alpha}^{a} x_{\alpha}^{p}$ takes the value $\left(x_{\alpha}^{p} \cdot t_{\alpha}^{p}\right)$ on $t_{\alpha 1}^{q}$, which completes the proof of the identity (47.2).

49. The rest of the proof of Lemma (45.3). This is based on the following two elementary propositions:

49.1. Let $\Gamma_{\alpha}, \Gamma_{\alpha 1}^{*}$ denote the set theoretical sum of the simplices of $G_{\alpha}, G_{\alpha 1}^{*}$ respectively. There exists a continuous mapping $C$ of the point set $\Gamma_{\alpha}$ onto the point set $\Gamma_{\alpha 1}^{*}$ leaving all points of $\Gamma_{\alpha 1}^{*}$ invariant.

49.2. Let the $\nabla$-cycle $z_{\alpha}^{p}$ on $K_{\alpha}$ be equal to zero on $A_{\alpha}$; if $z_{\beta 1}^{q}=s_{\beta 1}^{\beta} D_{\beta}^{q} \pi_{\beta}^{\alpha} z_{\alpha}^{p}$ differs from zero on a given $T_{\beta 1}^{q} \in K_{\beta 1}$ then a convex subset of $\Gamma_{\alpha}$ contains both the closures of $T_{\beta 1}^{q}$ and of $\sigma_{\alpha 1}^{\beta 1} T_{\beta 1}^{q}$.

Proof of 49.1. The open set $\Gamma_{\alpha}$ is the set theoretical sum of the simplices $T_{\alpha 1} \in K_{\alpha 1}$ lying on it. Each of the simplices $T_{\alpha 1} \in K_{\alpha 1}$ lying on $\Gamma_{\alpha}$ but not belonging to $G_{\alpha 1}^{*}$ is of the form

$$
T_{\alpha 1}^{r}=\left|T_{\alpha}^{n_{0}}>\cdots>T_{\alpha}^{n_{i}}>\cdots>T_{\alpha}^{n_{r}}\right|,
$$

where $T_{\alpha}^{n_{0}}, \ldots, T_{\alpha}^{n_{i}}$ belong to $G_{\alpha}$, while $T_{\alpha}^{n_{i+1}}, \cdots, T_{\alpha}^{n_{r}}$ are elements of $A_{\alpha}$. Thus each point $a$ of $T_{\alpha 1}^{r}$ is on a segment $\left|a^{\prime} a^{\prime \prime}\right| \subseteq T_{\alpha 1}^{r}$ joining a point $a^{\prime}$ of the simplex $T_{\alpha 1}^{\prime}=\left|T_{\alpha}^{n_{0}}>\cdots>T_{\alpha}^{n_{i}}\right| \in G_{\alpha 1}^{*}$ with a point $a^{\prime \prime}$ of $T_{\alpha 1}=\left|T_{\alpha}^{n_{i+1}}>\cdots>T_{\alpha}^{n_{r}}\right|$, the simplex $T_{\alpha 1}^{\prime \prime}$ belonging to the barycentric subdivision $A_{\alpha 1}$ of $A_{\alpha}$. If we put $C(a)=a^{\prime}$ for $a \in \Gamma_{\alpha} \backslash \Gamma_{\alpha 1}^{*}$ and $C(a)=a$ for $a \in \Gamma_{\alpha 1}^{*}$ we obtain a continuous mapping $C$ with the required properties.

Proof of 49.2. Let $T^{n}$ be a simplex, $T^{p}$ a face of $T^{n}$. Denote by $S\left(T^{p}, T^{n}\right)$ the complex composed of $T^{n}$ and all faces $T^{r}$ of $T^{n}$ satisfying the condition $T^{r} \geqq T^{p}$ (where $T^{\prime}>T^{\prime \prime}$ means that the simplex $T^{\prime \prime}$ is a face of $T^{\prime}$ ). Denote by $\sum\left(T^{p}, T^{n}\right)$ the set theoretical sum of all elements of $S\left(T^{p}, T^{n}\right)$. Among all points of the closed set $\bar{T}^{n}$ the points of $\sum\left(T^{p}, T^{n}\right)$ are characterized by the property that their barycentric coordinates (in the coordinate system defined by the vertices of $T^{n}$ ) corresponding to the vertices of $T^{p}$ are all positive. Therefore the point set $\sum\left(T^{p}, T^{n}\right)$ is convex. This obvious remark will be applied now to the proof of 49.2 . The hypotheses of 49.2 imply that $T_{\beta 1}^{q}$ is a principal simplex,

$$
T_{\beta 1}^{q}=\left|T_{\beta}^{n}>\cdots>T_{\beta}^{p}\right|,
$$

and that $\pi_{\beta}^{\alpha} z_{\alpha}^{p}$ differs from zero on $T_{\beta}^{p}$; hence

$$
{ }_{\sigma_{\alpha}}^{\beta} T_{\beta}^{p}=T_{\alpha}^{p} \in G_{\alpha} .
$$

Denote by $T_{\alpha}^{n}$ the carrier of $T_{\beta}^{n}$ in $K_{\alpha}$, and put 


$$
T_{\alpha}^{n_{0}}=\sigma_{\alpha}^{\beta} T_{\beta}^{n}
$$

The mapping $\sigma_{\alpha}^{\beta}$ being a natural displacement, we have

$$
T_{\alpha}^{n_{0}} \leqq T_{\alpha}^{n}
$$

and therefore

$$
T_{\alpha}^{p}=\sigma_{\alpha}^{\beta} T_{\beta}^{p} \leqq T_{\alpha}^{n} .
$$

Since $T_{\alpha}^{p} \in G_{\alpha}$ and $G_{\alpha}$ is open in $K_{\alpha}$, we have

$$
S\left(T_{\alpha}^{p}, T_{\alpha}^{n_{0}}\right) \subseteq S\left(T_{\alpha}^{p}, T_{\alpha}^{n}\right) \subseteq G_{\alpha} .
$$

The vertices of $\sigma_{\alpha 1}^{\beta 1} T_{\beta 1}^{q}$, being the centers of the simplices

$$
T_{\alpha}^{n_{0}}={ }_{\sigma_{\alpha}}^{\beta} T_{\beta}^{n} \geqq \dot{\sigma}_{\alpha}^{\beta} T_{\beta}^{n-1} \geqq \cdots \geqq \sigma_{\alpha}^{\beta} T_{\beta}^{p}=T_{\alpha}^{p},
$$

are points of the set $\sum\left(T_{\alpha}^{p}, T_{\alpha}^{n_{0}}\right) \subseteq \sum\left(T_{\alpha}^{p}, T_{\alpha}^{n}\right)$. Since the point set $\sum\left(T_{\alpha}^{p}, T_{\alpha}^{n}\right)$ is convex and contains the vertices of the simplex $\sigma_{\alpha 1}^{\beta 1} T_{\beta 1}^{q}$ it contains the closure of this simplex. It remains to prove that the closure of the simplex $T_{\beta 1}^{\alpha}$ is contained in the same convex set $\sum\left(T_{\alpha}^{p}, T_{\alpha}^{n}\right)$. Let $T_{\alpha}^{n_{i}}, i=1,2, \cdots, q$, be the carrier of $T_{\beta}^{n-1}$ in $K_{\alpha}$. Since $T_{\alpha}^{n}$ is the carrier of $T_{\beta}^{n}$ and $T_{\alpha}^{p}=\sigma_{\alpha}^{\beta} T_{\beta}^{p} \leqq T_{\alpha}^{n_{\alpha}}$, we have

$$
T_{\alpha}^{n} \geqq T_{\alpha}^{n_{1}} \geqq \cdots \geqq T_{\alpha}^{n_{q}} \geqq T_{\alpha}^{p},
$$

whence it follows that $T_{\alpha}^{n}, T_{\alpha}^{n_{1}}, \cdots, T_{\alpha}^{n_{q}}$ and therefore the centers of $T_{\beta}^{n} \subseteq T_{\alpha}^{n}$, $T_{\beta}^{n-1} \subseteq T_{\alpha}^{n_{1}}, \cdots, T_{\beta}^{p} \subseteq T_{\alpha}^{n_{q}}$ are on $\sum\left(T_{\alpha}^{p}, T_{\alpha}^{n}\right)$; since $\sum\left(T_{\alpha}^{p}, T_{\alpha}^{n}\right)$ is convex, the closure of the simplex $T_{\beta 1}^{q}=\left|T_{\beta}^{n}>T_{\beta}^{n-1}>\cdots>T_{\beta}^{p}\right|$ is a subset of $\sum\left(T_{\alpha}^{p}, T_{\alpha}^{n}\right)$ and 49.2 is proved.

It follows from 49.2 that the prism with the bases $z_{\beta 1}^{q}$ and $\sigma_{\alpha 1}^{\beta 1} z_{\beta 1}^{q}$ (see section 28) lies on a polyhedron $\Pi \subseteq \Gamma_{\alpha}$ (composed of the simplices of this prism) and thus $z_{\beta 1}^{\alpha} \sim \sigma_{\alpha 1}^{\beta 1} z_{\beta 1}^{q}$ on $\Pi \subseteq \Gamma_{\alpha}$ (this homology being understood in some invariant sense-either as a continuous homology in the sense of $[4, \mathrm{p} .335]$ or as a homology between convergent cycles [5, pp. 240, 241]). If we take $z_{\beta i}^{2}$ from (49.2). we obtain

$$
\stackrel{s_{\beta 1}}{\beta} D_{\beta}^{q \alpha \pi_{\beta} z_{\alpha}^{p}} \sim \sigma_{\alpha 1}^{\beta 1} s_{\beta 1}^{\beta} D_{\beta}^{q} \pi_{\beta}^{\alpha} z_{\alpha}^{p} \text { on } \Pi \subseteq \Gamma_{\alpha} \subseteq \Gamma_{\beta}
$$

On the other hand, we have by (47.1)

$$
\stackrel{\alpha}{s_{\alpha 1} D_{\alpha}^{q} z_{\alpha}^{p}}=\underset{\sigma_{\alpha 1} s_{\beta 1}{ }^{\beta}}{q} D_{\beta}^{q} \pi_{\beta}^{\alpha} z_{\alpha}^{p}
$$

thus $s_{\alpha 1}^{\alpha} D_{\alpha}^{\alpha} z_{\alpha}^{p}$ (considered as a continuous or as a convergent cycle) lies on $\Pi$ and therefore

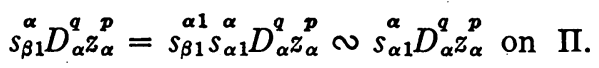

Hence we may replace in (49.3) the chain $\sigma_{\alpha 1}^{\beta 1} s_{\beta 1}^{\beta} D_{\beta}^{q} \pi_{\beta}^{\alpha} z_{\alpha}^{p}$ by $s_{\beta 1}^{\alpha} D_{\alpha}^{q} z_{\alpha}^{p}$, which gives 


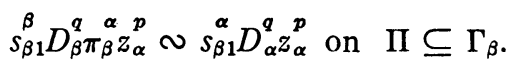

By the mapping $C$ of 49.1 this homology turns into

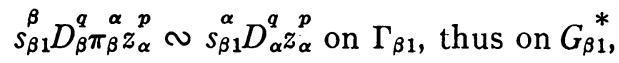

which completes the proof of the combinatorial lemma, as well as the proof of the general duality theorem of Alexander-Pontrjagin.

\section{\$15. THE REMOVING THEOREM}

50. The removing theorem in the case of a manifold. A $\nabla$-cycle $z_{\alpha}^{p}$ on $K_{\alpha}$ is homologous on $K_{\alpha}$ to a cycle on $G_{\alpha}$ if and only if the $\Delta$-cycle $D_{\alpha}^{q} z_{\alpha}^{p}$ is homologous on $K_{\alpha}^{*}$ to a cycle on $G_{\alpha}{ }^{*}$. As the scalar product of $z_{\alpha}^{p}$ with a $\Delta$-cycle $z_{\alpha 0}^{p}$ on $A_{\alpha}$ is equal to the intersection number of $D_{\alpha}^{q} z_{\alpha}^{p}$ with the same $z_{\alpha 0}^{p}$ we get the following form of

The REMOVING THEOREM FOR MANIFOLDS. $A \delta$-class $z^{q}$ on the ( $n$-dimensional orientable) manifold $\mathbf{K}$ contains as a subset a $\delta$-class on the given open set $\boldsymbol{\Gamma}=\mathbf{K} \backslash \mathbf{A}$ if and only if, whatever be the thread $z_{0}^{p}=\left\{z_{\alpha \alpha 0}^{p}\right\} \in \Delta_{\alpha}^{p}$ and $z_{\alpha}^{q} \in z^{q}$, the intersection of $z_{\alpha}^{q}$ with any $z_{\alpha 0}^{p} \in_{\zeta \alpha 0}^{p}$ is equal to zero (here, as usual, the $\delta$-classes are taken with respect to a discrete coefficient-group $X$, while the coefficient-group in $\Delta_{\alpha}^{p}$ is $\left.\Xi \mid X\right)$.

Another formulation of the same theorem is:

A $q$-dimensional convergent cycle $z^{q}$ (with respect to the discrete group $X$ ). on $\mathbf{K}$ is homologous on $\mathbf{K}$ to a cycle on $\boldsymbol{\Gamma}$ if and only if the intersection of $z^{q}$ with each $p$-dimensional convergent cycle on $\mathbf{A}$ (with respect to $\Xi \mid X$ ) is equal to zero.

REMARK 1. The theorem holds also for $X=\Xi=\Re$ (see section 5).

REMARK 2. For non-orientable manifolds the theorem holds in the special case $X=\Xi=I_{2}$.

\section{BiBLIOGRAPHY}

1. P. Alexandroff, General combinatorial topology, Trans. Amer. Math. Soc. vol. 49 (1941) pp. 41-105.

2. - (a) Homologiegruppen allgemeiner Projectionsspectra; (b) Allgemeiner Dualitätssatz für Projectionsspectra und für im Kleinen bikompakte Räume, Mitteilungen der Georgischen Abteilung der Akademie der Wissenschaften der SSR vol. 2 (1941) pp. 213-219 and 315-319 (in Russian with a German summary).

3. - Zurïckfürung des Alexander-Pontrjaginschen Dualitätssatzes auf den Dualitätssatz von Kolmogoroff, ibid. vol. 1 (1940) pp. 401-410.

4. P. Alexandroff and H. Hopf, Topologie, I, 1935.

5. P. Alexandroff, H. Hopf, L. Pontrjagin, Über den Brouwerschen Dimensionsbegriff, Compositio Math. vol. 4 (1935) pp. 239-255.

6. M. Bockstein, Über die Homologiegruppen der Vereinigung zweier Komplexe, Rec. Math. (Mat. Sbornik) N.S. vol. 9 (1941) pp. 365-376. 
7. H. Freudenthal, Zum Hopfschen Umkehrhomomorphismus, Ann. of Math. (2) vol. 38 (1937) pp. 847-853.

8. A. Kolmogoroff, Über die Dualität im Aufbau der kombinatorischen Topologie, Rec. Math. (Mat. Sbornik) N.S. vol. 1 (1936) pp. 97-102.

9. S. Lefschetz, On closed sets on a manifold, Ann. of Math. (2) vol. 29 (1928) pp. 232-254.

10. L. Pontrjagin, Topological groups.

11. - Zum Alexanderschen Dualitätssatz. II, Nachr. Ges. Wiss. Göttingen, 1927, pp. 446-454.

12. Seifert-Threlfall, Topologie, 1934.

13. N. E. Steenrod, On universal homology groups, Amer. J. Math. vol. 48 (19.36) pp. $661-$ 701.

14. A. W. Tucker, An abstract approach to manifolds, Ann. of Math. (2) vol. 34 (1935) pp. 191-243.

Mathematical Institute of the Academy of Sciences,

KASAN, USSR, January-June, 1942 